\title{
Improvements in the Measurement of Creep Crack Initiation and Growth Using Potential Drop
}

\author{
K. M. Tarnowski ${ }^{1}$, K. M. Nikbin ${ }^{1}$, D. W. Dean ${ }^{2}$ and C. M. Davies ${ }^{1}$ \\ ${ }^{1}$ Department of Mechanical Engineering, Imperial College London, South Kensington \\ Campus, London, SW7 2AZ. UK. \\ ${ }^{2}$ EDF Energy, Barnett Way, Barnwood, Gloucester, GL4 3RS. UK.
}

\begin{abstract}
:
To predict the residual life of components operating in the creep regime, it is vital to accurately identify crack initiation, and measure subsequent crack growth, in laboratory tests. Potential drop (PD) measurements, used for this purpose, are susceptible to errors caused by the accumulation of creep strain. For creep ductile materials, this can result in highly conservative crack initiation models and the implementation of unnecessary inspection and maintenance programmes that can cost millions of pounds in lost revenue. Conversely, the crack growth models can be non-conservative.

Using a novel combination of interrupted creep crack growth (CCG) tests and sequentially coupled structural-electrical finite element analyses a new method of interpreting PD data has been developed and validated. It uses an increase in gradient on a plot of PD vs. load-line displacement to accurately identify crack initiation. This has been compared to the current method in ASTM E1457-15 by reanalysing data from CCG tests performed on a range of materials at various temperatures and loads. The initiation times, measured using the current ASTM method, were underestimated by factors of up to 23 and the subsequent crack growth rates were underestimated by factors of up to 1.5 .
\end{abstract}

Keywords: Creep crack initiation; Creep crack growth; Potential drop method; Strain; Finite element analysis 


\section{Nomenclature}

\section{$1.1 \quad \underline{\text { Symbols }}$}

$\begin{array}{ll}a & \text { Crack length } \\ a_{0} & \text { Initial crack length } \\ a_{f} & \text { Final crack length measured from the post-test fracture surface } \\ a_{p} & \text { Predicted crack length from the potential drop technique } \\ a_{p f} & \text { Predicted final crack length from the potential drop technique } \\ B & \text { Specimen thickness } \\ B_{N} & \text { Net specimen thickness } \\ C, C_{1}, C_{2}, C_{3} & \text { Creep law material constants } \\ C^{*} & \text { Crack tip characterising parameter } \\ E & \text { Young's modulus } \\ n, n_{1}, n_{3} & \text { Creep law material constants } \\ t & \text { Time }\end{array}$

$t_{0}^{A S T M} \quad$ Time to the onset of crack growth as determined in ASTM E1457-15

$t_{0}^{M O D} \quad$ Time to the onset of crack growth as determined by the proposed modified method

$t_{0.2}^{A S T M} \quad$ Time for $0.2 \mathrm{~mm}$ of crack growth as determined in ASTM E1457-15

$t_{0.2}^{M O D} \quad$ Time for $0.2 \mathrm{~mm}$ of crack growth as determined by the proposed modified method

$t_{f p} \quad$ Time corresponding to the transition from primary to secondary creep

$R \quad$ Resistance

$R_{0} \quad$ Normalising resistance

$R_{0}^{A S T M} \quad$ Normalising resistance as determined in ASTM E1457-15

$R_{0}^{M O D} \quad$ Normalising resistance as determined in the proposed modified method

V Potential drop

$V_{0} \quad$ Normalising potential drop

$V_{0}^{A S T M} \quad$ Normalising potential drop as determined in ASTM E1457-15

$V_{0}^{M O D} \quad$ Normalising potential drop as determined in the proposed modified method

W Specimen width

$v \quad$ Poisson's ratio 


\subsection{Acronyms}

$\mathrm{AC}$

Alternating Current

ACPD

Alternating Current Potential Drop

$\mathrm{C}(\mathrm{T})$

Compact Tension

DC

Direct Current

DCPD

Direct Current Potential Drop

EDM

Electrical Discharge Machined

FE

Finite Element

CCG

Creep Crack Growth

LLD

Load-Line Displacement

PD

Potential Drop

\section{Introduction}

The justification for continued safe operation of structures operating in the creep regime relies upon accurate material models. Overly conservative material models can prompt expensive and unnecessary inspection and maintenance programmes, e.g. the lost revenue associated with the shutdown of a UK Advanced Gas-Cooled Reactor (AGR) is $\sim £ 0.5 \mathrm{~m} /$ day (based on a typical wholesale electricity price of £40/MWh (Ofgem, 2016), and a typical reactor capacity of 500MW (Houlton, 2013)). Conversely, material models which are non-conservative are potentially dangerous. To develop accurate material models it is vital to be able to precisely measure material behaviour in the laboratory.

The life of a structure operating in the creep regime is often limited by the initiation and growth behaviour of pre-existing defects such as those contained in welds. To develop material models which capture this behaviour it is necessary to be able to accurately identify crack initiation, and measure subsequent crack growth, in the laboratory. The Potential Drop (PD) technique is almost exclusively used to perform these measurements because the hostile environment and static load requirements prohibit most alternative methods (Wilkowski and Maxey, 1983).

It is well known that strain influences the electrical behaviour of metals. It is this phenomenon which is utilised in a typical resistance strain gauge. It is therefore not surprising that strain has been identified as a significant source of error when using the PD technique to measure crack initiation and growth in ductile materials (Freeman and Neate, 1980; Saxena, 1980). The influence of strain on PD measurements tends to be most significant prior to crack initiation (the onset of crack growth) (Bakker, 1985; Freeman and Neate, 1980; Ljustell, 2011; Lowes and Fearnehough, 1971). Under creep conditions this is often known as the incubation period 
and can account for the majority of the life of a component (Haigh, 1975; Merah et al., 1995). It has also been suggested that, in some cases, strain may continue to significantly influence the PD response during crack growth (Lowes and Fearnehough, 1971; Saxena, 1980).

When testing creep ductile materials, strain can cause the PD technique to underestimate the incubation period (Webster and Ainsworth, 1994) and overestimate the extent of subsequent crack growth (Saxena, 1980) such that the resulting material models will produce overly conservative predictions of the residual life of real structures. To improve the accuracy of these models it is necessary to separate the change in PD due to crack growth from that due to strain. This non-trivial problem also applies when using the PD technique to measure stable tearing during fracture toughness testing of ductile materials. Lowes and Fearnehough (Lowes and Fearnehough, 1971) proposed an experimental method of achieving this which has since been incorporated into some fracture toughness testing standards, e.g. ESIS P2-92 and ISO 121352002.

\subsection{Measuring Crack Initiation and Growth during Fracture Toughness Testing}

The method proposed by Lowes and Fearnehough (Lowes and Fearnehough, 1971) is shown schematically

in

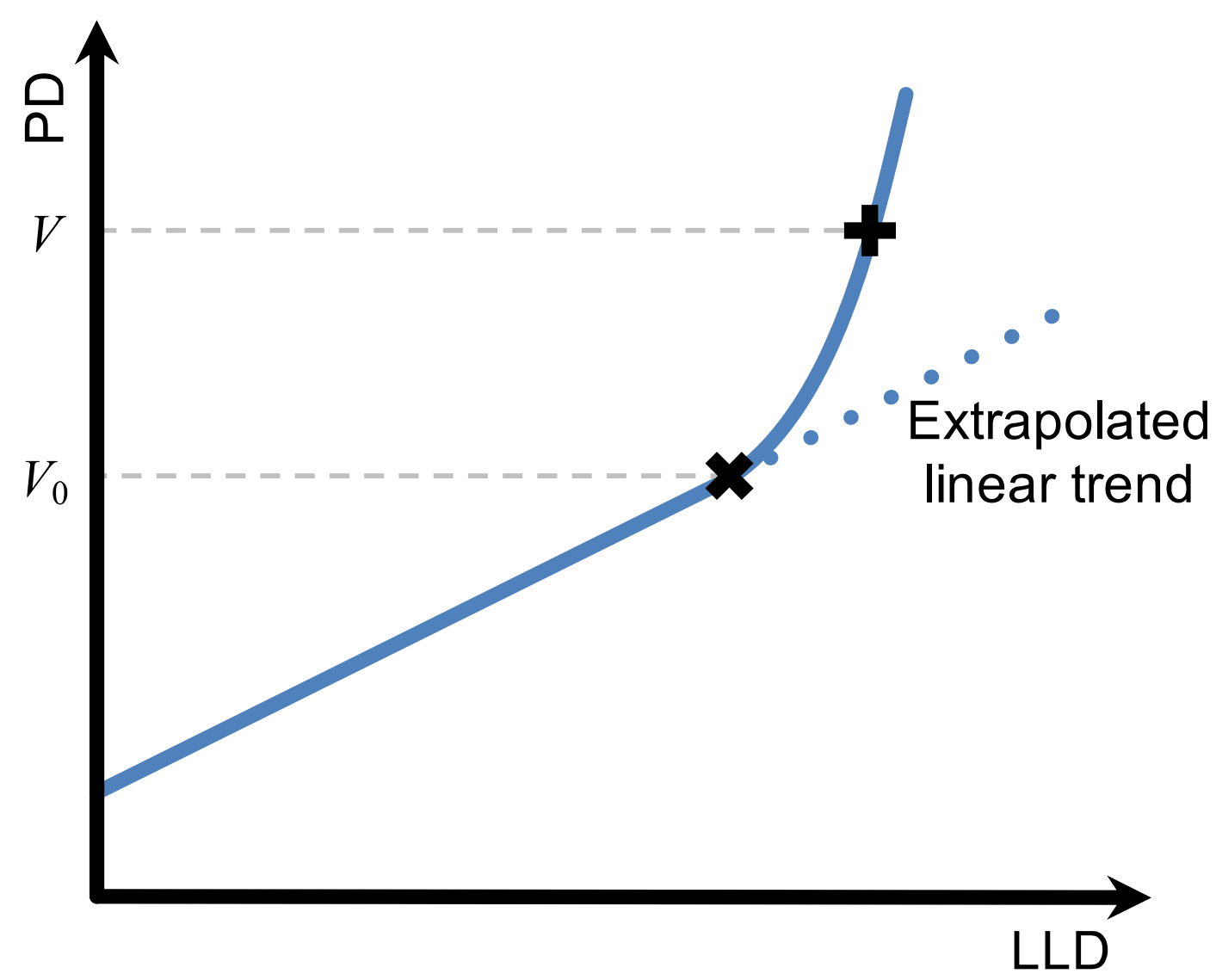


Figure 1. A linear relationship between PD and Load-Line Displacement (LLD) is typically observed prior to the onset of stable tearing. When the crack initiates the experimental data deviates from this linear trend. The PD at this point, labelled ' $x$ ' in

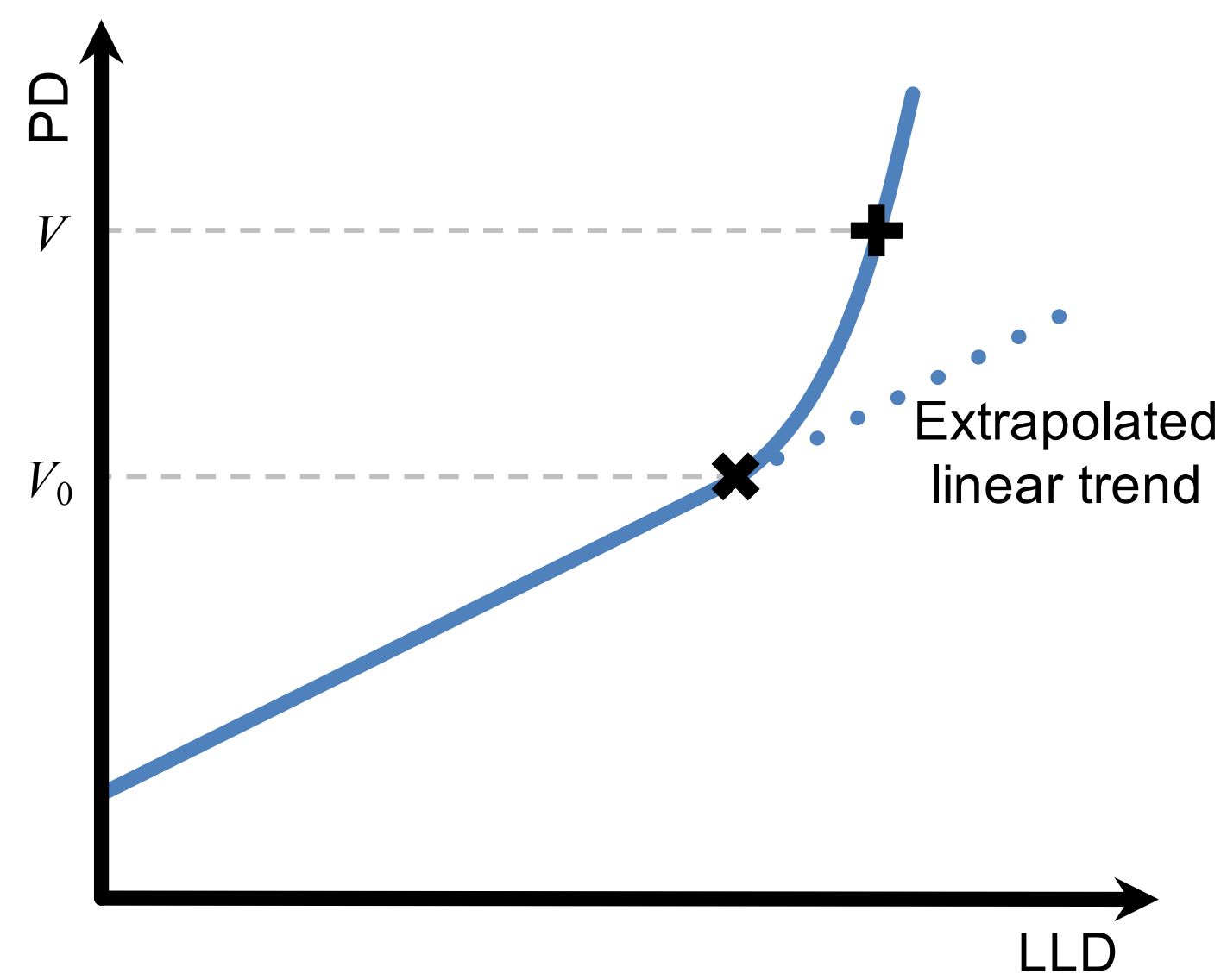

Figure 1 , is denoted by $V_{0}$. At some arbitrary point later in the test, labelled ' + ', the corresponding PD value is denoted $V$, and the crack extension can be estimated using a calibration function and the values $V_{0}$ and $V$.

An example third order polynomial calibration function is provided in Equation (1) where $a_{p}$ is the predicted crack length. Calibration functions are typically derived assuming that the PD 
is only influenced by crack growth. The method shown in

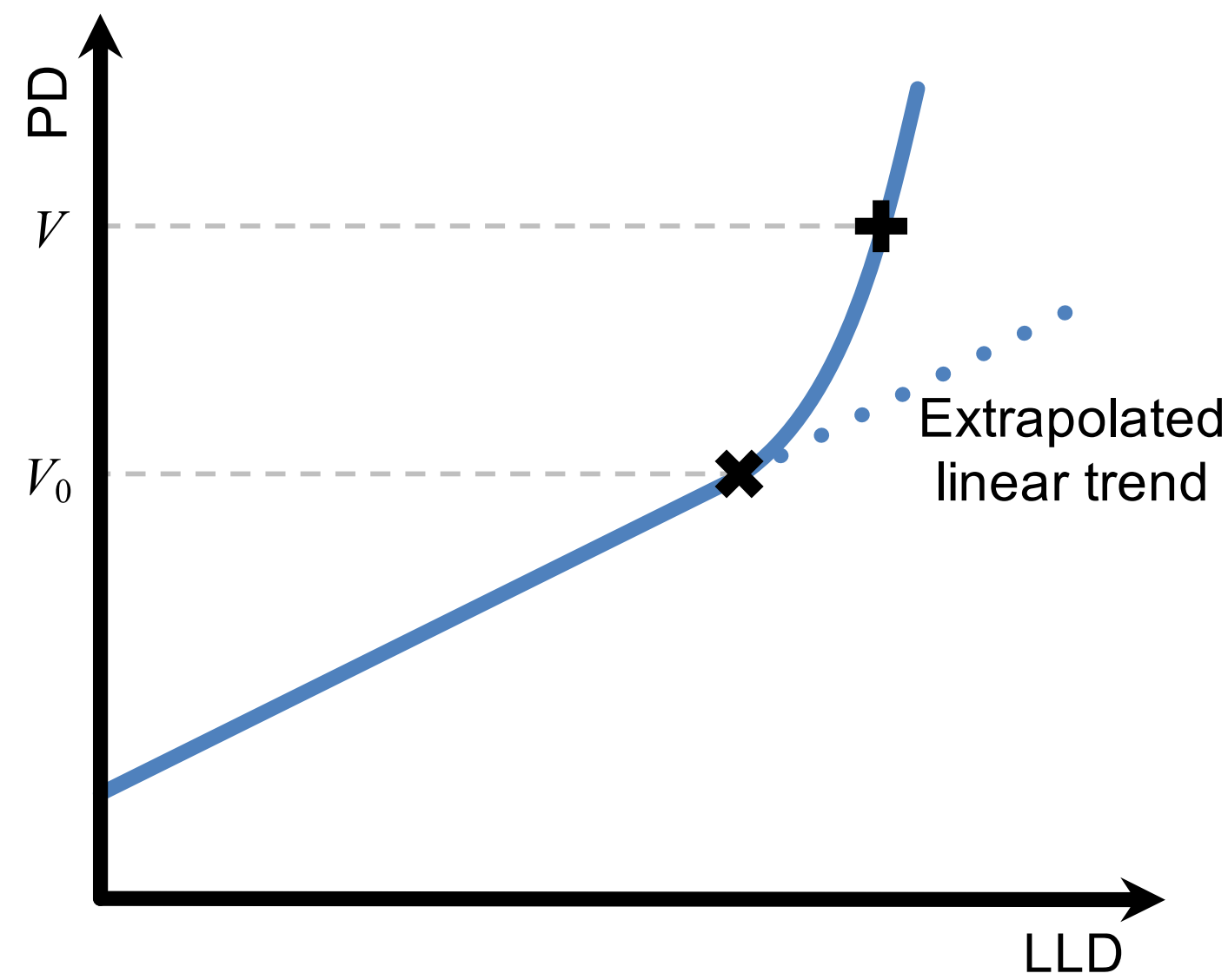

Figure 1 therefore assumes that the influence of strain is negligible after the onset of stable tearing.

$$
\frac{a_{p}}{W}=A_{0}+A_{1}\left(\frac{V}{V_{0}}\right)+A_{2}\left(\frac{V}{V_{0}}\right)^{2}+A_{3}\left(\frac{V}{V_{0}}\right)^{3}
$$

During fracture toughness testing it can be difficult to identify the exact point at which the crack initiates. To mitigate this, an 'engineering definition' of initiation is often applied. This corresponds to $0.2 \mathrm{~mm}$ of crack extension, which is a small but measurable amount of crack extension e.g. ESIS P2-92 and ISO 12135:2002.

\subsection{Measuring Creep Crack Initiation and Growth}

An equivalent approach to mitigate the effects of strain is currently not available for Creep Crack Growth (CCG) testing. When interpreting test data in accordance with ASTM E1457-15 (the most common CCG testing standard) any increase in PD, after the load is applied, is attributed to crack growth. This assumes that the influence of creep strain is negligible. As discussed above, experimental data interpreted in this way will result in overly conservative 
predictions of the residual life of real structures. Consistent with fracture toughness testing, ASTM E1457-15 incorporates an 'engineering definition' of initiation, corresponding to $0.2 \mathrm{~mm}$ of crack extension.

During CCG testing the final crack size predicted using the PD technique, $a_{p f}$, often does not agree with optical measurements from the post-test fracture surface, $a_{f}$. As well as the influence of strain, this can be due to differences between the ideal crack geometry assumed in the derivation of the calibration function and the actual crack geometry obtained experimentally. Typical differences include non-uniform crack extension along the crack front, electrical shorting across the crack faces associated with asperities on the fracture surface and discontinuous cracking. These differences are often cited as sources of error in the measurement of crack extension (Fessler et al., 2017; McIntyre and Priest, 1971; Merah, 2003; Riemelmoser et al., 1999). To mitigate these errors the predicted crack length may be corrected by linear interpolation using Equation (2) where $a_{0}$ is the initial crack length at the start of the test. Guidance in ASTM E1457-15 suggests that this correction may only be applied to cases where the crack extension predicted by the PD technique is within $15 \%$ of the value measured from the post-test fracture surface.

$$
a=\left[\frac{\left(a_{f}-a_{0}\right)}{\left(a_{p f}-a_{0}\right)}\left(a_{p}-a_{0}\right)\right]+a_{0}
$$

During CCG testing an initial drop in PD is sometimes observed. The cause of this behaviour remains unknown although it has been suggested that it may be related to dislocation rearrangement during primary creep and changes in precipitate size and spacing (Freeman and Neate, 1980). To mitigate this, the guidance in ASTM E1457-15 is to extrapolate the minimum value of PD back to zero time, i.e. the minimum PD value is taken as the value of $V_{0}$ used in the calibration function.

\subsection{The Influence of Strain on PD Measurements}

Experimental methods of separating the change in PD due to strain from the change in PD due to crack growth have historically been validated using interrupted tests, e.g. (Lowes and Fearnehough, 1971). This allows observations from the post-test fracture surface to be compared with the PD response however, this process is slow and laborious and it is difficult to control other variables which may also influence the PD response such as crack morphology. 
An alternative approach has been developed which uses a sequentially coupled structural-electrical Finite Element (FE) model to predict the influence of strain on PD measurements. Such models only capture the geometric effects of strain (the change in resistance associated with deformation) and not the material effects (the change in resistivity) but for inelastic strains, which are driven by dislocations, these material effects are small (Ke and Ståhle, 1993). This FE based approach has been used to predict the PD response due to strain for monotonically loaded stationary cracks (Ke and Ståhle, 1993; Ljustell, 2011). Recently it has also been extended to include crack growth and has been used to confirm that the onset of stable tearing during fracture toughness testing coincides with an increase in gradient on a plot of PD vs. LLD (Tarnowski et al., 2017).

There is a close analogy between creep and plasticity which is often exploited to interpret creep behaviour. Continuing this analogy, the aim of the present study assess if an increase in gradient on a plot of PD vs. LLD can be used to identify initiation during CCG tests, similar to the approach proposed by Lowes and Fearnehough for fracture toughness tests. This novel approach to interpreting PD measurements would supress any change in PD due to creep strain which occurs prior to initiation, removing potentially excessive conservatism from the measurement of the incubation period. Such measurements would permit the justification for continued safe operation of real structures providing potentially significant cost savings.

In this study a combination of approaches has been employed. Interrupted CCG tests have been used to compare the crack extension predicted from the PD response with observations from the post-test fracture surface. These experimental observations have been validated using a novel sequentially coupled structural-electrical FE model of a CCG test. This is the first time creep deformation has been incorporated into such models. Finally, experimental data from CCG tests performed on a range of materials, temperatures and test durations has been reanalysed to confirm that a similar response is observed for a wide range of test scenarios.

\section{Experimental Testing}

A preliminary CCG test was performed on ferritic P91 steel at $620^{\circ} \mathrm{C}$ to investigate the influence of creep strains on PD measurements in the absence of crack extension. This is a material with a particularly high creep ductility ( 32\% (NIMS, 2014)) so influence of creep strains should be significant. This was followed by a series of interrupted tests, terminated at various points before and after initiation to investigate the PD response throughout the test. These tests were performed on ex-service austenitic Type $316 \mathrm{H}$ stainless steel (cast 55882) at 
$550{ }^{\circ} \mathrm{C}$. This is another creep ductile material although the creep ductility is somewhat lower than P91 ( 11\% (Webster et al., 2008)). With the exception of the interpretation of the PD data, all tests were performed in accordance with ASTM E1457-15.

\subsection{Experimental Testing: Methodology}

\subsubsection{Specimen Geometry}

All tests were performed on compact tension, $\mathrm{C}(\mathrm{T})$, specimens. This geometry is shown in
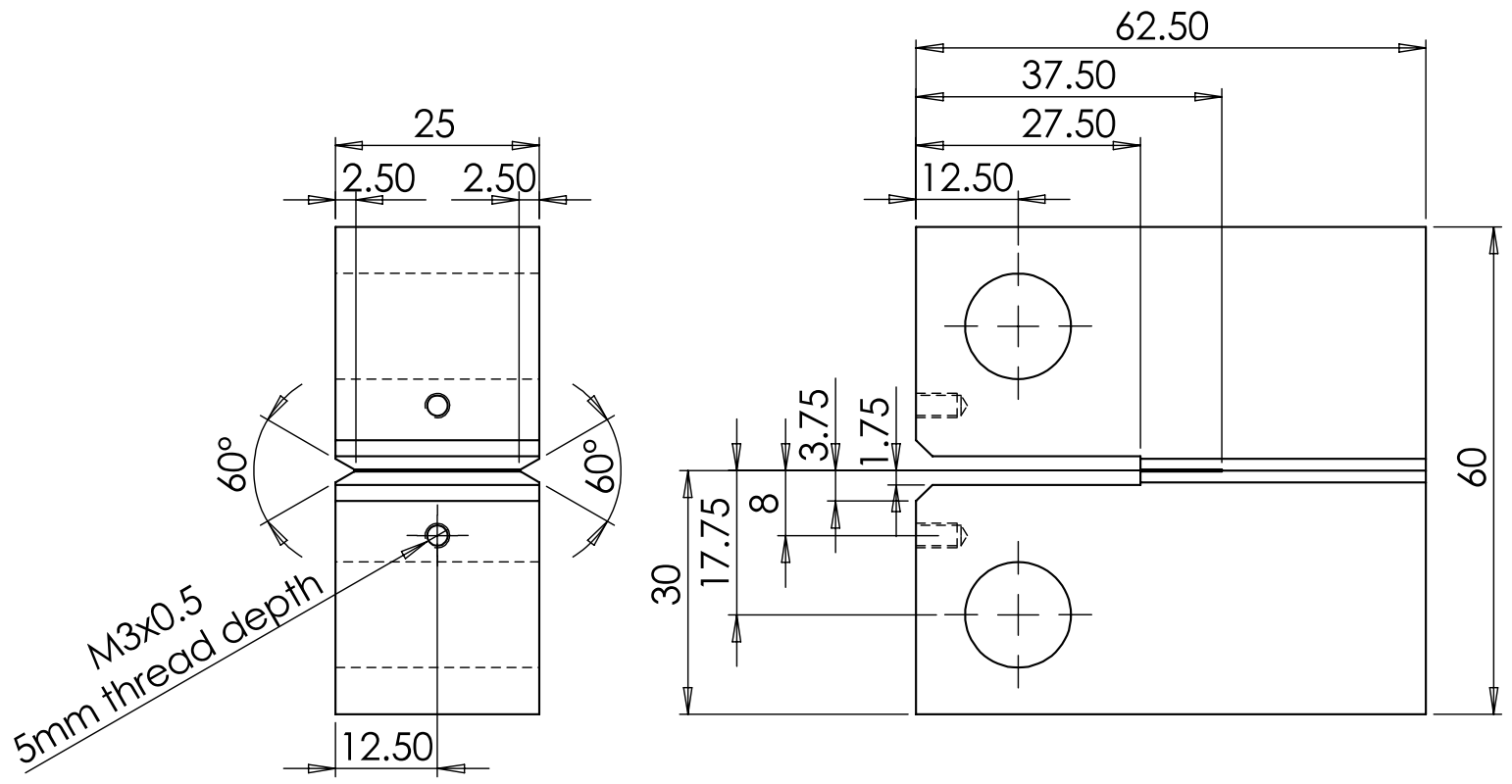

Figure 2 and the key dimensions are summarised in Table 1. The pre-crack was produced by $\mathrm{EDM}(0.15 \mathrm{~mm}$ tip radius).

\begin{tabular}{cccc}
\hline $\begin{array}{c}\boldsymbol{a}_{\mathbf{0}} \\
{[\mathbf{m m}]}\end{array}$ & $\begin{array}{c}\boldsymbol{W} \\
{[\mathbf{m m}]}\end{array}$ & $\begin{array}{c}\boldsymbol{B} \\
{[\mathbf{m m}]}\end{array}$ & $\begin{array}{c}\boldsymbol{B}_{N} \\
{[\mathbf{m m}]}\end{array}$ \\
\hline 25.0 & 50.0 & 25.0 & 20.0 \\
\hline
\end{tabular}

Table 1: C(T) specimen key dimensions.

\subsubsection{Extensometry}

For the Type $316 \mathrm{H}$ specimens the LLD was monitored using a capacitance gauge located in the crack mouth cut-out and the opposite face of the cut-out was used as the target for the gauge. For the P91 specimen the opposite face of the cut-out could not be used as the target 
due

to oxidation

so

the

fixture

shown

in

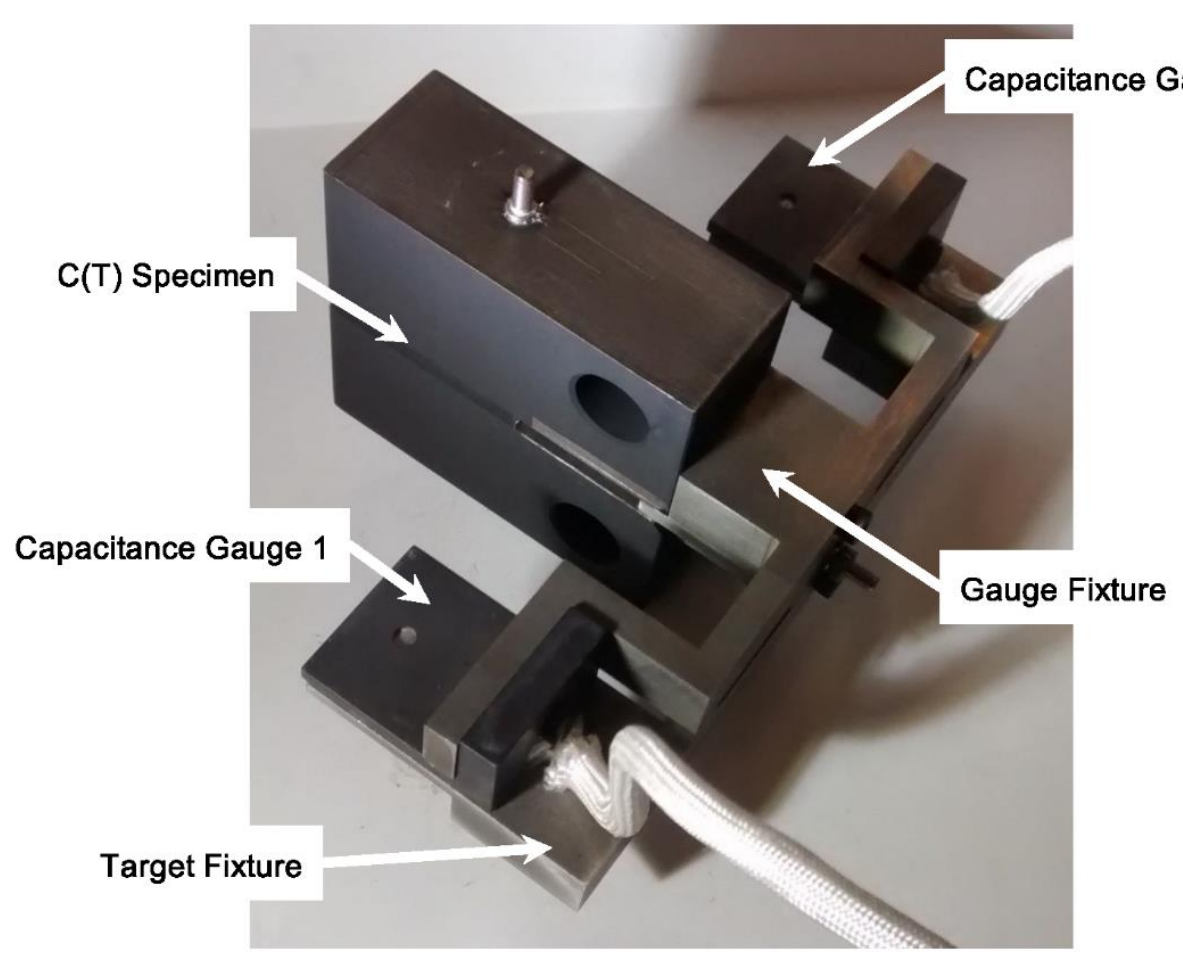

Figure 3 was manufactured from Nimonic 80A. This is a creep resistant nickel-based superalloy that does not experience significant oxidation at the test temperature of $620^{\circ} \mathrm{C}$. The gauge fixture was attached to one side of the crack mouth cut-out and the target fixture attached to the other side using the M3 holes shown in
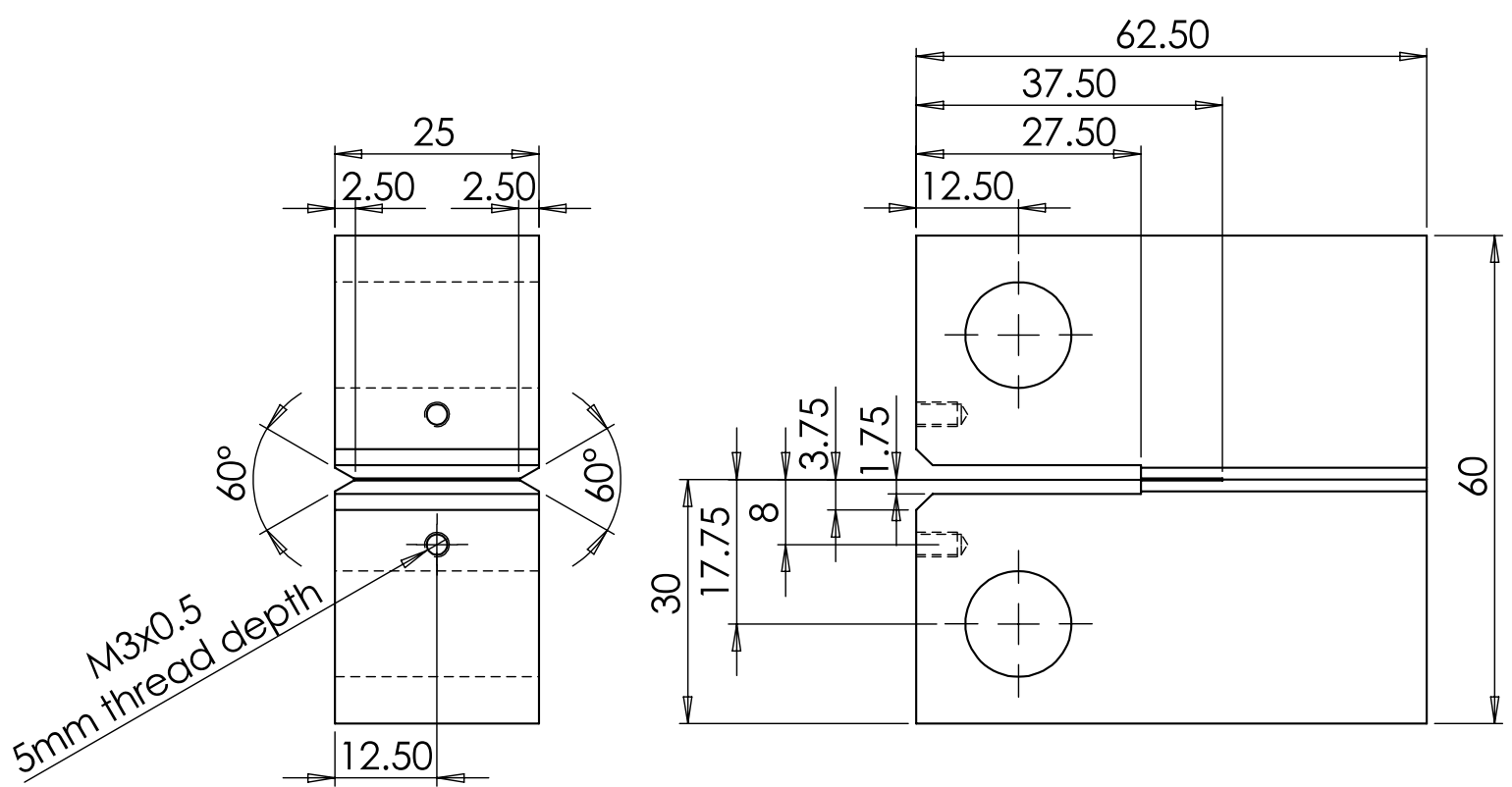

Figure 2. Two capacitance gauges were mounted on the fixture, as shown, and the LLD was calculated from the average of the two measurements. 


\subsubsection{Crack Extension Monitoring}

Crack extension was monitored using the PD technique. Direct Current Potential Drop (DCPD) is the most common method used to monitor CCG tests. At sufficiently low frequencies Alternating Current Potential Drop (ACPD) behaves in a quasi-DC manner. The frequency at which this occurs is material and geometry dependent. In this study a low frequency ACPD system, capable of frequencies as low as $2 \mathrm{~Hz}$, has been used where possible which demonstrates reduced noise and increased thermal stability compared to typical DCPD systems (Madhi and Nagy, 2011).

For the Type $316 \mathrm{H}$ specimens a quasi-DC current distribution was obtained at a frequency of $2 \mathrm{~Hz}$ so the ACPD system was used for these tests with a constant root mean square current of $3 \mathrm{~mA}$. For the P91 specimen a frequency of $2 \mathrm{~Hz}$ was not sufficiently low to obtain a quasi-DC current distribution due to the high magnetic permeability of this material so a DCPD system was used for this test with a constant current of 20A. For all tests the loading pins and specimen were coated with high temperature paint to electrically isolate the specimen from the testing machine, preventing alternative current paths.

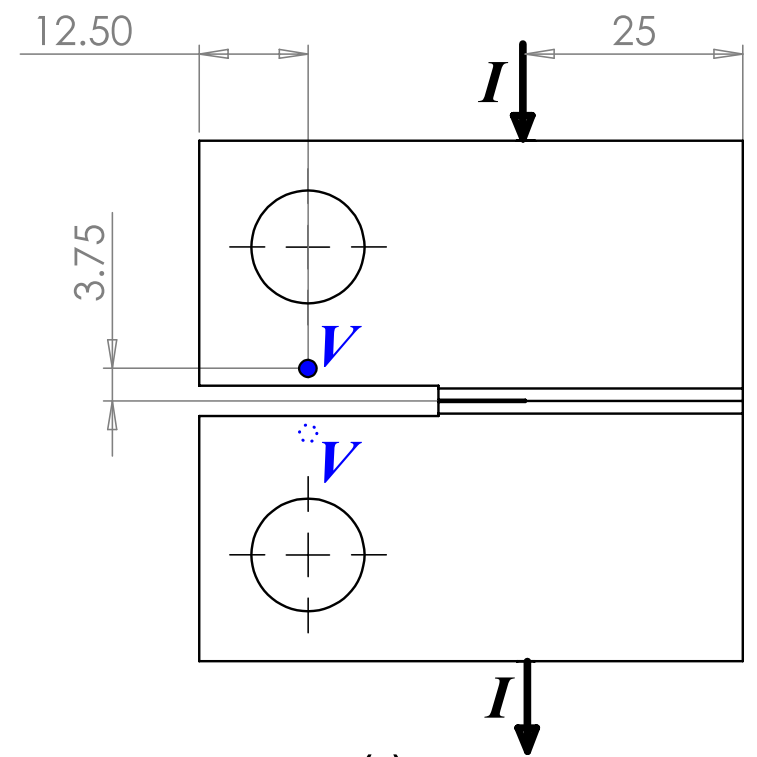

(a)

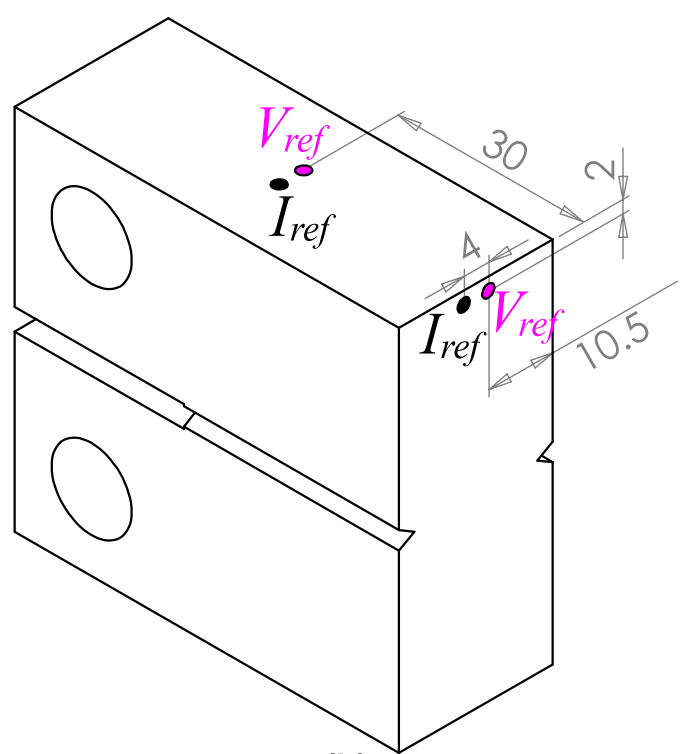

(b)

Figure 4(a) shows the configuration used to measure the PD across the crack. The PD probe locations, labelled ' $V$ ', are on opposite sides of the specimen, along the load-line, and either side of the crack. The current injection locations, labelled ' $I$ ', are at the mid-thickness of the top and bottom surfaces. A reference measurement, performed on the same specimen, was used to supress any fluctuations in temperature which occur throughout the test. The reference 


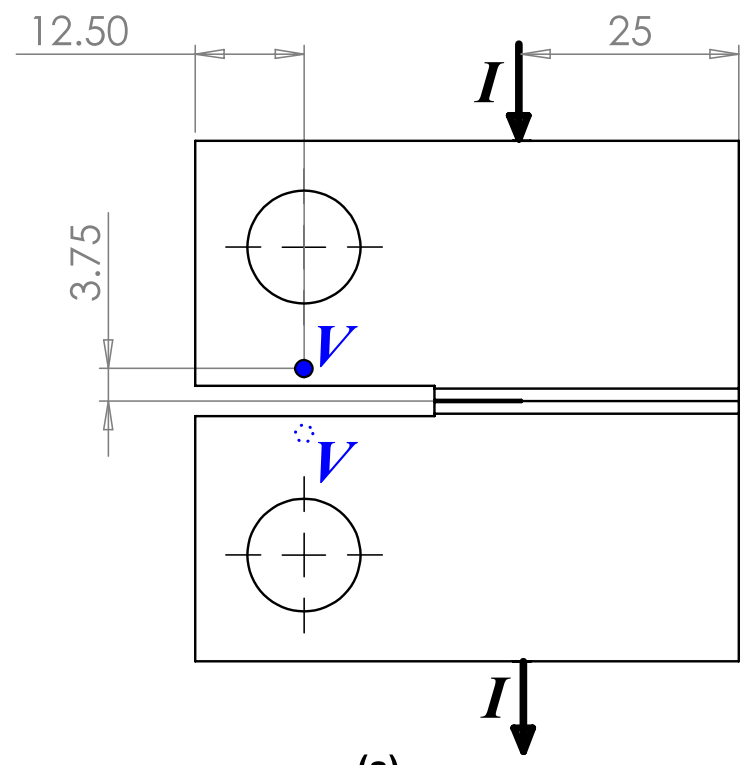

(a)

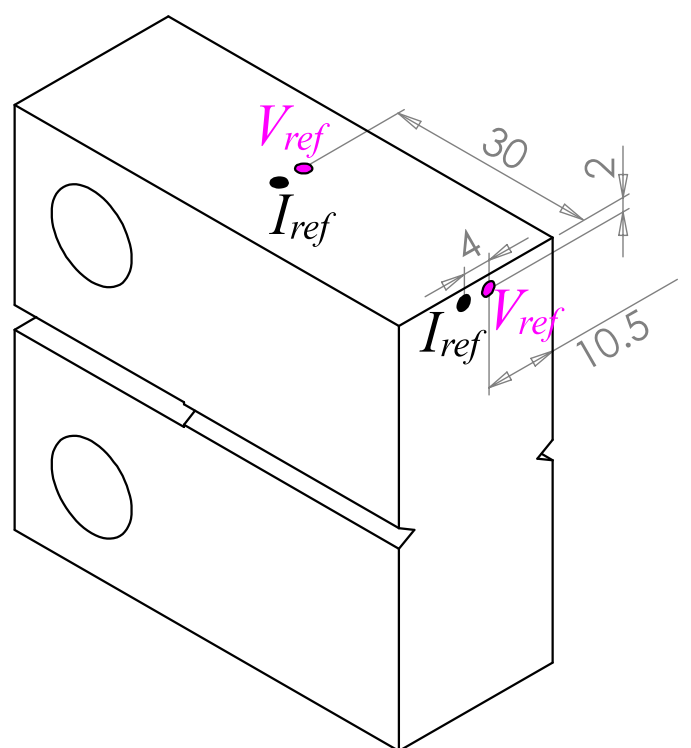

(b)

Figure 4(b) where the PD probe locations are labelled ' $V_{\text {ref }}$ ' and current injection locations are labelled ' $I_{r e f}$ '. This reference configuration has been shown to be relatively insensitive to crack extension (Tarnowski et al., 2014). The magnitude of the reference signal is approximately double the magnitude of the signal across the crack. This is because of the high current density caused by the proximity of the PD probes to the current injection leads.

A third order polynomial calibration function was derived for the PD configuration shown in

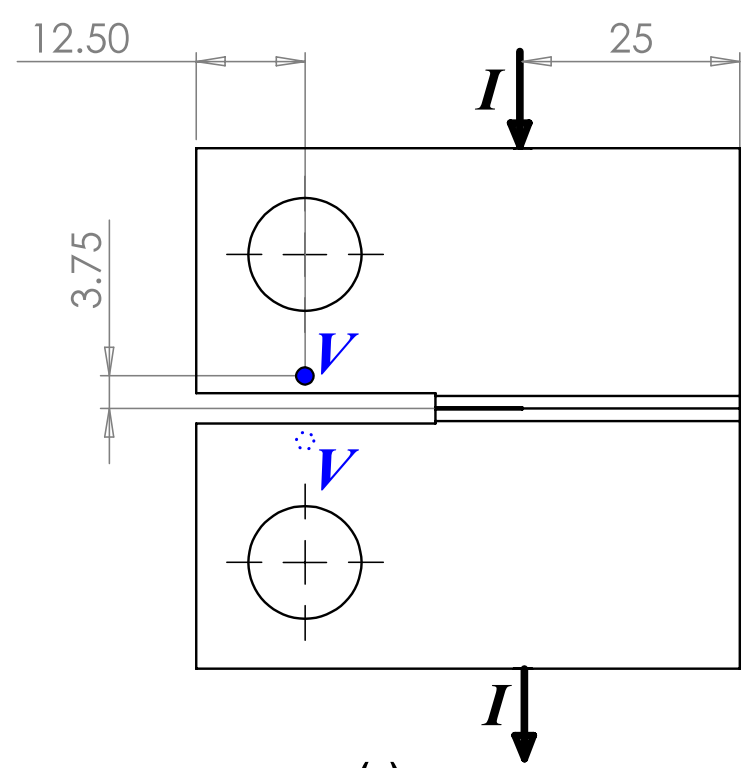

(a)

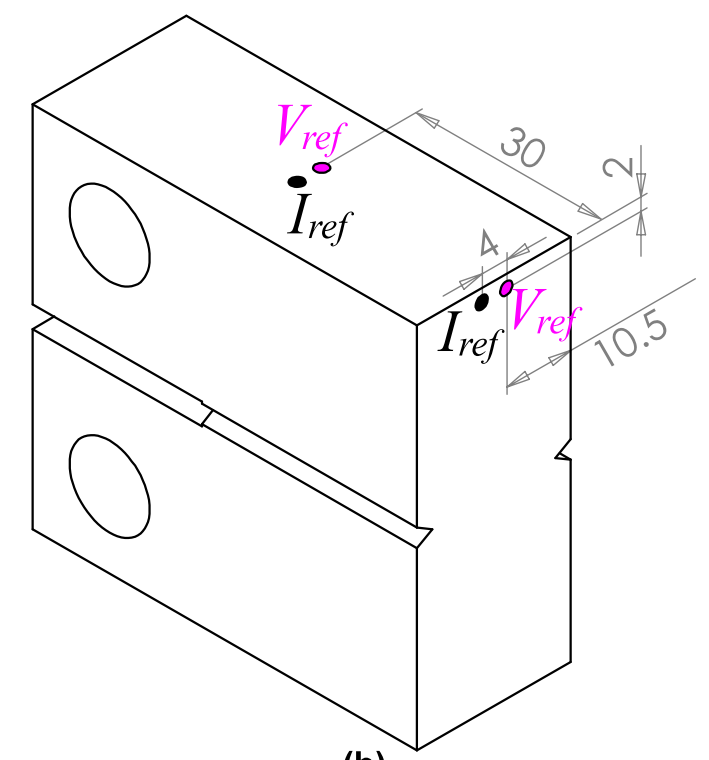

(b)

Figure 4(a) using COMSOL (COMSOL, 2012) and is provided in Equation (3). Unlike most DCPD systems, the low frequency ACPD system used for the majority of this study records 
resistance rather than PD however, for a constant current, the PD ratio in Equation (3), $V / V_{0}$, can be directly replaced by the resistance ratio $R / R_{0}$.

$$
\frac{a_{p}}{W}=-0.0839+0.8020\left(\frac{V}{V_{0}}\right)-0.2522\left(\frac{V}{V_{0}}\right)^{2}+0.0341\left(\frac{V}{V_{0}}\right)^{3}
$$

\subsubsection{Post-Test Sectioning}

After each test the specimen was sectioned by EDM as shown in

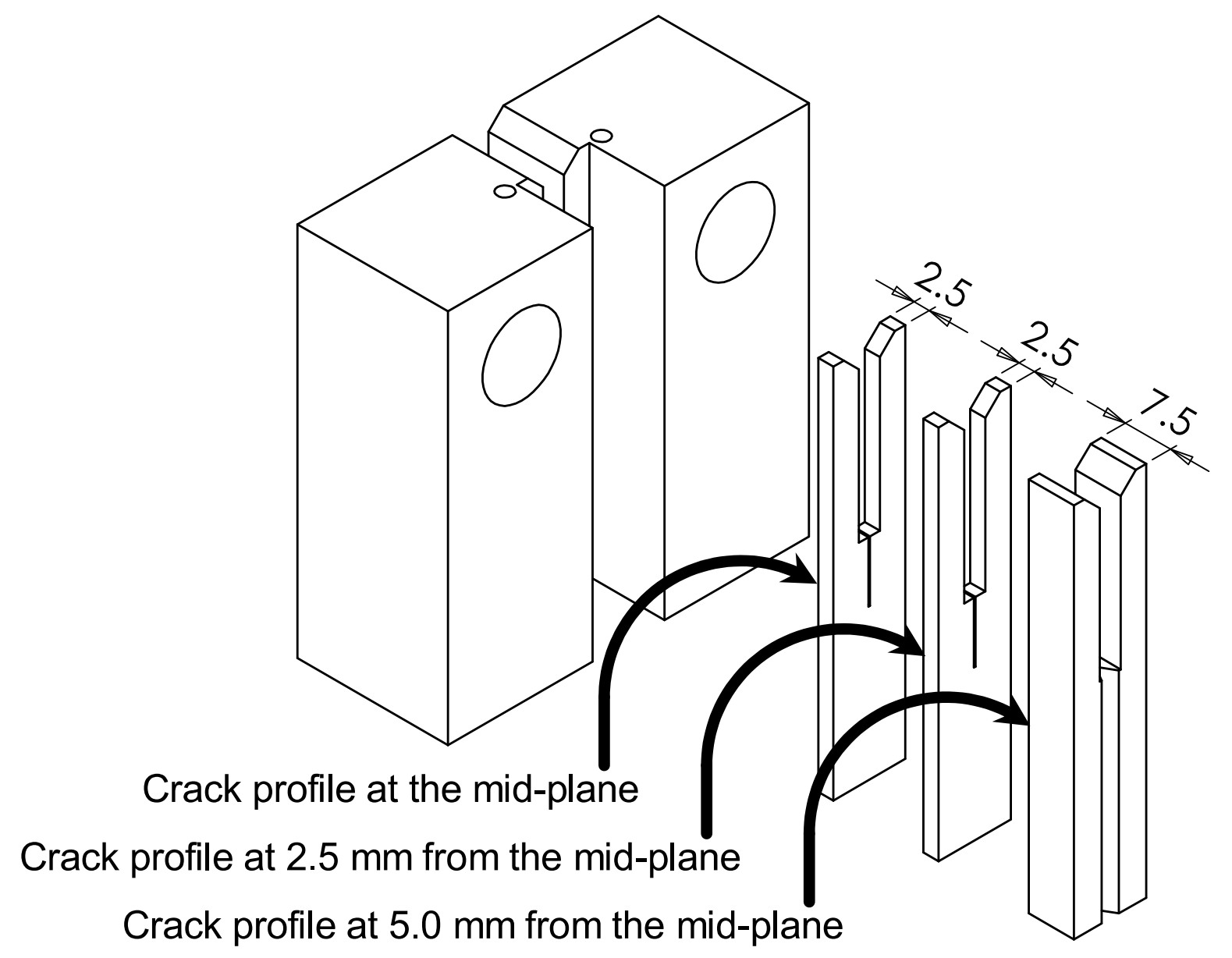

Figure 5. The thin sections were polished to reveal the crack profile at the mid-plane and at $2.5 \mathrm{~mm}$ and $5.0 \mathrm{~mm}$ from the mid-plane. This was observed using an optical microscope. The remaining specimen was fatigued open to reveal the fracture surface whilst minimising further deformation.

\subsection{Experimental Testing: Preliminary Test}

An interrupted CCG test was performed on ferritic P91 steel at $620^{\circ} \mathrm{C}$ to investigate the significance of creep strains on PD measurements in the absence of crack extension. The load applied to the specimen was $12.0 \mathrm{kN}$. This results in a reference stress of $93.2 \mathrm{MPa}$, assuming 
plane strain conditions. This is $39 \%$ of the $0.2 \%$ proof stress at $620^{\circ} \mathrm{C}(240 \mathrm{MPa}$ (NIMS, 2014)) so the plastic zone should be limited to a small region at the crack tip.

Microstructural evolution of P91 at high temperature is a well-documented phenomenon, e.g. (Orlova et al., 1998; Panait et al., 2010), and can influence PD measurements. To mitigate this, the specimen was thermally soaked for 570 hours prior to loading to allow the PD signal to stabilise. The load was applied for 1660 hours before the test was interrupted and the PD measurements interpreted according to ASTM E1457-15. The crack extension predicted from the PD signal, $\Delta a_{p}$, using the calibration function in Equation (3), is plotted against time in
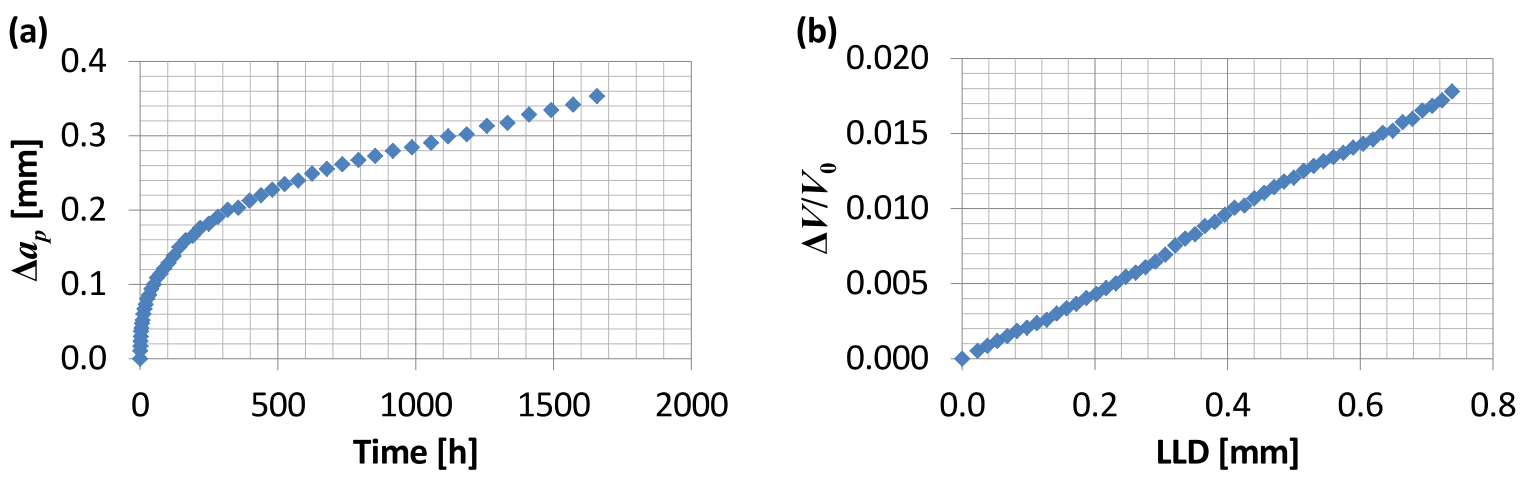

Figure 6(a) and relative change in PD vs. LLD is shown in
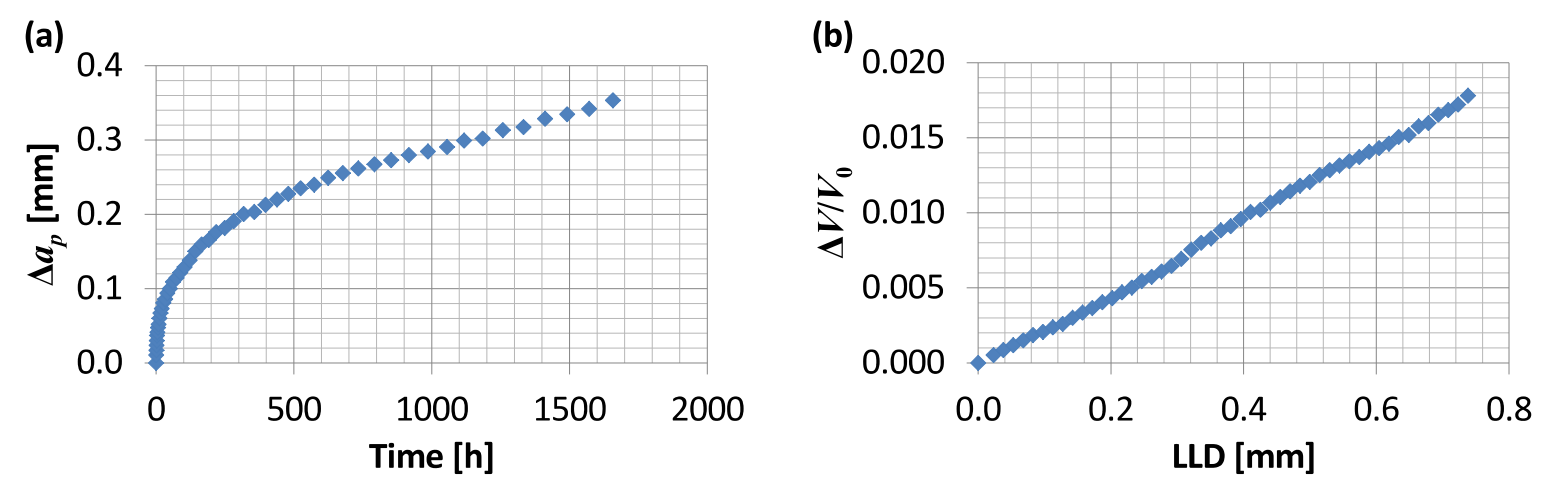

Figure 6(b). The crack tip profile at the various points through the thickness is shown in 


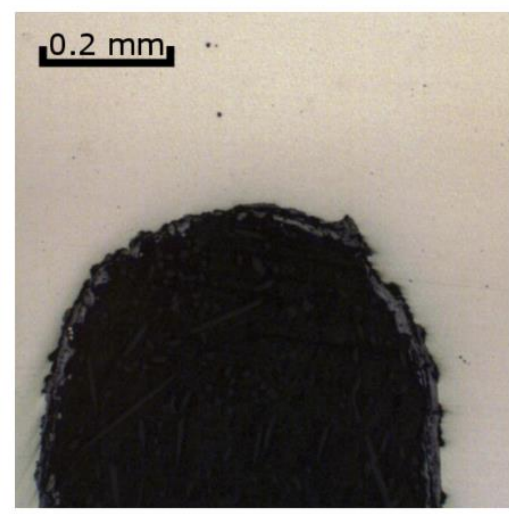

(a)

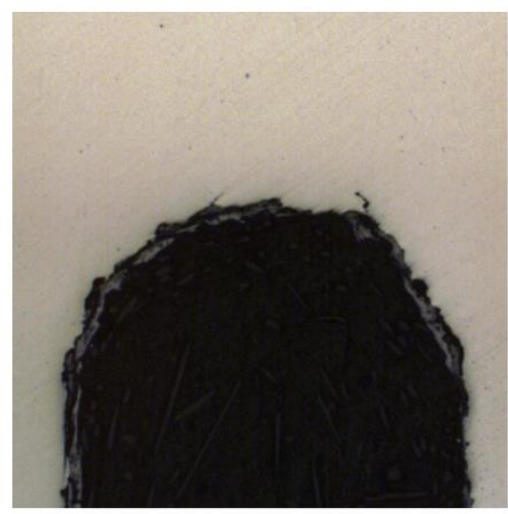

(b)

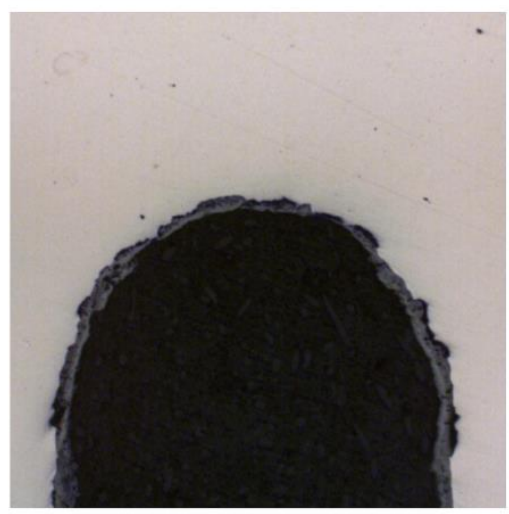

(c)

Figure 7.

When the PD data is interpreted in accordance with ASTM E1457-15, the $0.2 \mathrm{~mm}$ 'engineering definition' of crack initiation is predicted to occur after $\sim 300$ hours and at the termination of the test $\sim 0.35 \mathrm{~mm}$ of crack extension is predicted (see
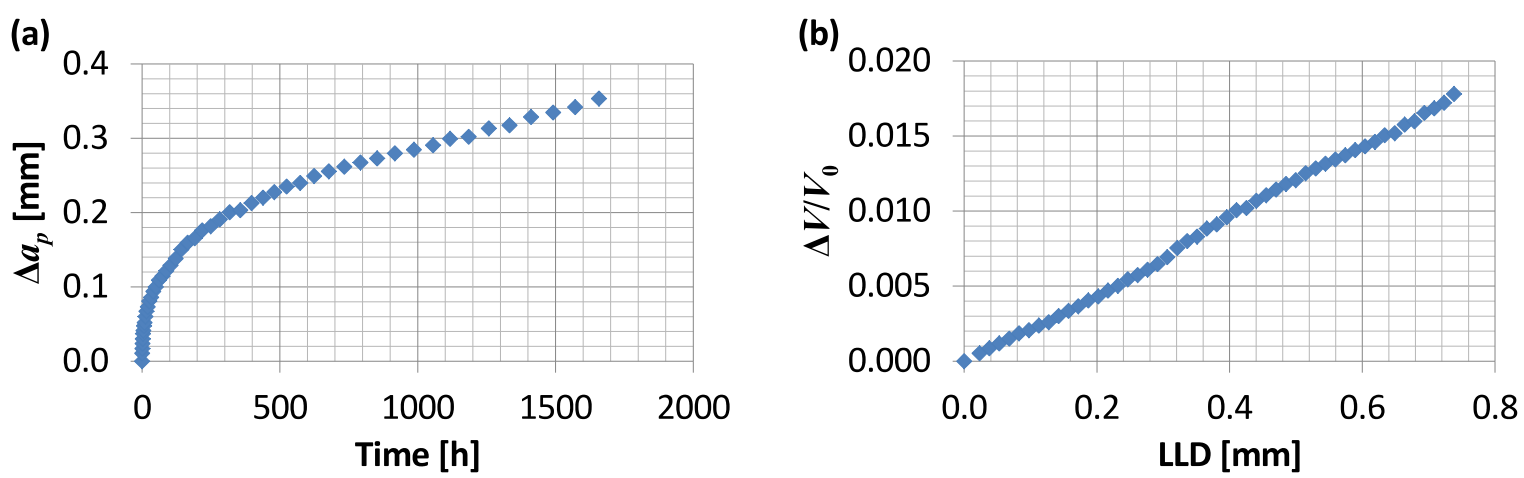

Figure 6(a)). Despite these predictions, there is no evidence of any crack growth from the EDM pre-crack (see

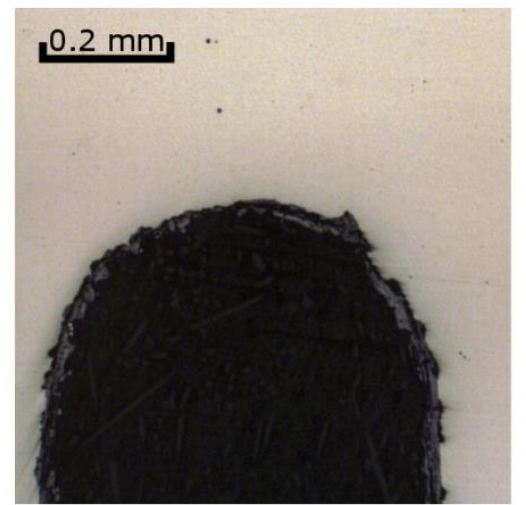

(a)

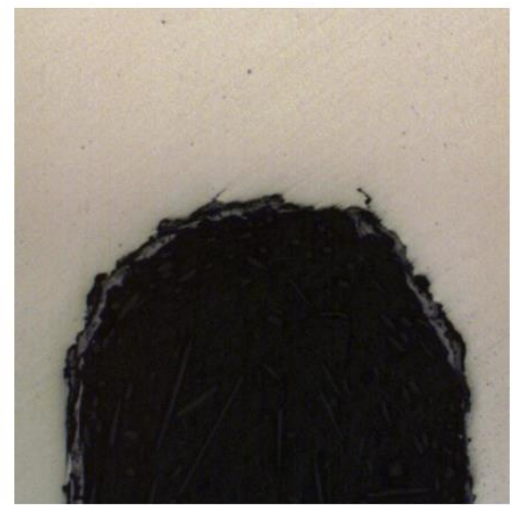

(b)

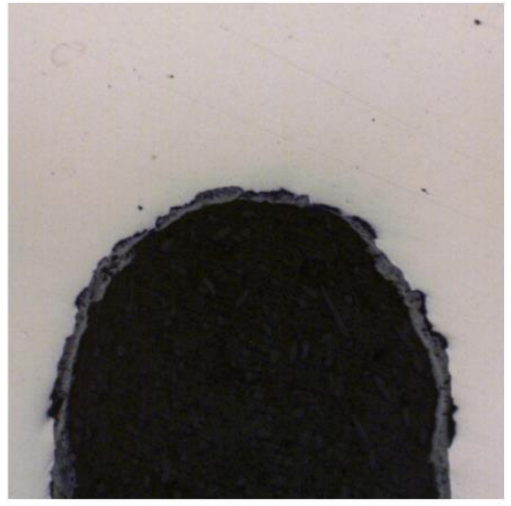

(c) 
Figure 7). The incubation period of this specimen must therefore longer than the 1660 hour duration of the test, so the prediction based on the PD of $\sim 300$ hours is underestimated by at least a factor of 5.5 .

It is apparent from

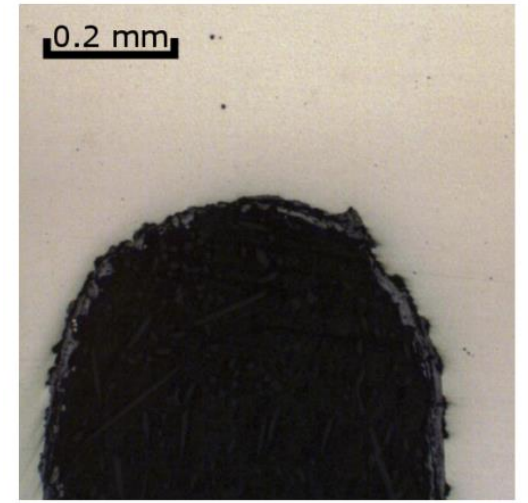

(a)

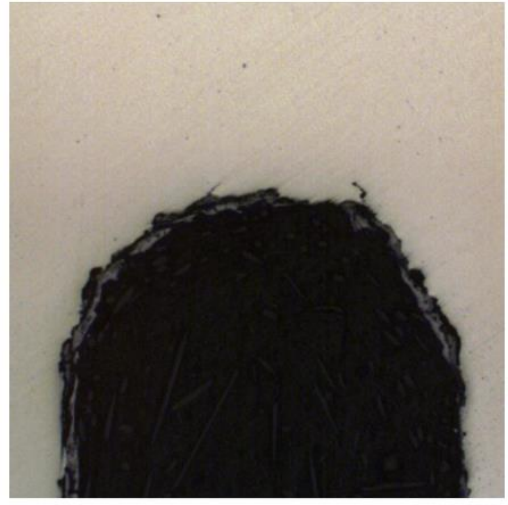

(b)

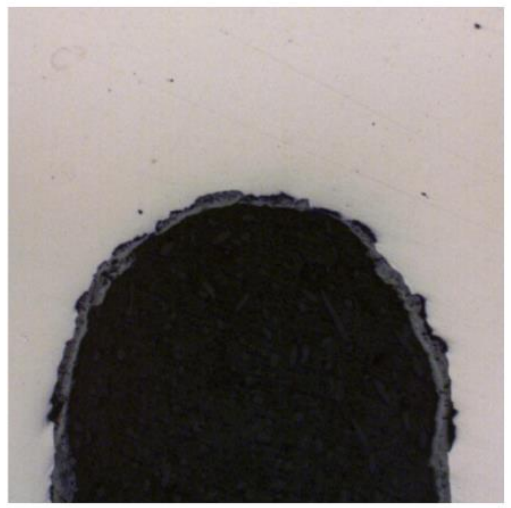

(c)

Figure 7 that significant permanent deformation has occurred throughout the test. The diameter of the notch tip is $\sim 0.6 \mathrm{~mm}$ which is double the width of the original EDM pre-crack. Given that the reference stress is small compared to the $0.2 \%$ proof stress, the majority of this deformation is most likely due to creep rather than plasticity on loading. This creep strain is the likely cause of the spurious crack extension measurement.

This preliminary test has shown that the PD technique, as defined in ASTM E1457-15, can significantly overestimate crack growth and underestimate the incubation period for tests performed on creep ductile materials. This highlights the need for an alternative method of interpreting PD data such as the one proposed here. Furthermore, the relationship between PD and LLD, shown in
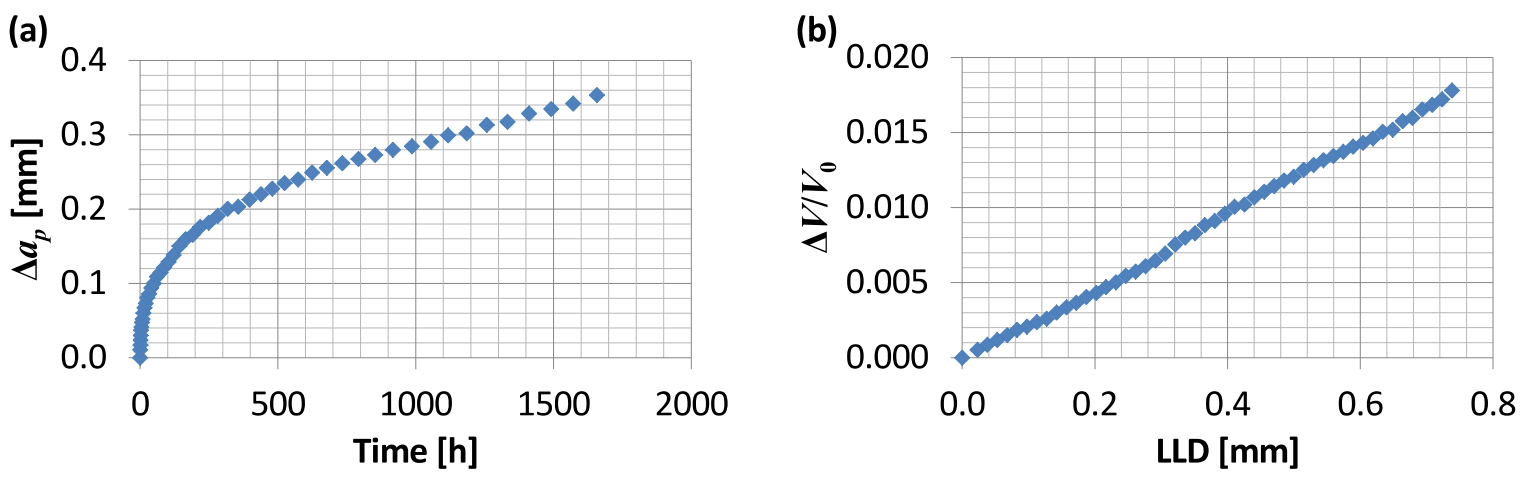

Figure 6(b), shows no increase in gradient which is consistent with the lack of crack growth observed from the crack profiles and the hypothesis that such an increase will only occur at the 
onset of crack growth. This is investigated further using interrupted tests in the following section.

\subsection{Experimental Testing: Interrupted Tests}

A series of CCG tests were performed on specimens manufactured from ex-service Type $316 \mathrm{H}$ (cast 55882) at $550{ }^{\circ} \mathrm{C}$ with a load, $P$, of $24.5 \mathrm{kN}$. This corresponds to a reference stress of $190 \mathrm{MPa}$, assuming plane strain conditions, which is approximately the $0.2 \%$ proof stress (191.9 MPa (BEGL, 2000)). These tests have been performed using austenitic stainless steel to capitalise on the reduced noise and increased thermal stability of the low frequency ACPD system. The low magnetic permeability of this material ensures that a quasi-DC current distribution can be obtained. This material also avoids the additional complication of microstructural evolution which can influence PD measurements performed on P91 at high temperature. It is therefore ideal for unambiguously verifying the proposed method of interpreting PD data from CCG tests.

An initial test was performed to see if an increase in gradient on a plot of PD against LLD is observed which may indicate the onset of crack growth. During this test, crack growth was monitored using DCPD and low frequency ACPD simultaneously to confirm that the two systems demonstrate the same response. The test was continued until final failure. The results are shown in

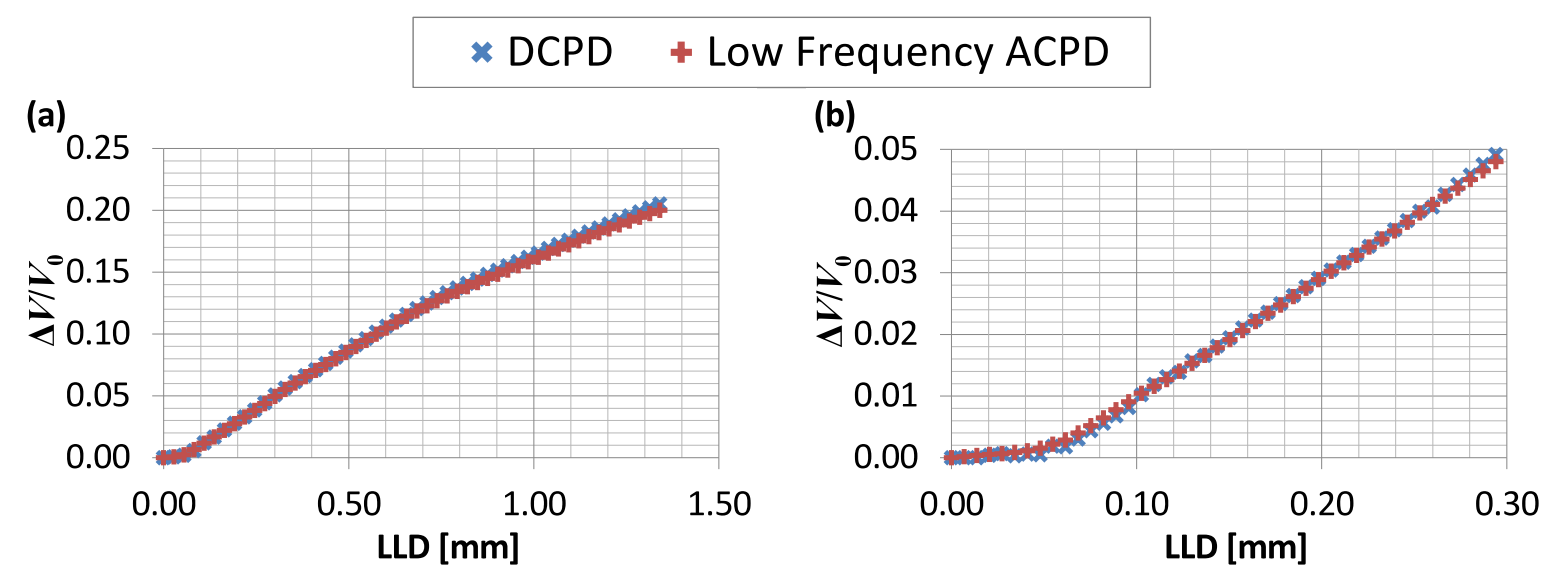

Figure 8. 

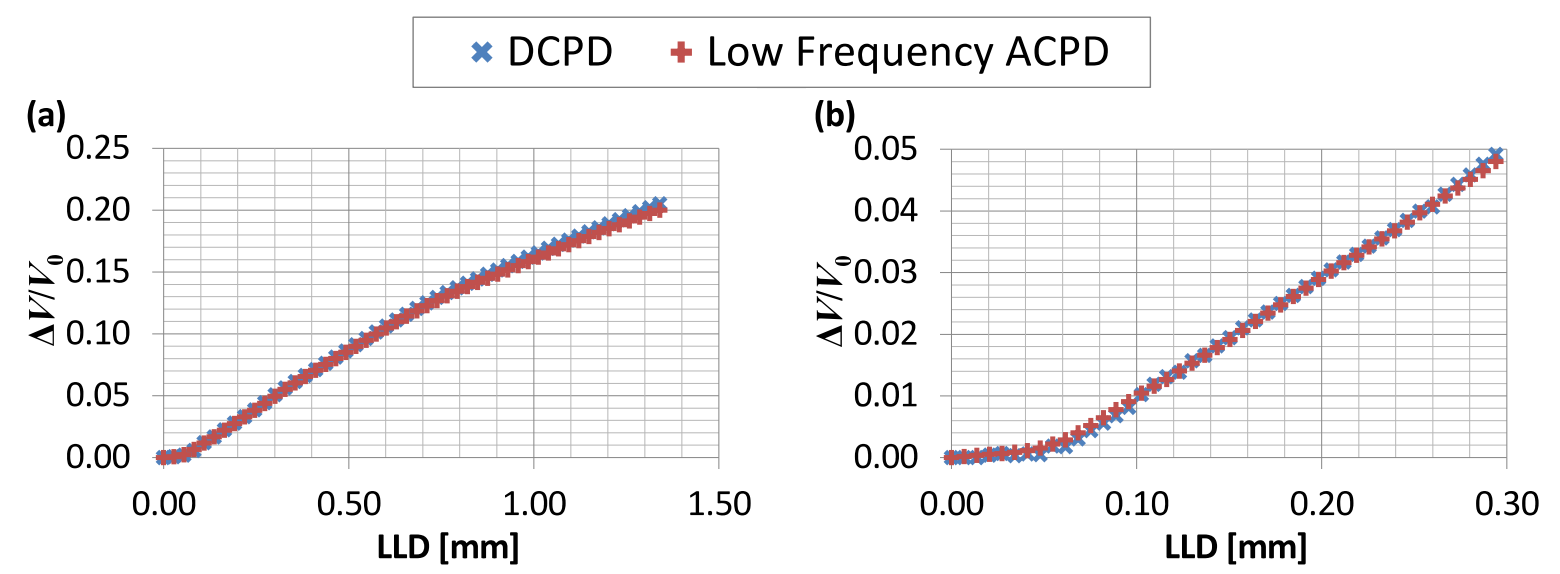

Figure 8(a) shows the relationship between relative change in PD and LLD for the entire test, and

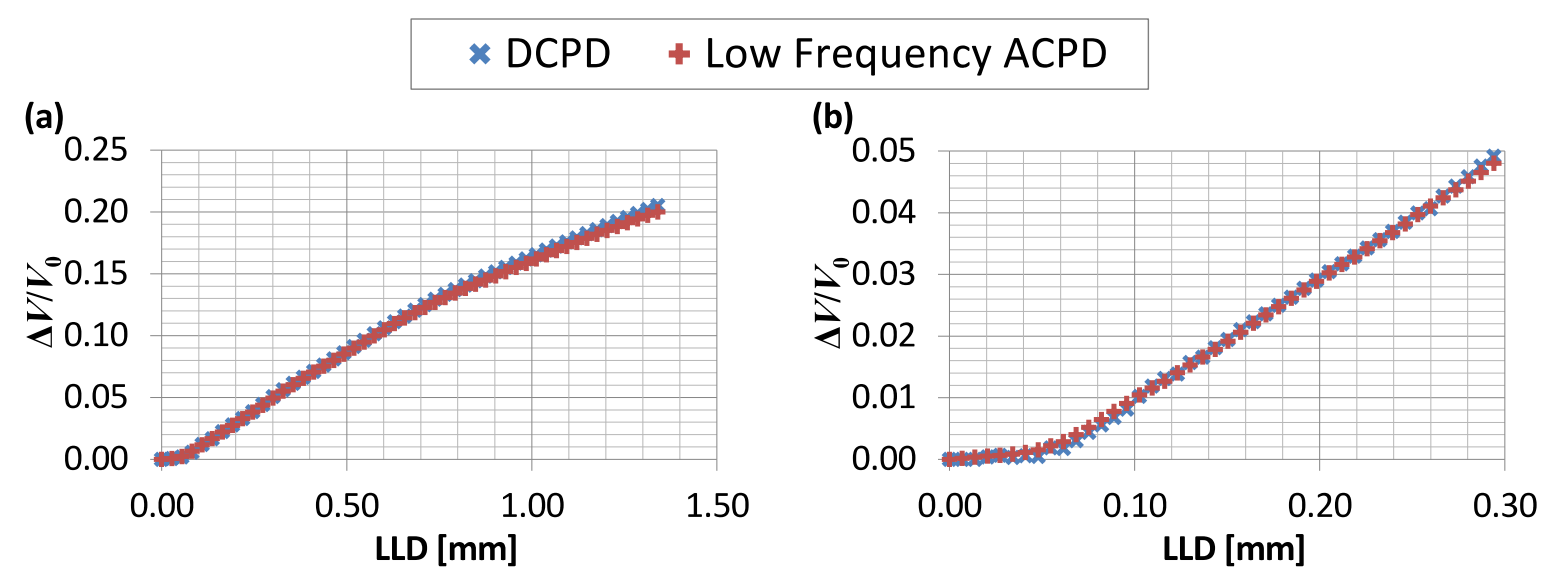

Figure $8(\mathrm{~b})$ focuses the initial part of the test.

There is good agreement between the low frequency ACPD system and the DCPD system for the entire duration of the test which confirms that the ACPD system is behaving in a quasi-DC manner. Both systems demonstrate an increase in gradient at a LLD of $\sim 0.06 \mathrm{~mm}$ which may correspond to crack initiation. This occurred after $\sim 230$ hours. To investigate if this change in gradient corresponds to crack initiation, a series of nominally identical tests have been performed which were interrupted at different stages as detailed in Table 2.

\begin{tabular}{cl}
\hline Specimen ID & \multicolumn{1}{c}{ Point of Interruption } \\
\hline CCG316_CT01 & After significant crack growth but prior to final failure. \\
CCG316_CT02 & After $~ 0.2$ mm of crack extension is predicted by the PD. \\
CCG316_CT03 & Immediately after the increase in gradient on a plot of PD vs. LLD. \\
CCG316_CT04 & Immediately after the increase in gradient on a plot of PD vs. LLD. \\
CCG316_CT05 & Prior to the increase in gradient on a plot of PD vs. LLD. \\
\hline
\end{tabular}


CCG316_CT06 Prior to the increase in gradient on a plot of PD vs. LLD.

Table 2: Point of interruption for each interrupted CCG test.

\subsubsection{Interrupted Tests Results}

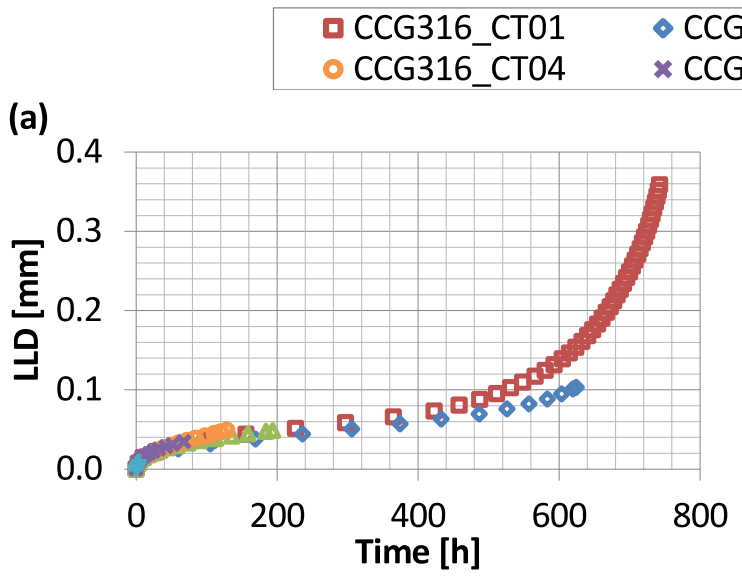

$\begin{array}{ll}\text { CCG316_CT02 } & \triangle \text { CCG316_CT03 } \\ \times \text { CCG316_CT05 } & + \text { CCG316_CT06 }\end{array}$

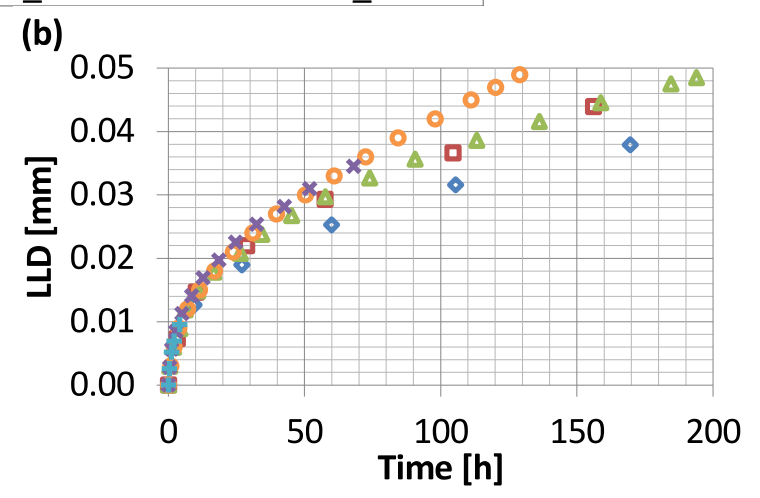

Figure 9(a) shows the LLD for the duration of each test and

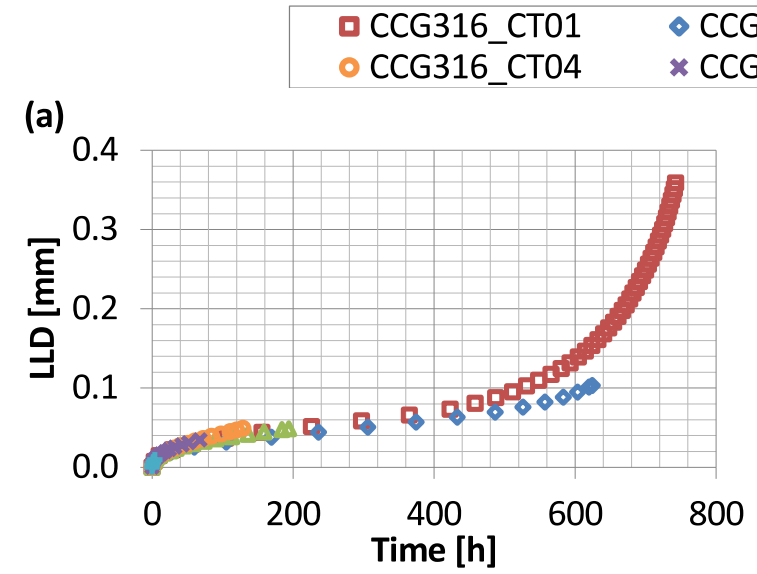

\begin{tabular}{ll}
\hline CTO2 & $\triangle$ CCG316_CT03 \\
\hline _CT05 & + CCG316_CT06
\end{tabular}

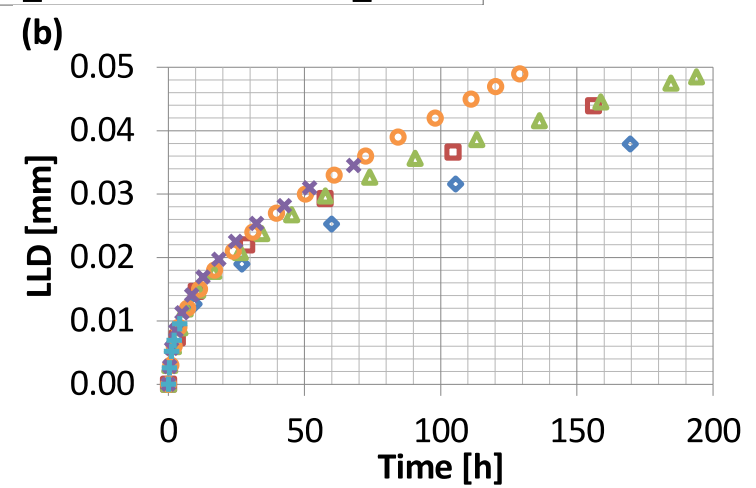

Figure 9(b) shows the same data for the initial 200 hours. Although there is some scatter, which is typical of CCG testing, the response of all six specimens is similar which suggests relatively consistent creep properties throughout the ex-service material. 


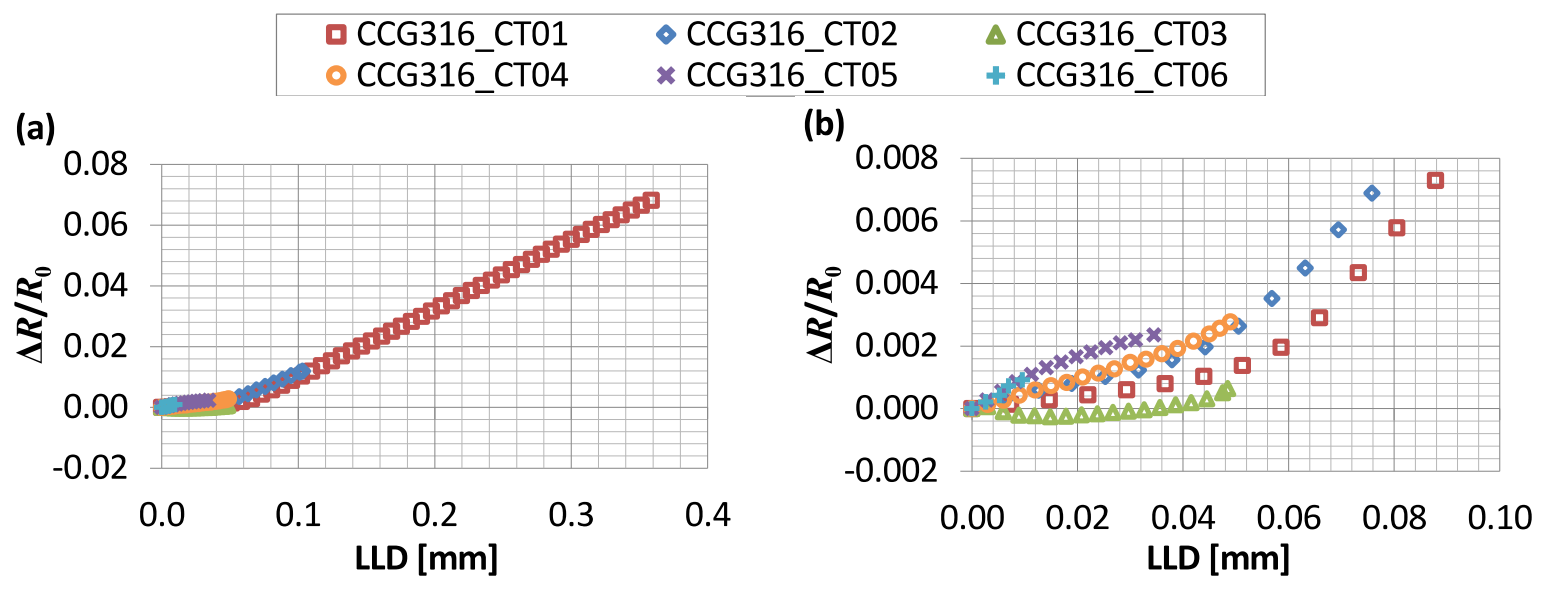

Figure 10(a) shows the relative change in resistance with LLD for the duration of each test whilst
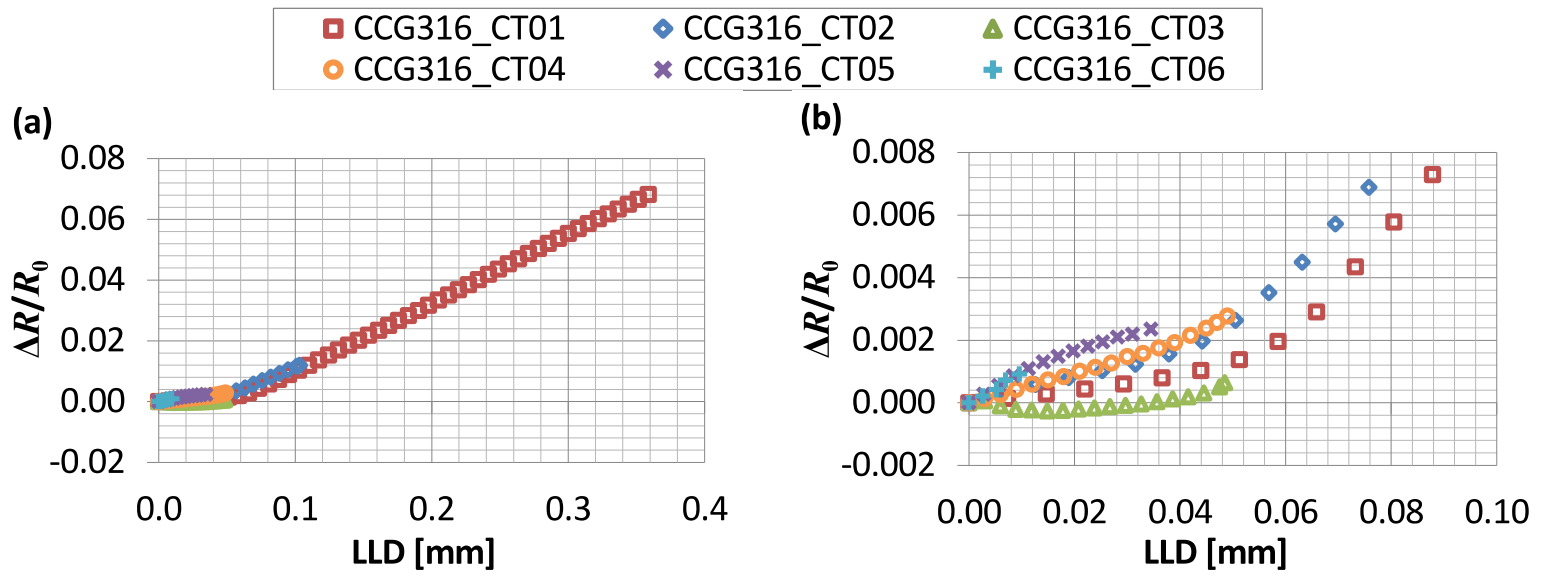

Figure 10(b) shows the same data for the initial part of the test. The change in resistance is relative to the value at the start of the test immediately after load-up, $R_{0}$. Plotting the resistance in this format allows all six data sets to be compared on the same plot. Each specimen demonstrates a similar general trend although there are small differences in the initial response. Of the four tests for which an increase in gradient is observed (specimens CCG316 _CT01 to CCG316_CT04) this increase consistently occurs at a LLD between $0.04 \mathrm{~mm}$ and $0.06 \mathrm{~mm}$. 

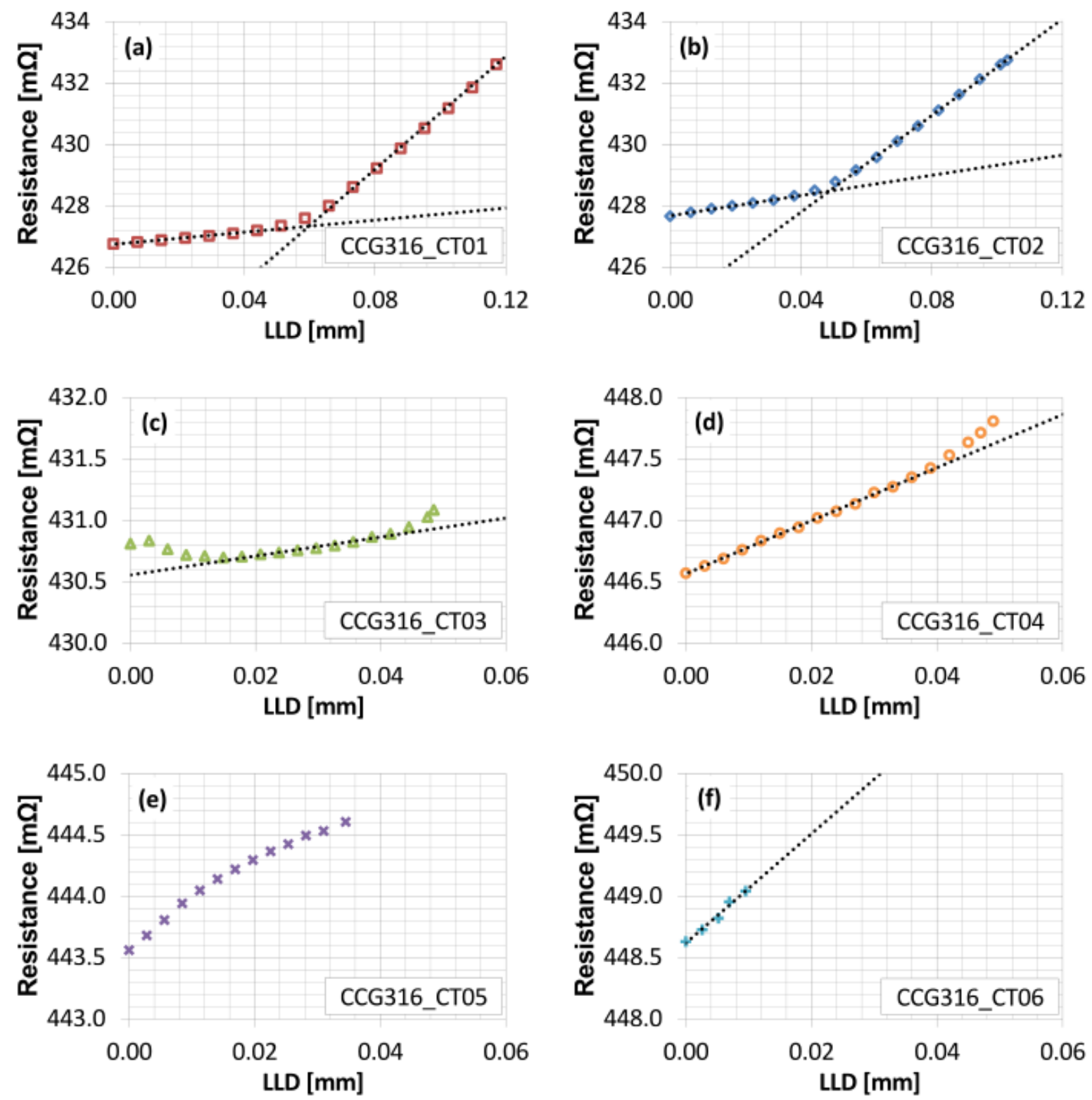

Figure 11 provides separate plots for each test showing the relationship between resistance and LLD. Only the initial part of each test is shown including, where applicable, the increase in gradient. In 

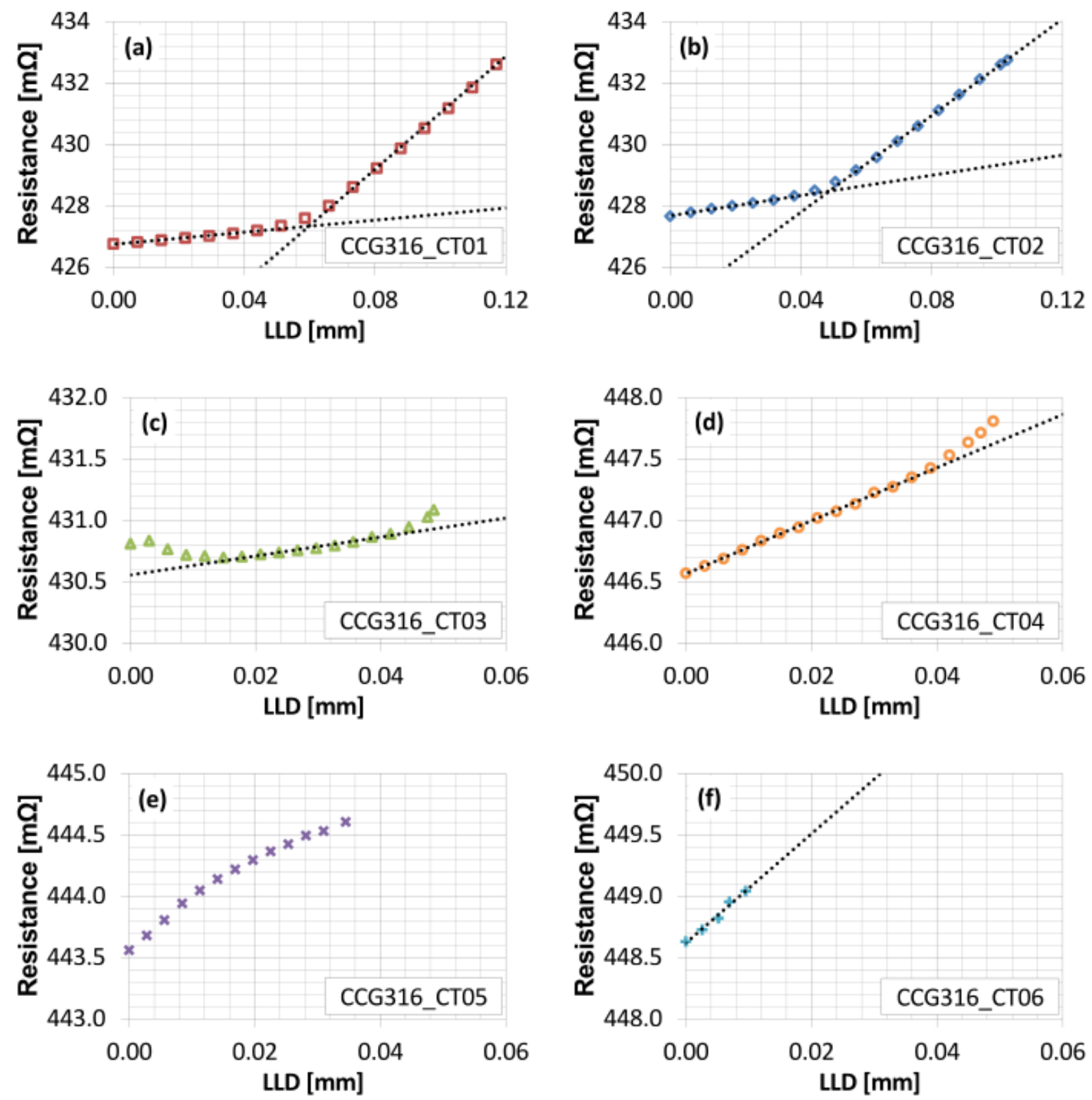

Figure 11(a) and (b) (specimens CCG316_CT01 and CCG316_CT02) the data either side of the increase in gradient is approximately linear as shown by regression fits. In 

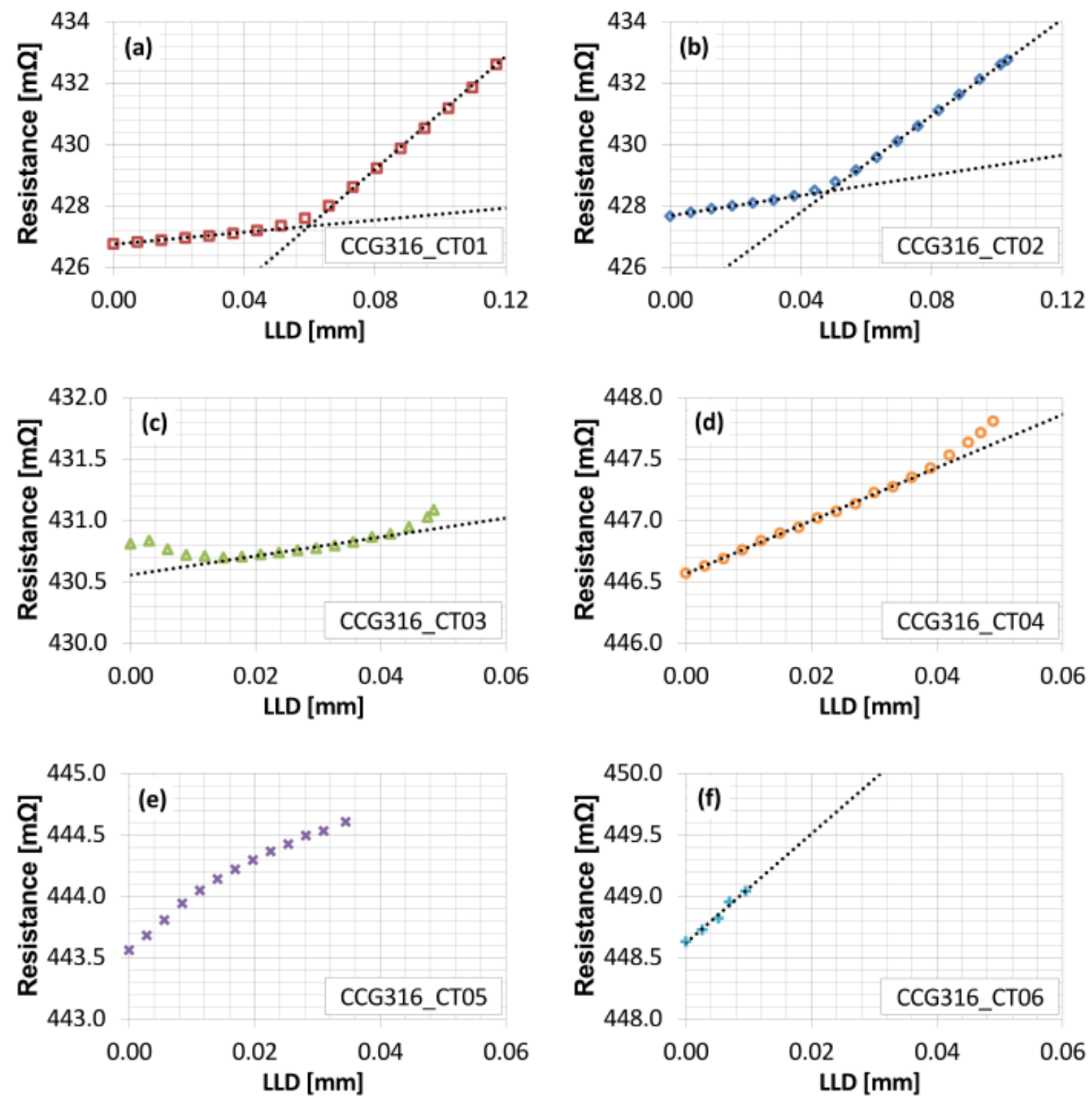

Figure 11(c) and (d) (specimens CCG316_CT03 and CCG316_CT04) the data before the increase in gradient is approximately linear which is again shown by regression fits. This is with the exception of an initial drop in PD observed for specimen CCG316_CT03, which is not uncommon during CCG tests (Freeman and Neate, 1980) however, this drop occurs over the initial 8 hours of the test so it does not mask the increase in gradient which occurs after 94 hours. For specimens CCG316_CT03 and CCG316_CT04 there is insufficient data after the increase in gradient to identify any trend. 

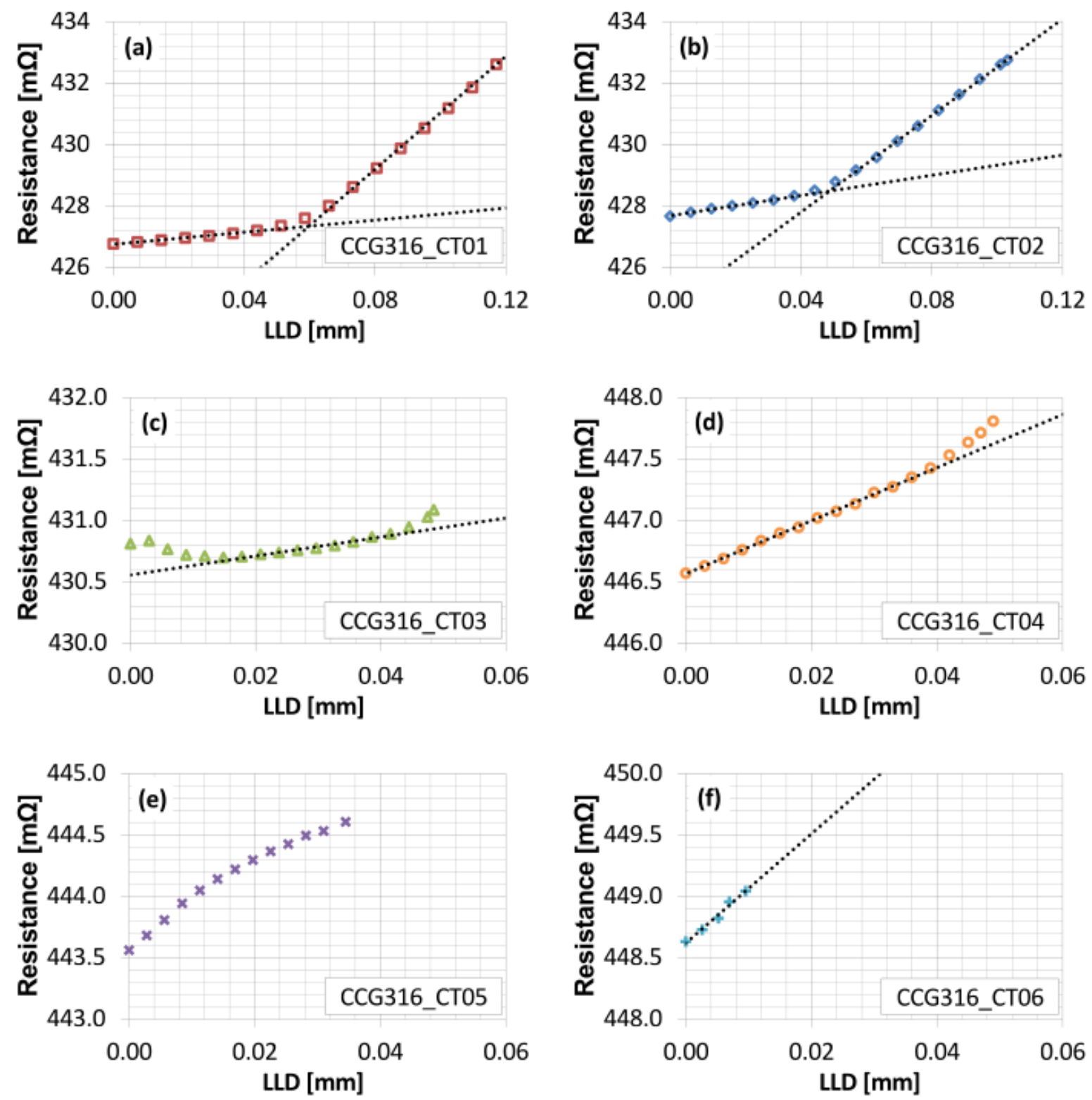

Figure 11(f) (specimen CCG316_CT06) also demonstrates a linear trend, shown by a regression fit, but in 

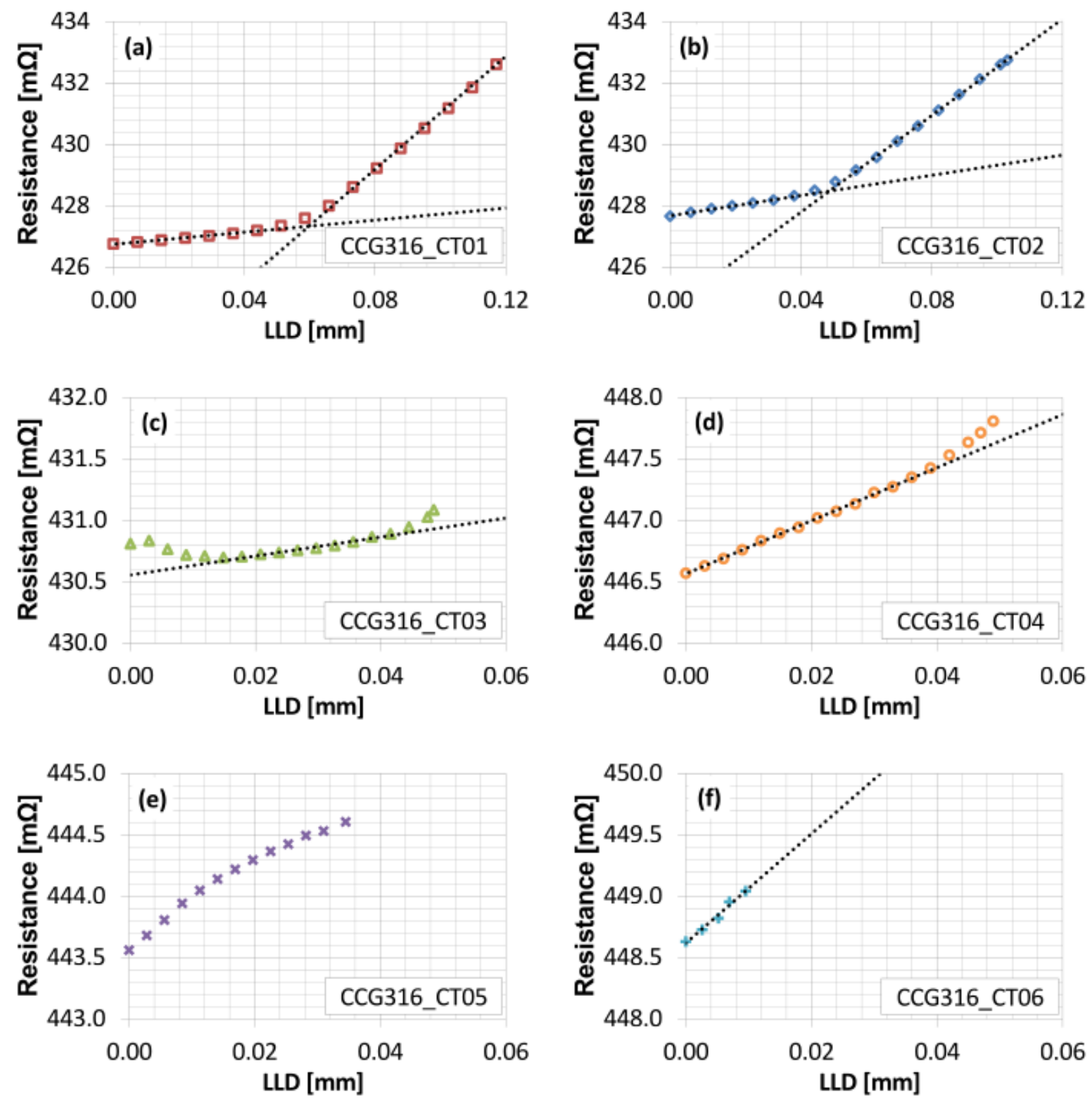

Figure 11(e) (specimen CCG316_CT05) the trend is slightly non-linear with a small reduction in gradient so no regression fit has been applied to this data. Despite this slightly different behaviour, any increase in gradient, of the order observed for specimens CCG316_CT01 to CCG316_CT04, would still be apparent.

Optical microscope images of the crack profiles at the mid-plane and $2.5 \mathrm{~mm}$ and $5.0 \mathrm{~mm}$ from the mid-plane are provided for all six specimens in 


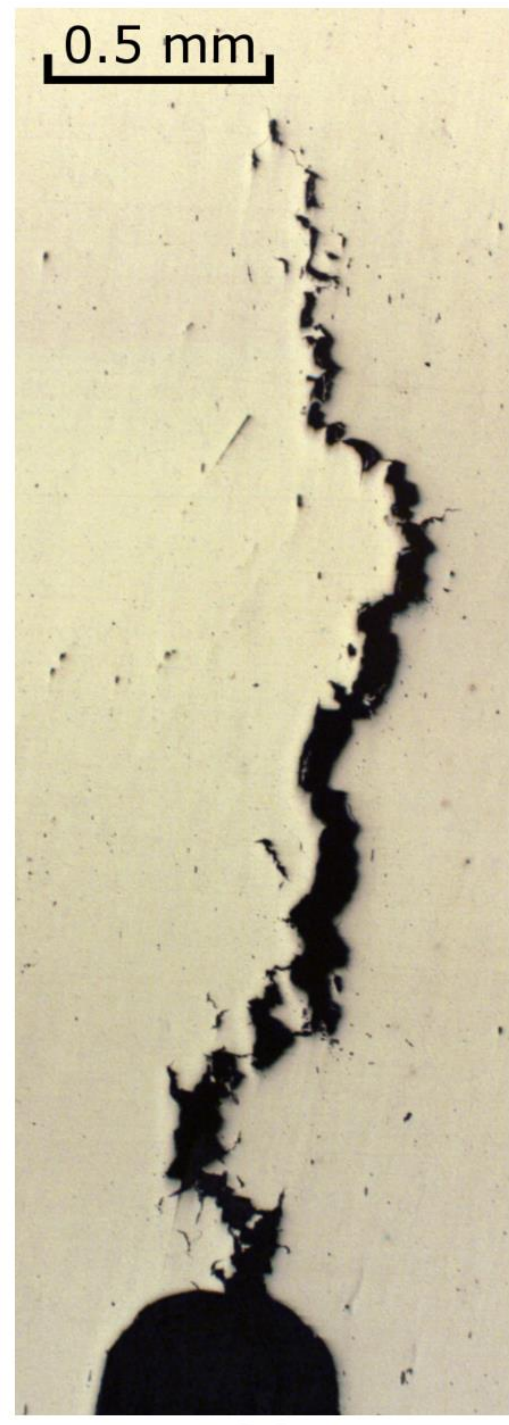

(a)

Figure

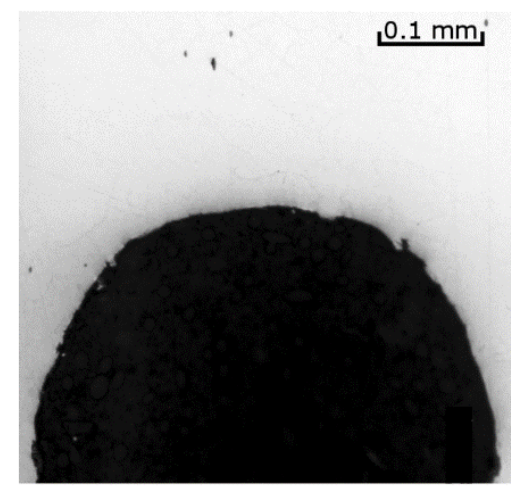

(a)

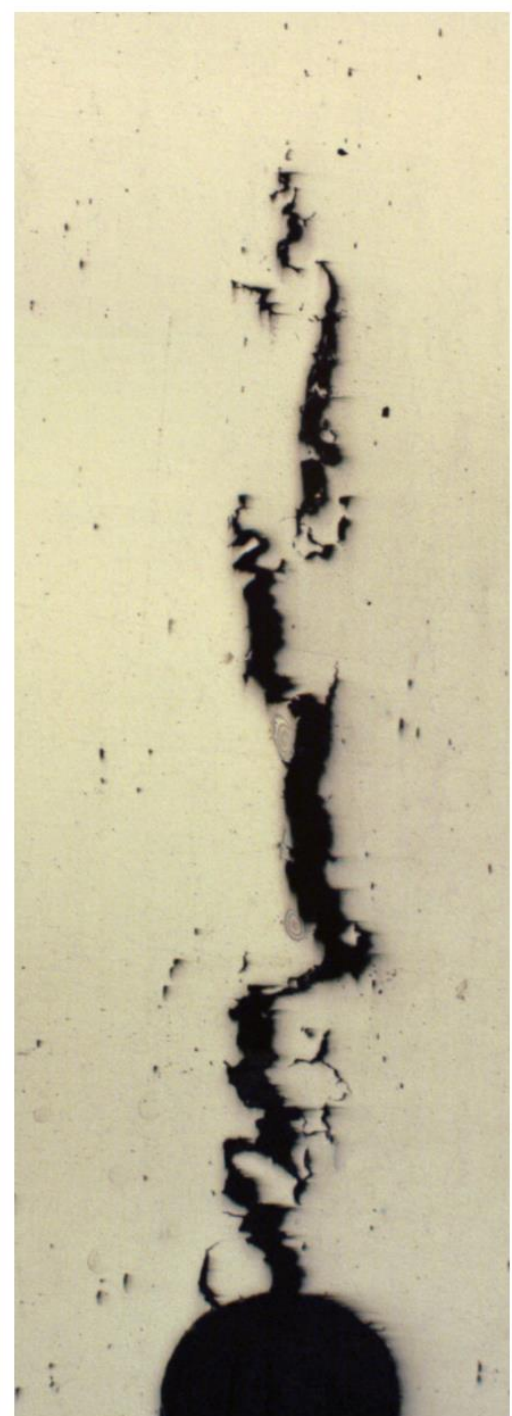

(b)

12

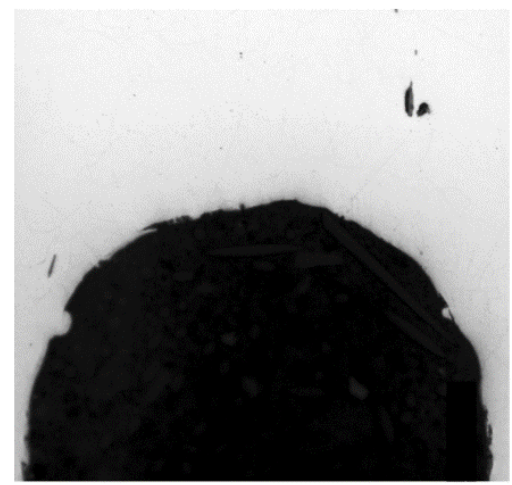

(b)

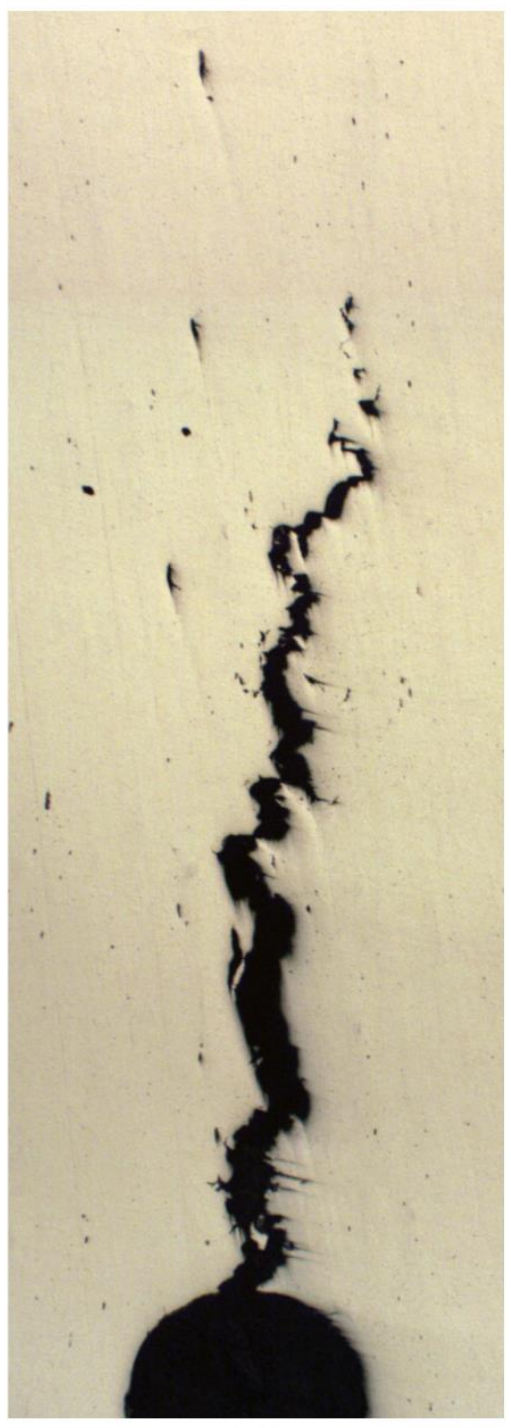

(c) to

Figure 17. Significant crack growth occurred in specimens CCG316_CT01 and CCG316_CT02 as observed in 


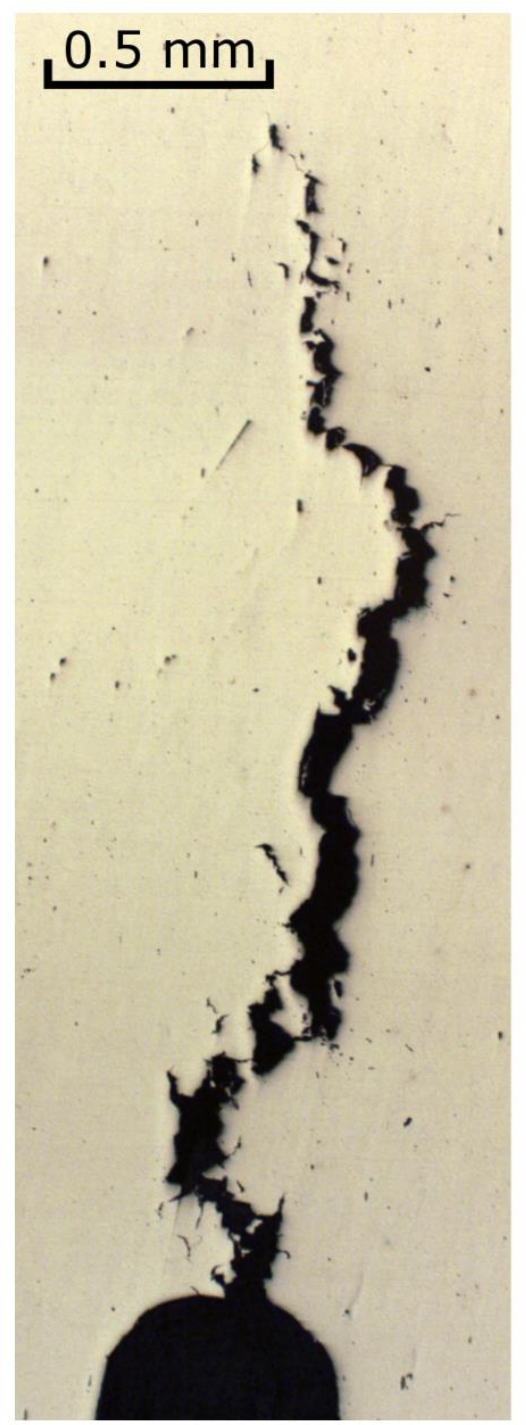

(a)

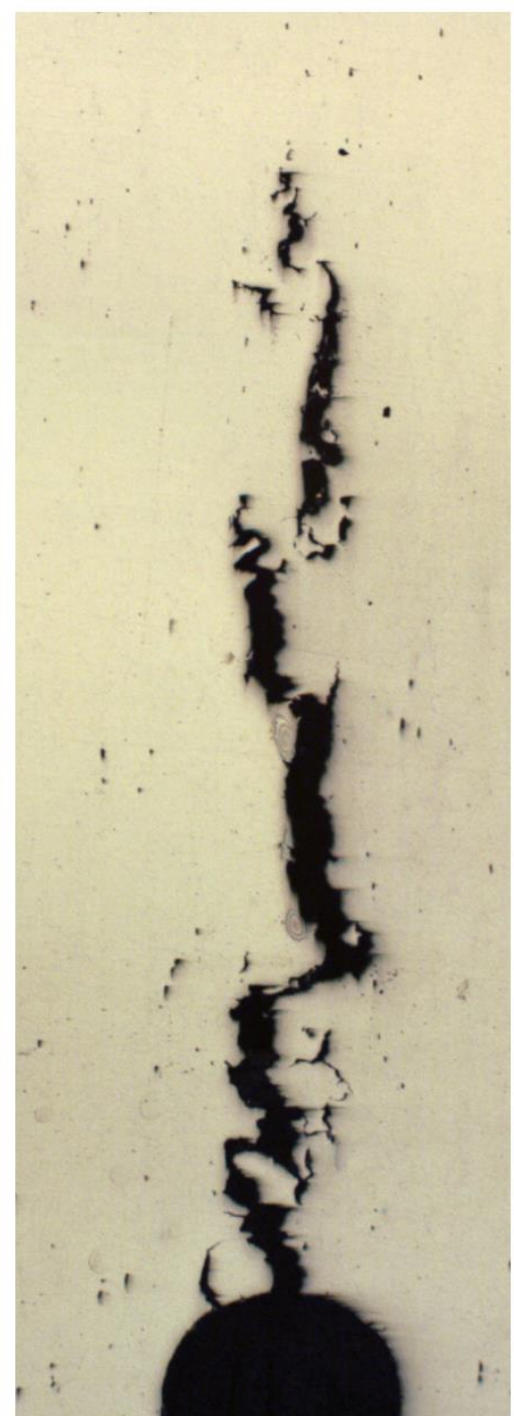

(b)

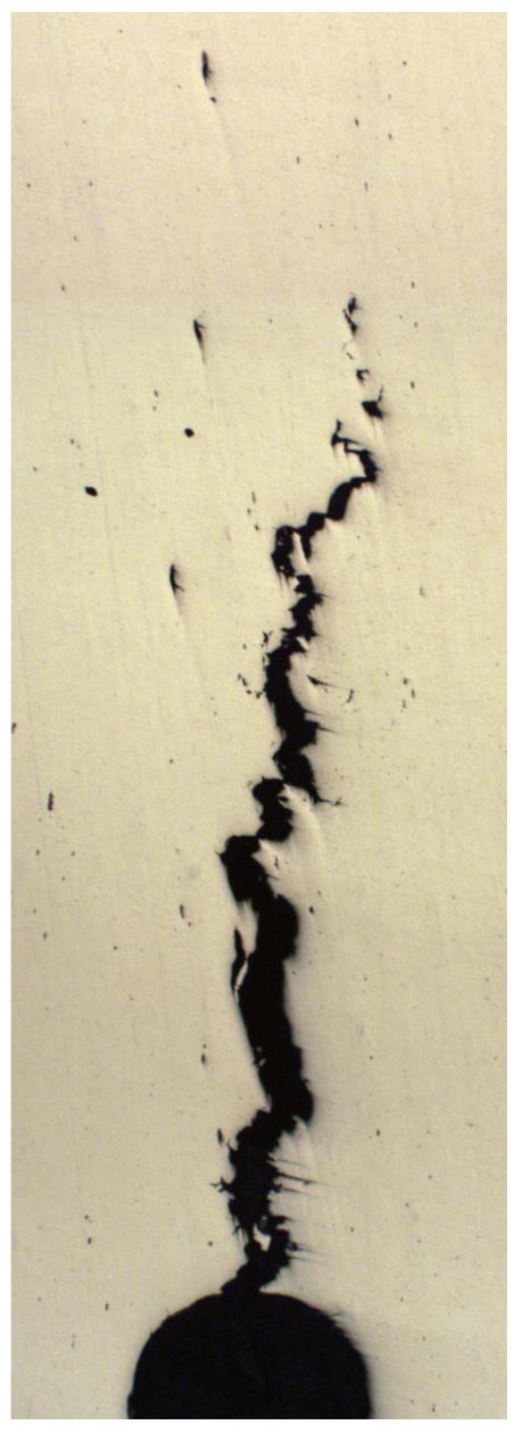

(c)

Figure 12 and 


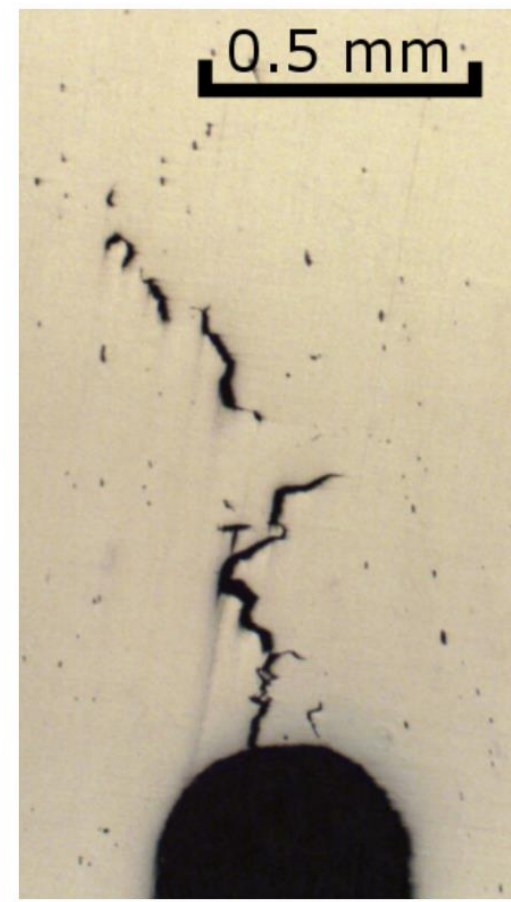

(a)

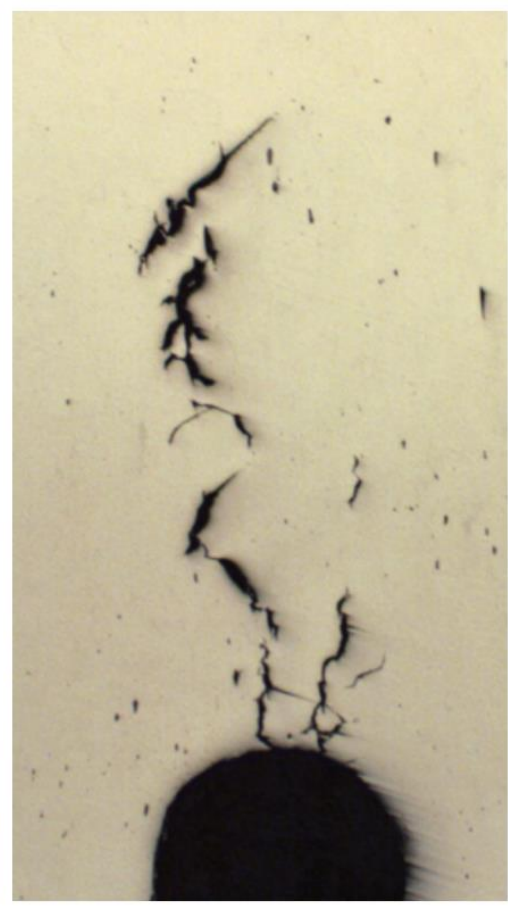

(b)

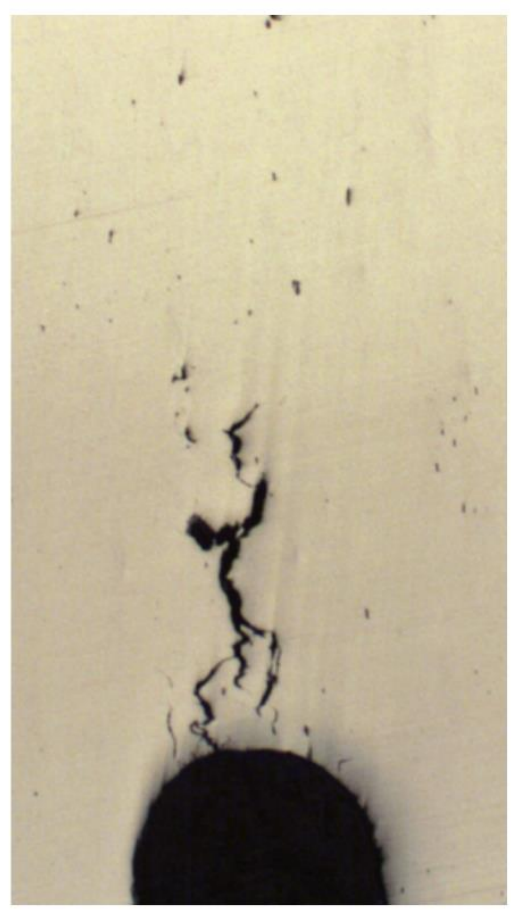

(c)

Figure 13 respectively. This crack growth is severely discontinuous (particularly for specimen CCG316_CT02) and non-planar, which is typical of creep. Also, despite the $20 \%$ side grooves some crack tunnelling is apparent. For both of these tests a definite increase in gradient on a plot of resistance vs. LLD is observed (see 

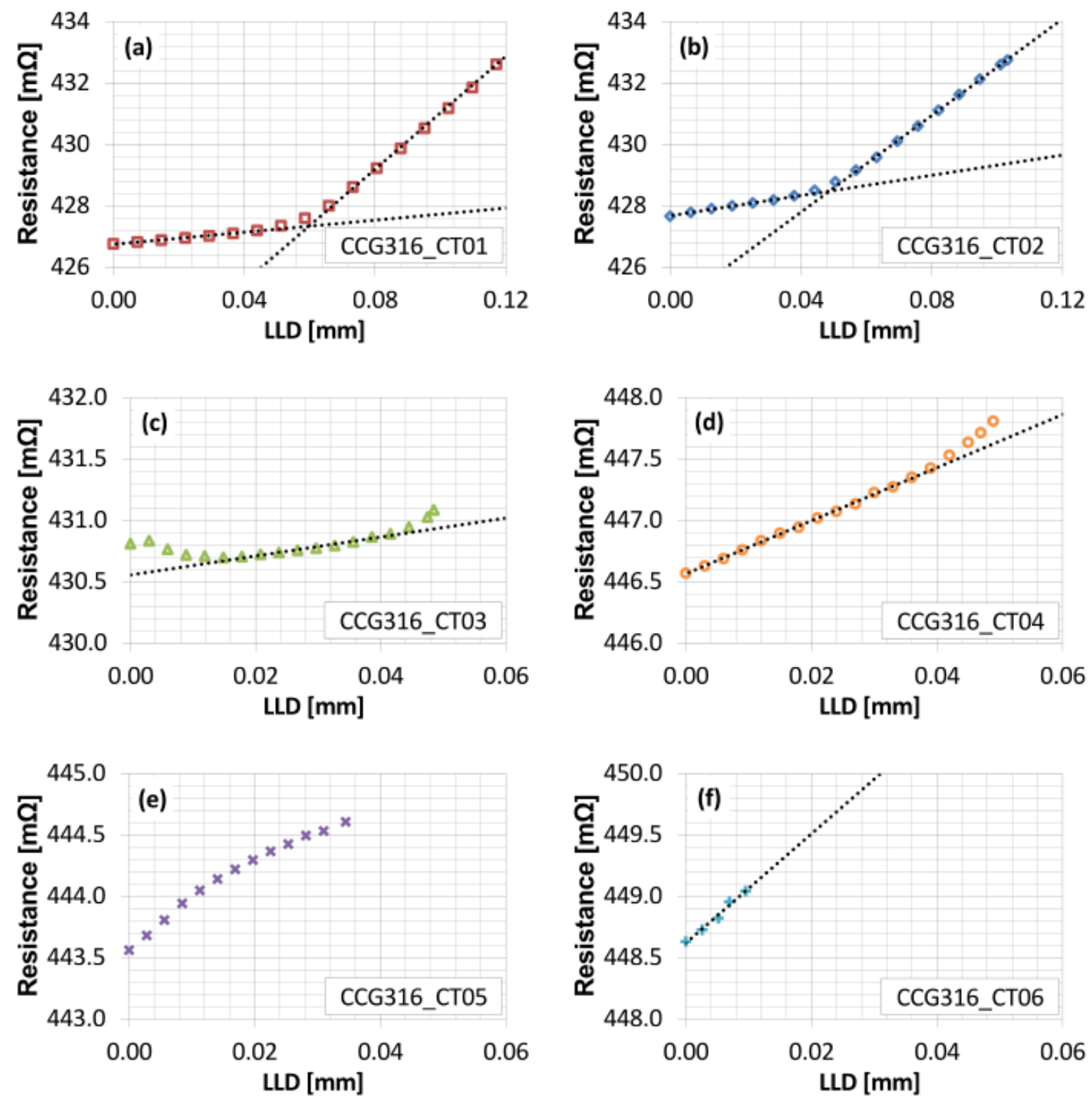

Figure 11(a) and (b) respectively).

For specimens CCG316_CT03 and CCG316_CT04 significant damage ahead of the EDM pre-crack was observed but this was only connected to the pre-crack close to the mid-plane of the specimen as observed in 


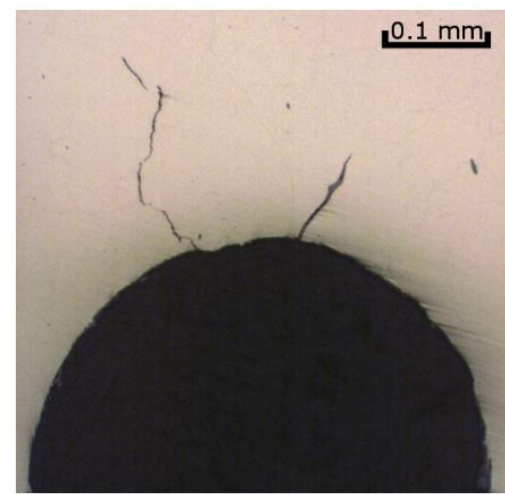

(a)

Figure 14 and

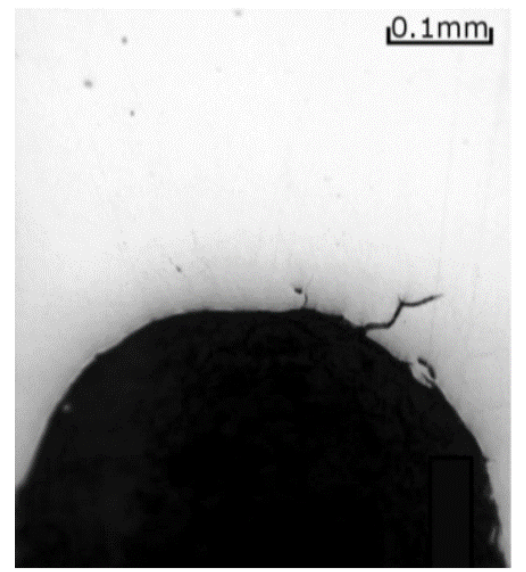

(a)

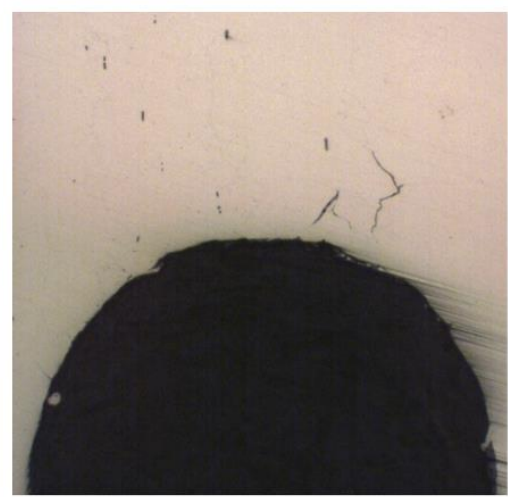

(b)

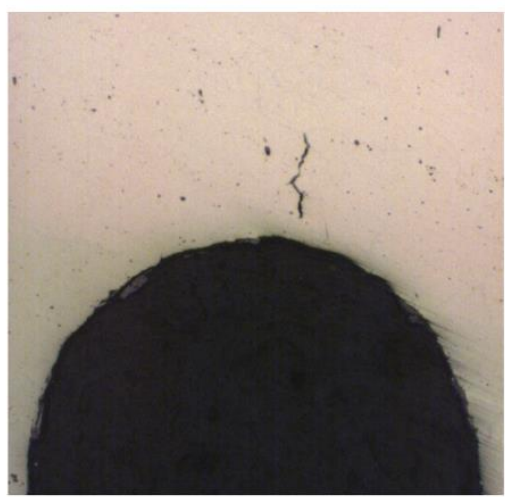

(c)

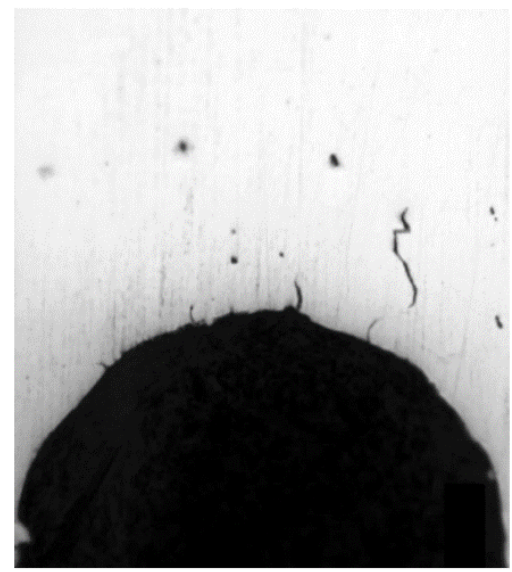

(b)

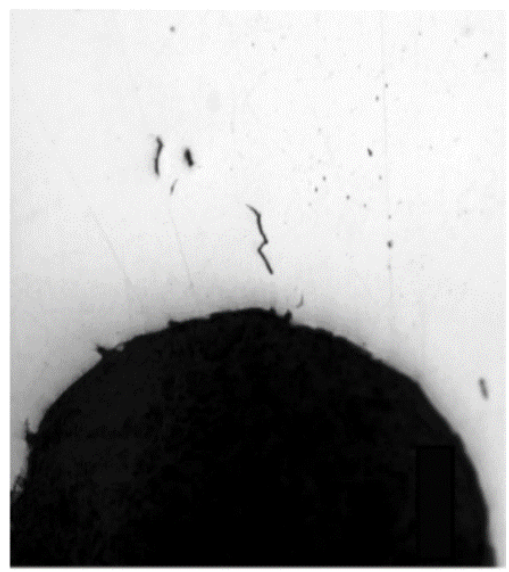

(c)

Figure 15 respectively. Both of these tests were terminated shortly after an increase in gradient on a plot of resistance vs. LLD (see 

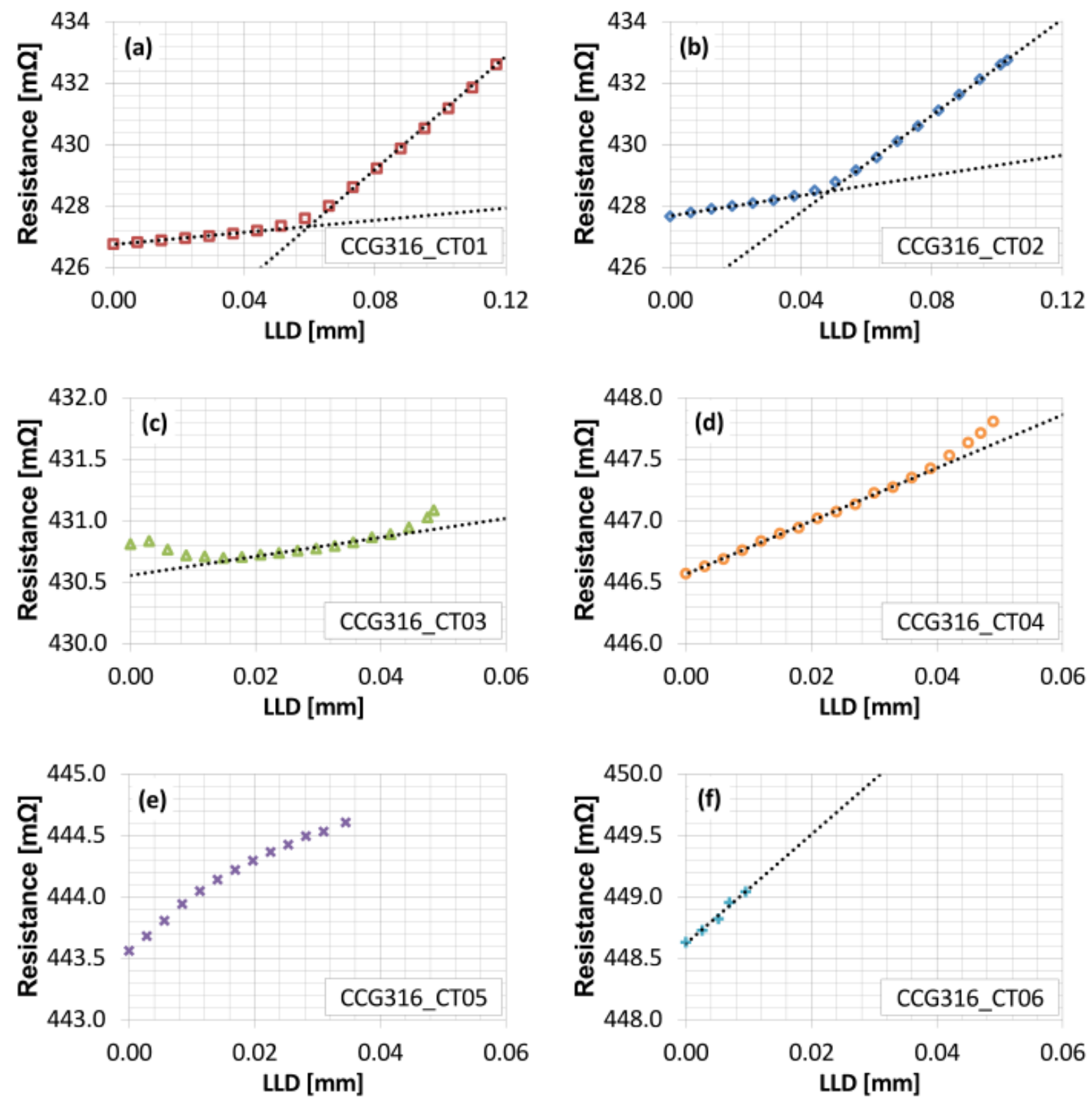

Figure 11(c) and (d) respectively). For specimens CCG316_CT05 and CCG316_CT06 there is no evidence of crack growth in

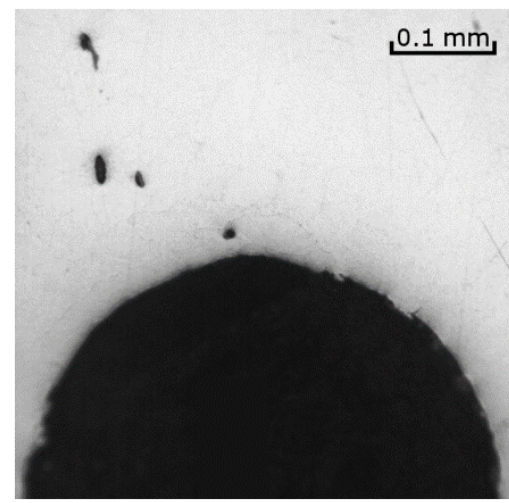

(a)

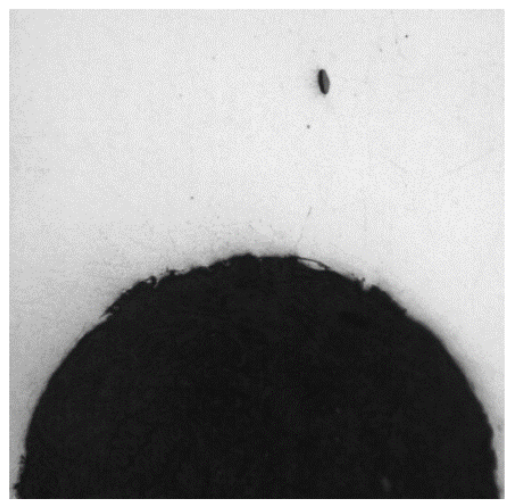

(b)

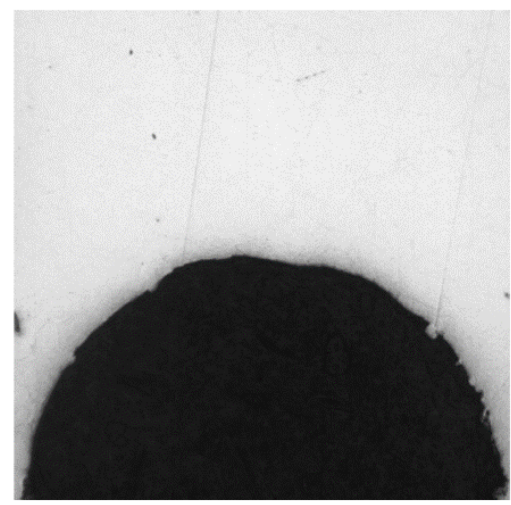

(c) 
Figure

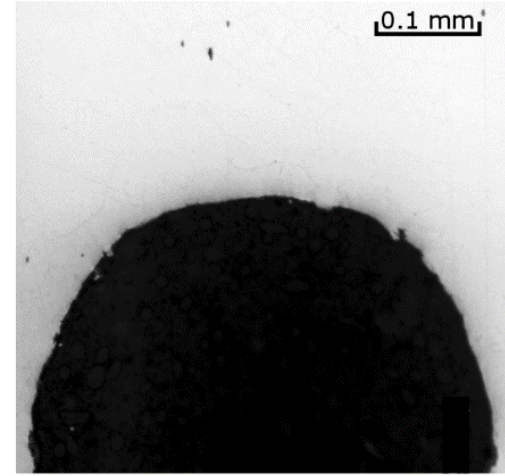

(a)
16

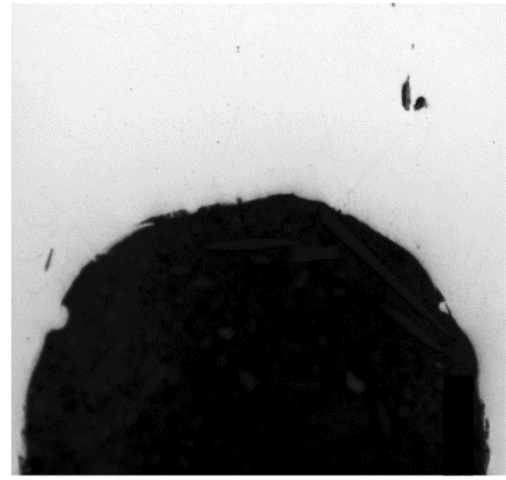

(b)

and

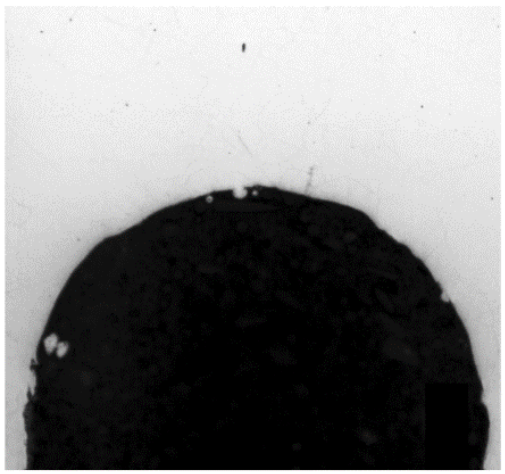

(c)

Figure 17 respectively, although there is some damage ahead of the pre-crack. Both of these tests were terminated prior to any increase in gradient on a plot of resistance vs. LLD (see 

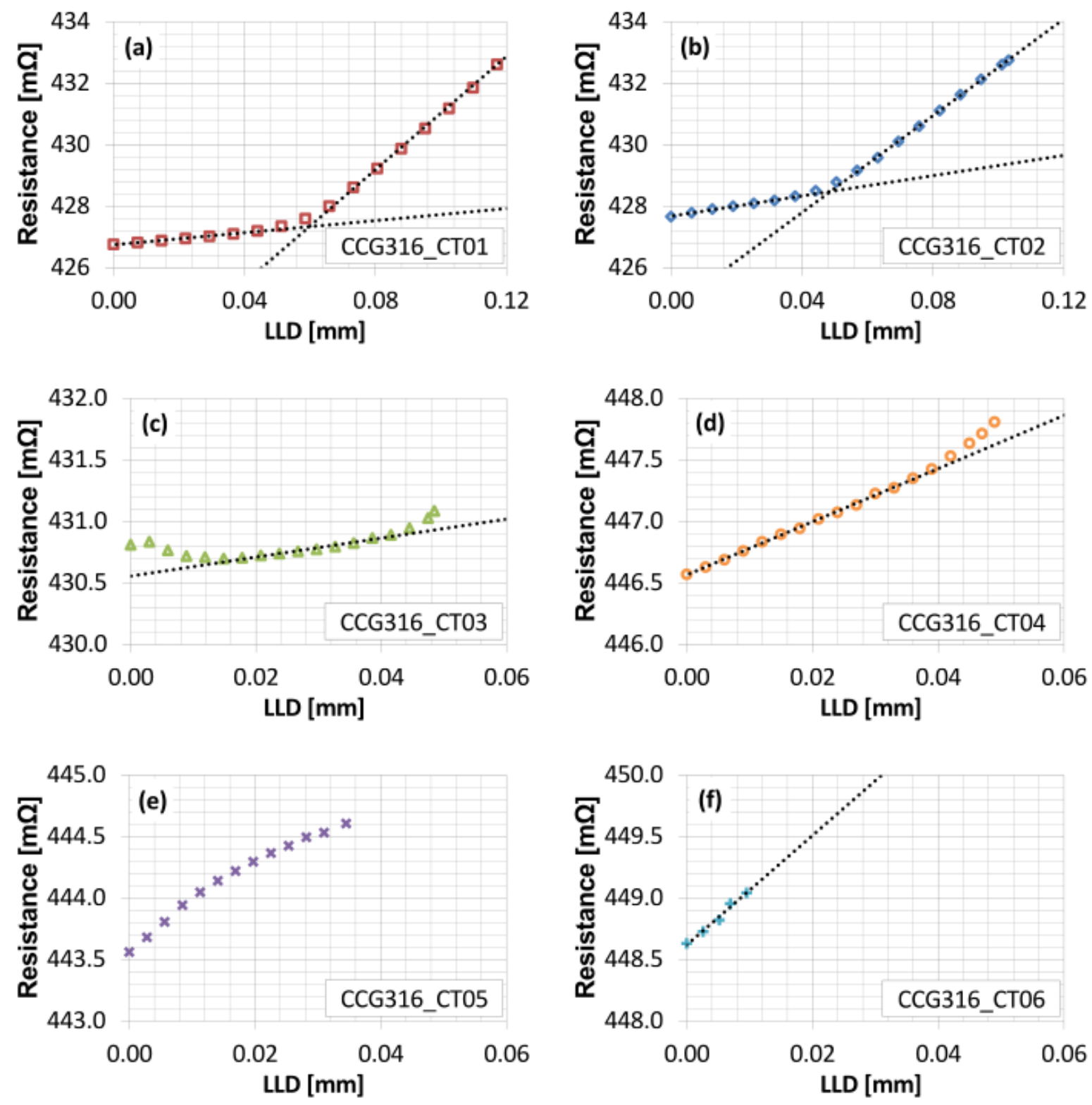

Figure 11(e) and (f) respectively). 


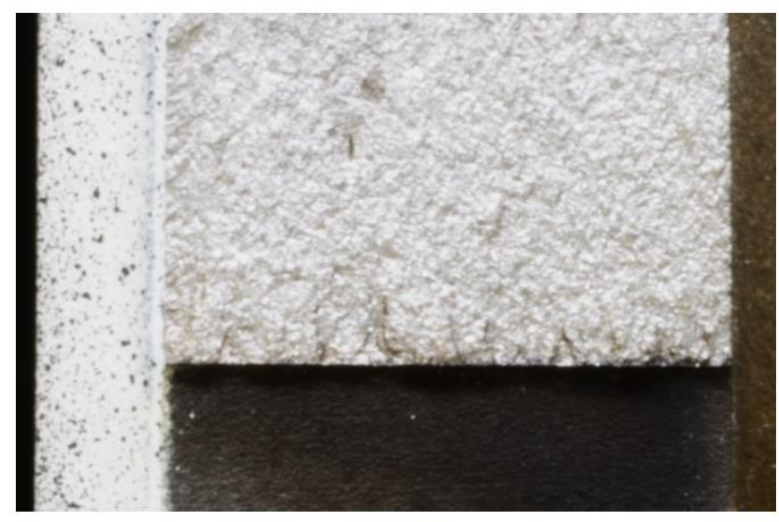

(a)

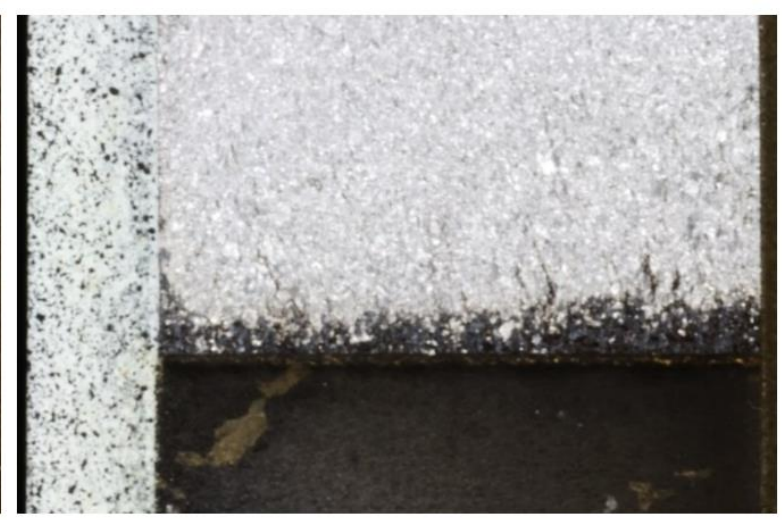

(b)

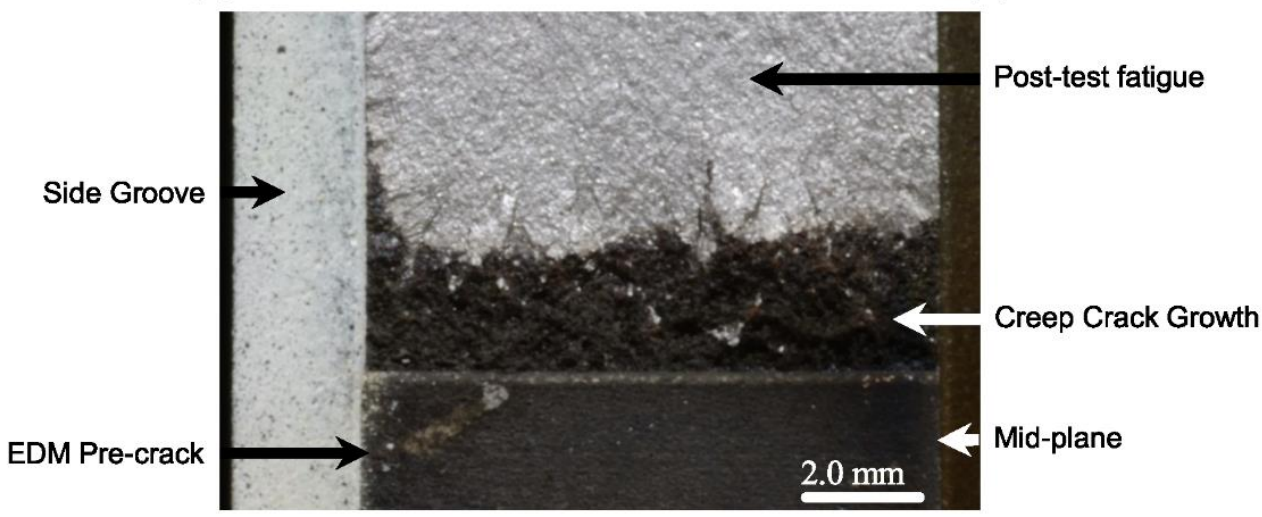

(c)

Figure 18 shows the fracture surface for specimens CCG316_CT01, CCG316_CT02 and CCG316_CT03. The fracture surface of specimen CCG316_CT03 is representative of specimen CCG316_CT04. The fracture surface of specimens CCG316_CT05 and CCG316_CT06 are not shown because no crack growth was observed. The mid-plane of the specimen is on the right-hand side of each image, and the side-groove is on the left. The dark, oxidised region ahead of the EDM pre-crack corresponds to CCG. This region contains small areas which are not oxidised. They are most prevalent on specimen CCG316_CT02 which is the specimen most significantly affected by discontinuous cracking (see 


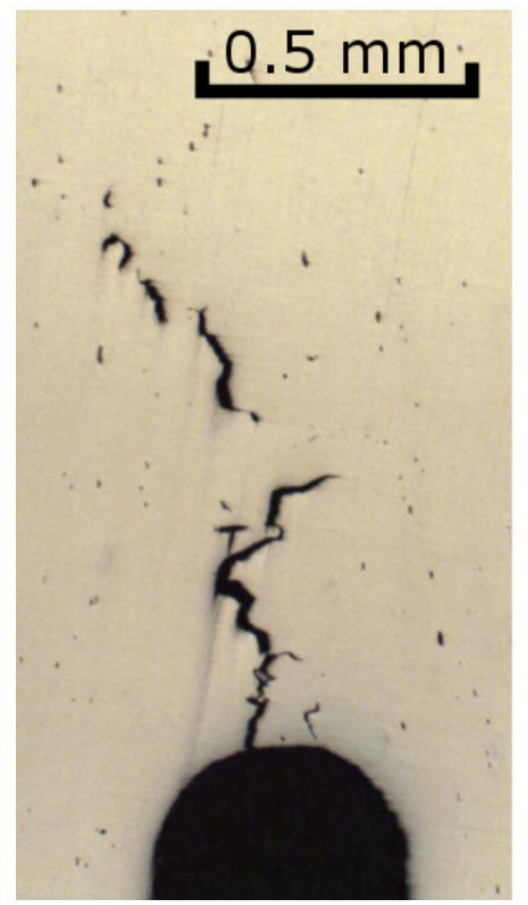

(a)

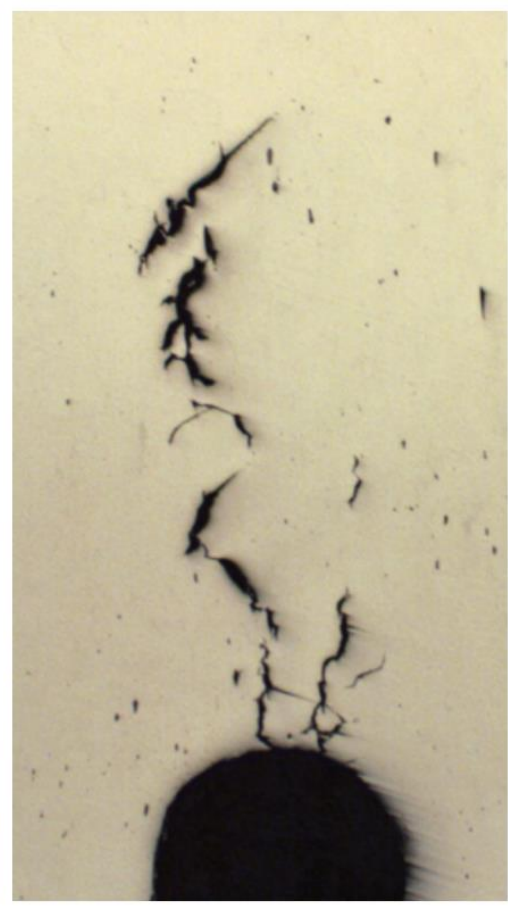

(b)

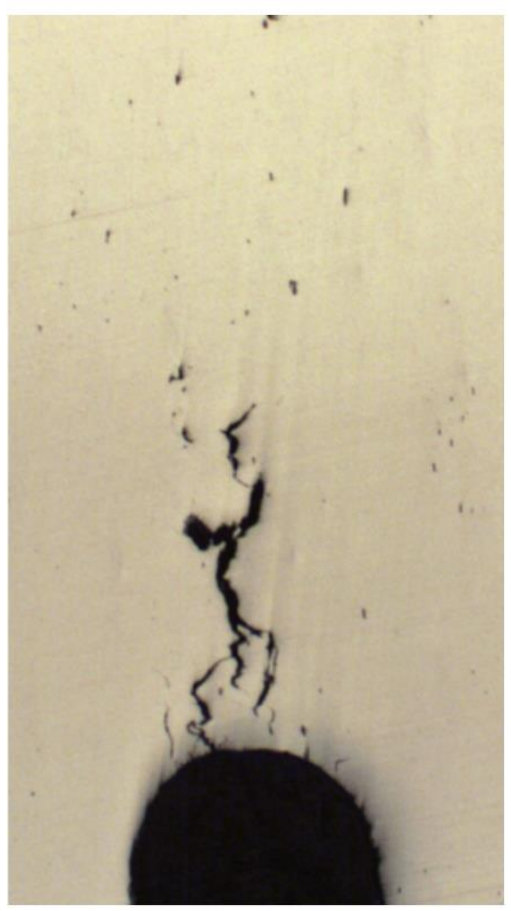

(c)

Figure 13) and are likely to correspond to islands of uncracked material which did not oxidise during the test. It also is possible that some of these regions are caused by contact with the opposing face when fatiguing the specimen open but a positive R-ratio (0.1) was used during this process to try to avoid this.

The average crack extension across the net thickness of the specimen, $\Delta a_{f}$, was calculated from the area of the crack. This was measured from the fracture surface using image processing software, Image J (NIH, 2013) and is provided in Table 3. The crack extension for specimens CCG316_CT03 and CCG316_CT04 is from a small, localised region close to the mid-plane of the specimen which is consistent with observations from the crack profiles.

\begin{tabular}{cc}
\hline Specimen & $\begin{array}{c}\Delta \boldsymbol{a}_{\boldsymbol{f}} \\
{[\mathbf{m m}]}\end{array}$ \\
\hline CCG316_CT01 & 2.44 \\
CCG316_CT02 & 0.62 \\
CCG316_CT03 & 0.01 \\
CCG316_CT04 & 0.01 \\
CCG316_CT05 & 0.00 \\
CCG316_CT06 & 0.00 \\
\hline
\end{tabular}

Table 3: Post-test crack length measurements. 


\subsubsection{Interrupted Tests Discussion}

\subsubsection{Interpretation of the PD Data}

To predict crack extension from a calibration function, such as the one provided in Equation (3), a normalising resistance value, $R_{0}$, is required. This value, as defined in the current method in ASTM E1457-15, corresponds to the minimum resistance measured throughout the test which, for all specimens tested here, is the initial resistance except for specimen CCG316_CT03 where a drop in resistance was observed at the start of the test. This value, denoted $R_{0}^{A S T M}$, is provided in Table 4 for each of the six tests. If however the increase in gradient on a plot of resistance (or PD) against LLD corresponds to the onset of crack growth, the resistance at this point would be a more appropriate value. This would remove any change in resistance due to strain which occurs prior to crack initiation from the calculation of crack growth and is analogous to the method proposed by Lowes and Fearnehough for fracture toughness testing. This value, denoted $R_{0}^{M O D}$, is provided in Table 4 for the four tests which were interrupted after this increase in gradient was observed.

\begin{tabular}{ccc}
\hline Specimen ID & $\begin{array}{c}\boldsymbol{R}_{\mathbf{0}}^{A S T M} \\
{[\mathbf{m} \boldsymbol{\Omega}]}\end{array}$ & $\begin{array}{c}\boldsymbol{R}_{\mathbf{0}}^{\text {MOD }} \\
{[\mathbf{m} \boldsymbol{\Omega}]}\end{array}$ \\
\hline CCG316_CT01 & 426.8 & 427.3 \\
CCG316_CT02 & 427.7 & 428.5 \\
CCG316_CT03 & 430.7 & 430.9 \\
CCG316_CT04 & 446.6 & 447.4 \\
CCG316_CT05 & 443.6 & - \\
CCG316_CT06 & 448.0 & - \\
\hline
\end{tabular}

Table 4: Key resistance measurements obtained from the interrupted CCG tests

\subsubsection{Measuring Creep Crack Initiation}

The increase in gradient on a plot of resistance (or PD) against LLD appears to correspond to the point at which damage first links up with the pre-crack. This is demonstrated by observations from the crack profiles and fracture surfaces. For specimens CCG316_CT03 and CCG316_CT04, which were interrupted immediately after the increase in gradient, significant damage is observed in the form of micro-cracks, but they are only linked to the EDM pre-crack close to the mid-plane. This region of high constraint is the most likely location for crack initiation to occur which suggests that the test was interrupted shortly after the onset of crack growth. Conversely, no crack growth was observed for specimens CCG316_CT05 and 
CCG316_CT06 which did not demonstrate an increase in gradient, despite a notable increase resistance.

Various definitions of the initiation time are summarised in Table 5. The onset of crack growth based on the method in ASTM E1457, $t_{0}^{A S T M}$, is the time corresponding to $R_{0}^{A S T M}$ provided in Table 4 i.e. the time at which the minimum resistance measurement is recorded throughout the test. The onset of crack growth based on the modified method, $t_{0}^{M O D}$, is the time corresponding to $R_{0}^{M O D}$ provided in Table 4 i.e. the time at which the gradient on a plot of PD vs. LLD increases. The corresponding times for $0.2 \mathrm{~mm}$ of crack growth (the 'engineering definition' of crack initiation) are denoted $t_{0.2}^{A S T M}$ and $t_{0.2}^{M O D}$ respectively. These are calculated based on crack length predictions obtained from the calibration function in Equation (3) and corrected based on the average crack extension measurements in Table 3 using Equation (2). Initiation times are not provided for specimens CCG316_CT05 and CCG316_CT06 because no crack extension was observed.

\begin{tabular}{ccccc}
\hline \multirow{2}{*}{ Specimen ID } & $\begin{array}{c}t_{0}^{\text {ASTM }} \\
{[\mathbf{h}]}\end{array}$ & $\begin{array}{c}t_{0}^{\text {MOD }} \\
{[\mathbf{h}]}\end{array}$ & $\begin{array}{c}t_{0.2}^{\text {ASTM }} \\
{[\mathbf{h}]}\end{array}$ & $\begin{array}{c}t_{0.2}^{\text {MOD }} \\
{[\mathbf{h}]}\end{array}$ \\
\hline CCG316_CT01 & 0 & 237 & 450 & 478 \\
CCG316_CT02 & 0 & 226 & 396 & 463 \\
CCG316_CT03 & 8 & 94 & - & - \\
CCG316_CT04 & 0 & 82 & - & - \\
\hline
\end{tabular}

Table 5: Comparison of initiation times

There is a significant difference in the times corresponding to the onset of crack growth measured by the two methods. The ASTM method assumes the onset of crack growth occurs at the start of the test (unless there is an initial drop in PD) whilst the modified method predicts the onset of crack growth after 200 hours for tests CCG316_CT01 and CCG316_CT02 and approximately half this time for tests CCG316_CT03 and CCG316_CT04. This scatter is typical of creep crack initiation data.

Despite the large discrepancy in the times corresponding to the onset of crack growth for the two methods, the times for $0.2 \mathrm{~mm}$ of crack extension to occur are relatively similar. The modified method predicts it takes 6\% longer than the ASTM method for specimen CCG316_CT01 and 17\% longer for specimen CCG316_CT02. Although these differences are relatively small, this is not always the case. For example, there was more than a factor of 5 difference in the preliminary test performed on P91. This is because of the higher creep 
ductility of P91. Assuming crack initiation occurs when this ductility is exhausted, the PD system will measure larger spurious crack extensions due to creep in a P91 specimen. This can be observed qualitatively by comparing the diameter of the EDM pre-crack measured from the post-test crack profiles. For the Type $316 \mathrm{H}$ specimen it is $\sim 0.45 \mathrm{~mm}$, as measured from

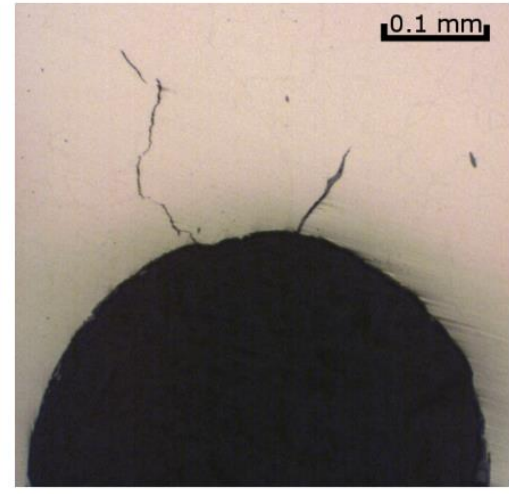

(a)

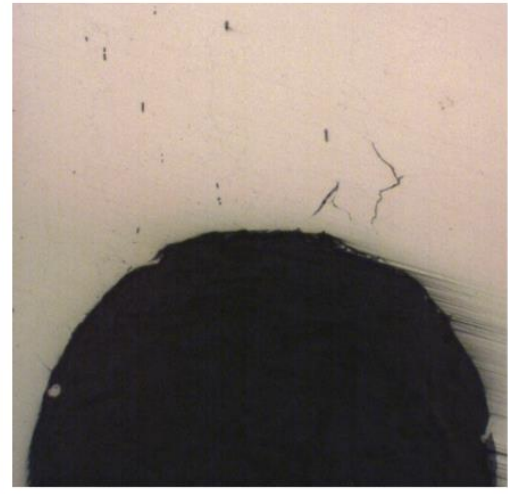

(b)

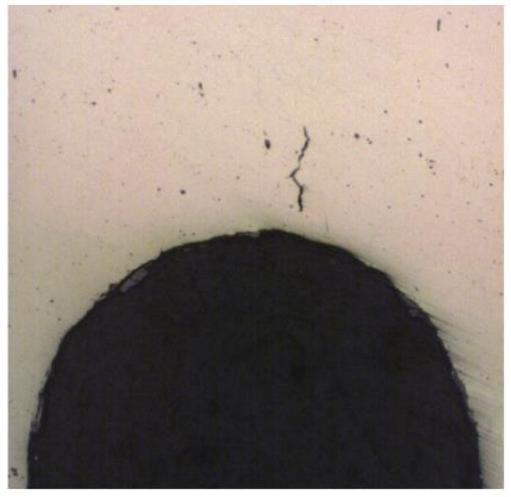

(c)

Figure 14, and for the P91 specimen it is $\sim 0.60 \mathrm{~mm}$, as measured from

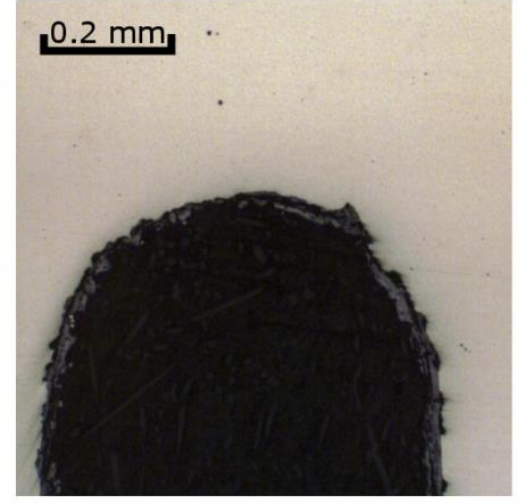

(a)

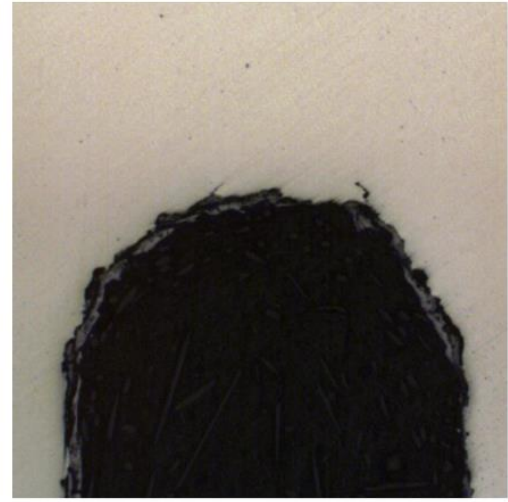

(b)

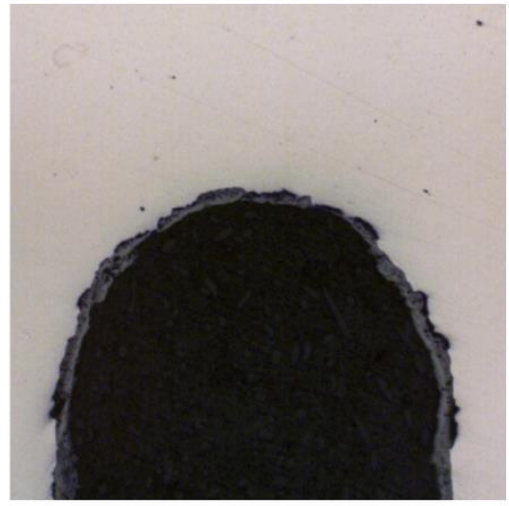

(c)

Figure 7. Both specimens initially had nominally identical EDM pre-cracks so the total inelastic strain (plastic + creep) at the crack tip is much larger in the P91 specimen despite smaller plastic strains, as demonstrated by the much lower $\sigma_{r e f} / \sigma_{0.2}$ ratio (which is 0.39 compared with 0.99 for the Type $316 \mathrm{H}$ specimen).

\subsubsection{Measuring Creep Crack Growth}

The crack extension measured from the post-test fracture surfaces are compared with the values predicted from the PD technique in Table 6. The predicted final crack growth based on the method in ASTM E1457-15, $\Delta a_{p f}^{A S T M}$, is calculated using the calibration function in Equation (3) using the minimum resistance measurement $\left(R_{0}^{A S T M}\right.$ in Table 4). The predicted crack growth based on the modified method, $\Delta a_{p f}^{M O D}$, is calculated using the same calibration function 
but using the resistance corresponding to the increase in gradient on a plot of resistance vs. $\operatorname{LLD}\left(R_{0}^{M O D}\right.$ in Table 4).

\begin{tabular}{cccc}
\hline Specimen & $\begin{array}{c}\Delta \boldsymbol{a}_{\boldsymbol{f}} \\
{[\mathbf{m m}]}\end{array}$ & $\begin{array}{c}\Delta \boldsymbol{a}_{\boldsymbol{p f}}^{\boldsymbol{A S T M}} \\
{[\mathbf{m m}]}\end{array}$ & $\begin{array}{c}\Delta \boldsymbol{a}_{\boldsymbol{p f}}^{\boldsymbol{M O D}} \\
{[\mathbf{m m}]}\end{array}$ \\
\hline CCG316_CT01 & 2.44 & 1.32 & 1.30 \\
CCG316_CT02 & 0.62 & 0.23 & 0.20 \\
CCG316_CT03 & 0.01 & 0.02 & 0.01 \\
CCG316_CT04 & 0.01 & 0.05 & 0.02 \\
CCG316_CT05 & 0.00 & 0.05 & 0.00 \\
CCG316_CT06 & 0.00 & 0.02 & 0.00 \\
\hline
\end{tabular}

Table 6: Comparison of the final crack length predicted from the PD technique with measurements from the post-test fracture surface.

For tests terminated before or immediately after crack initiation (CCG316_CT03 to CCG316_CT06), the crack extension predicted using the modified method of interpreting the PD data is in good agreement with the fracture surface observations. The crack extension predicted using the ASTM method however, consistently overestimates the crack growth. The likely cause of this discrepancy is creep strain. For specimens CCG316_CT03 to CCG316_CT06 the spurious crack extension due to strain is $<0.05 \mathrm{~mm}$ however, as identified from the preliminary test on P91, for materials with high creep ductility this can be $>0.35 \mathrm{~mm}$. For tests terminated after significant crack growth (CCG316_CT01 and CCG316_CT02) the PD technique underestimates the crack extension compared to fracture surface observations. This is independent of the method of interpreting the PD data which has little influence on the final crack length predictions. For specimen CCG316_CT01 the PD predicts approximately half the crack extension measured from the fracture surface and for specimen CCG316_CT02 this reduces to a third. The most likely source of these discrepancies is the non-ideal crack morphology observed in 


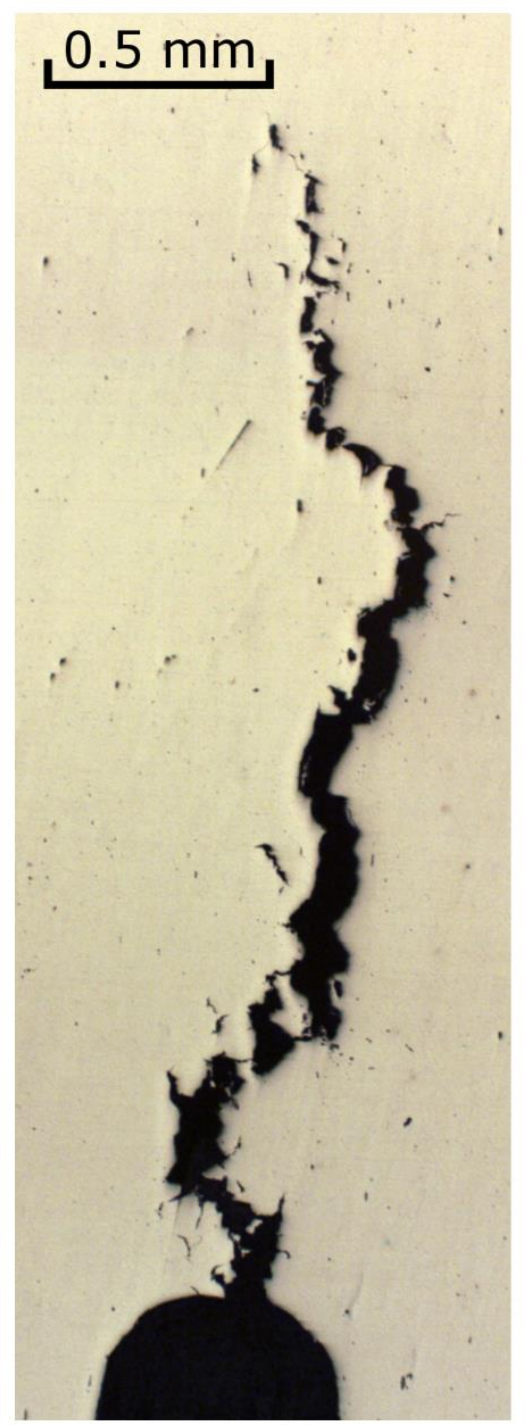

(a)

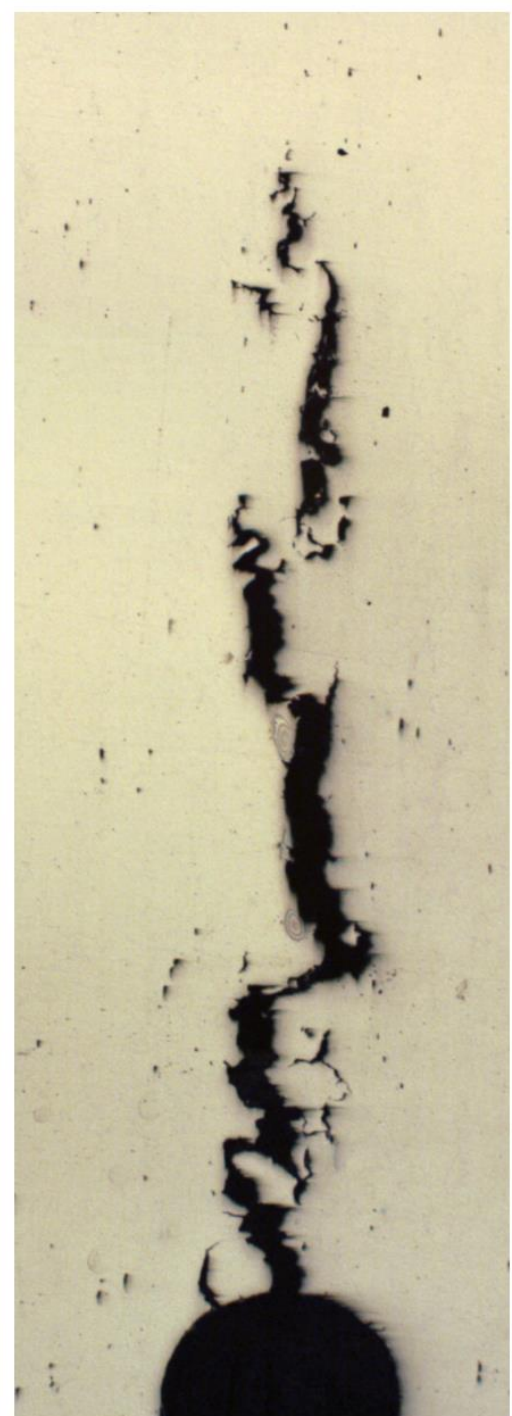

(b)

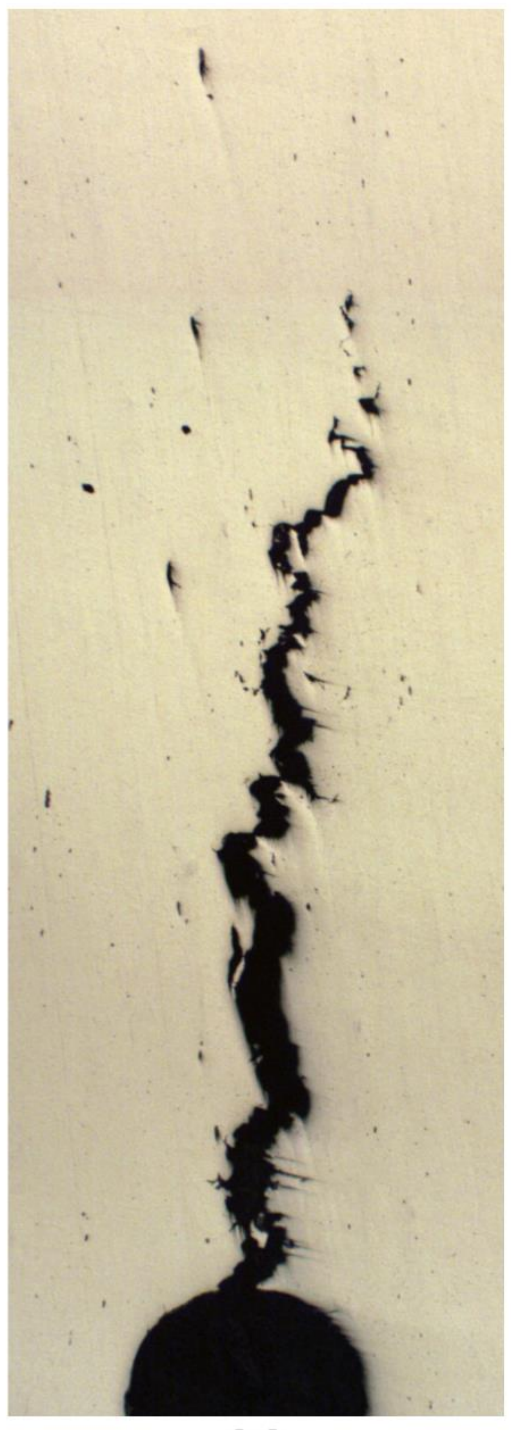

(c)

Figure 12 and 


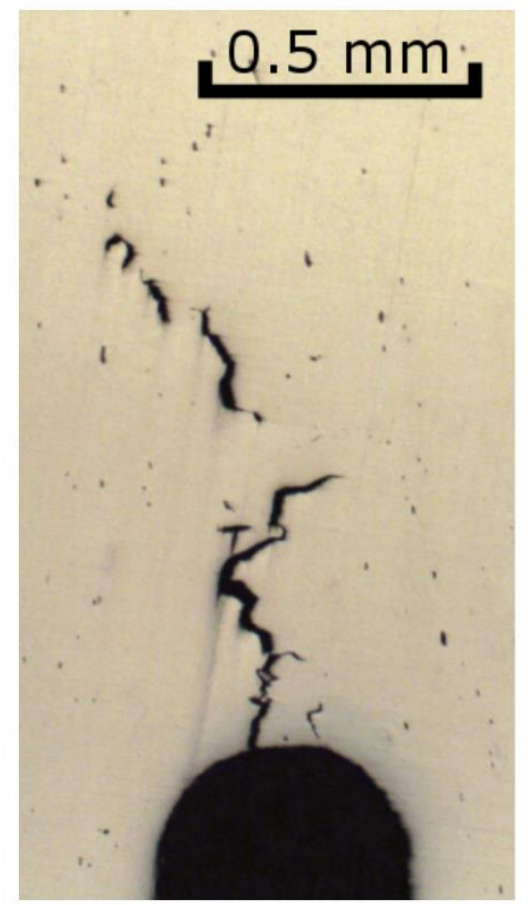

(a)

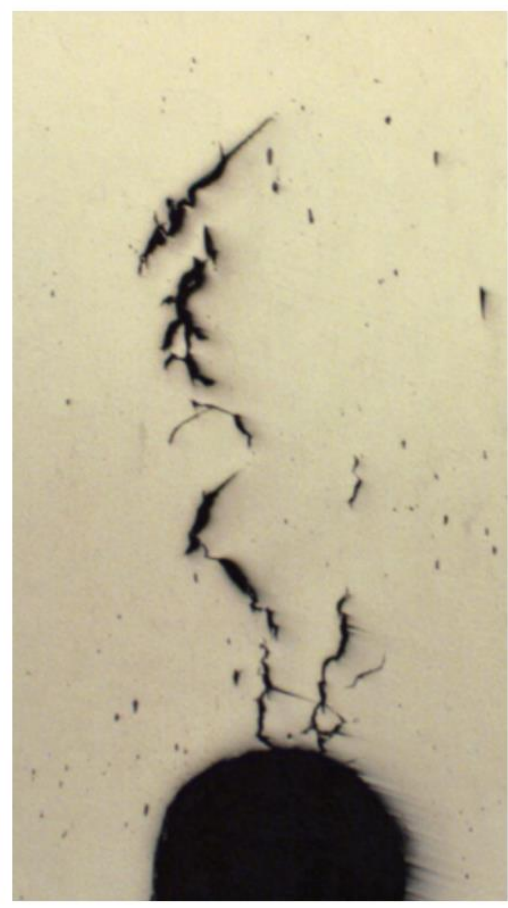

(b)

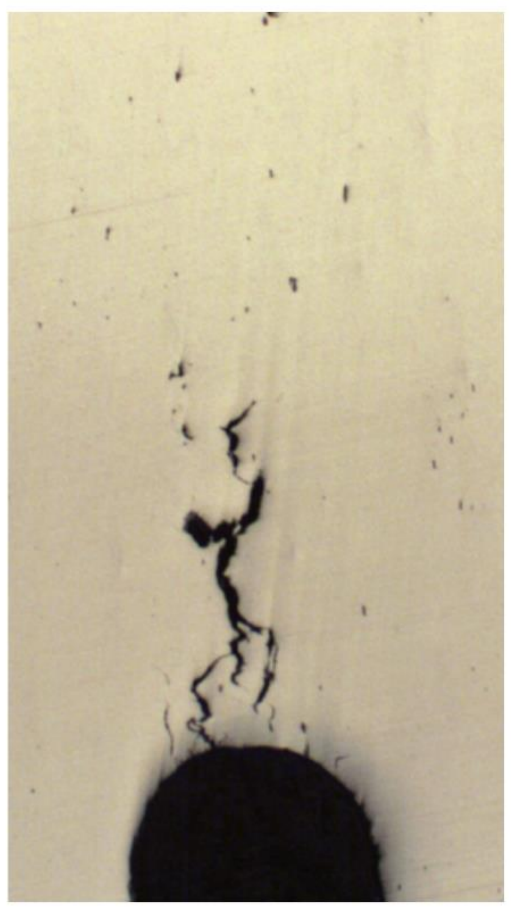

(c)

Figure 13, particularly the discontinuous cracking and asperities on the fracture surface. This prevents clean separation of the crack faces, providing alternative paths for the current and resulting in an underestimation of the crack growth. Strain is unlikely to account for this discrepancy because this tends to increase rather than reduce the PD measurement.

ASTM E1457-15 allows a maximum discrepancy of 15\% between the crack growth predicted from the PD and observed from the fracture surface. Tests CCG316_CT01 and CCG316_CT02 are therefore invalid according to this criteria however, the crack morphologies demonstrated in are typical of creep crack growth which suggests that many tests will be rendered invalid based on this criteria. This highlights a fundamental limitation of the PD technique when applied to cracks with complex morphologies however, the static load requirement and hostile environment of a creep crack growth test preclude other measurement techniques. In the absence of a viable alternative, a pragmatic approach has been implemented here whereby all PD crack length measurements have been corrected based on the final crack length measured from the fracture surface using Equation (2). 

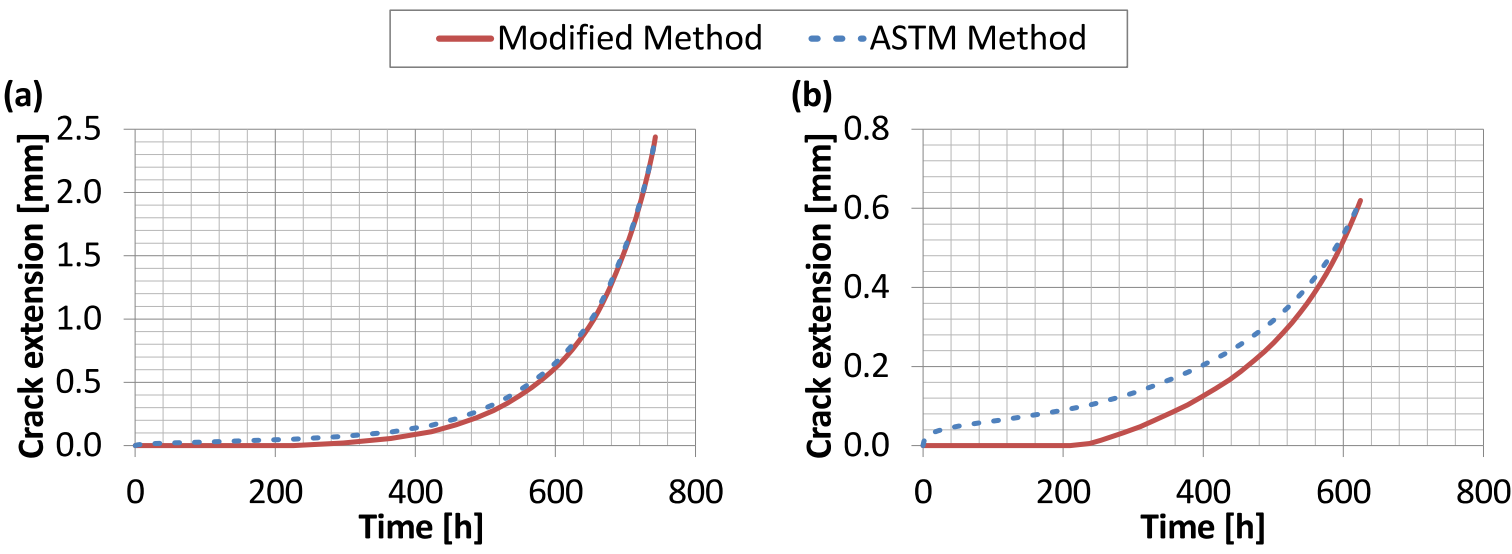

Figure 19 compares the corrected crack extension for the ASTM and modified methods of interpreting the PD data for specimens CCG316_CT01 and CCG316_CT02.

The ASTM method predicts immediate crack growth for both specimens, whilst the modified method demonstrates a period of incubation. The erroneous crack growth predicted by the ASTM method during this incubation period also introduces errors in the subsequent crack growth rates as shown by the different gradients for the two methods of interpreting the PD data in
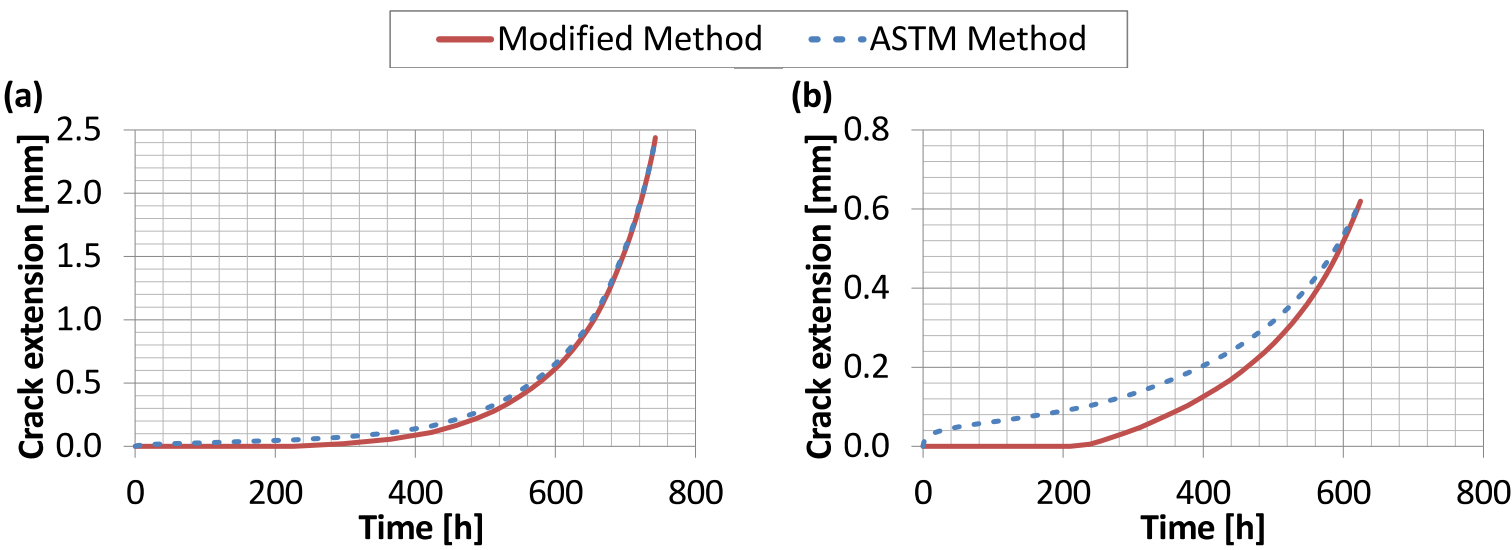

Figure 19. For specimen CCG316_CT01, the difference between the two methods is very small because the erroneous crack growth during incubation is only $0.05 \mathrm{~mm}$, which is much smaller than the actual crack growth that occurs during the test $(2.44 \mathrm{~mm})$. For specimen CCG316_CT02 however, the difference between the two methods is more apparent because the erroneous crack growth is $0.10 \mathrm{~mm}$ which is the same order of magnitude as the actual crack growth $(0.62 \mathrm{~mm})$. The crack growth rate is under-predicted by the ASTM method. This is non-conservative.

The differences in crack growth rate observed in 

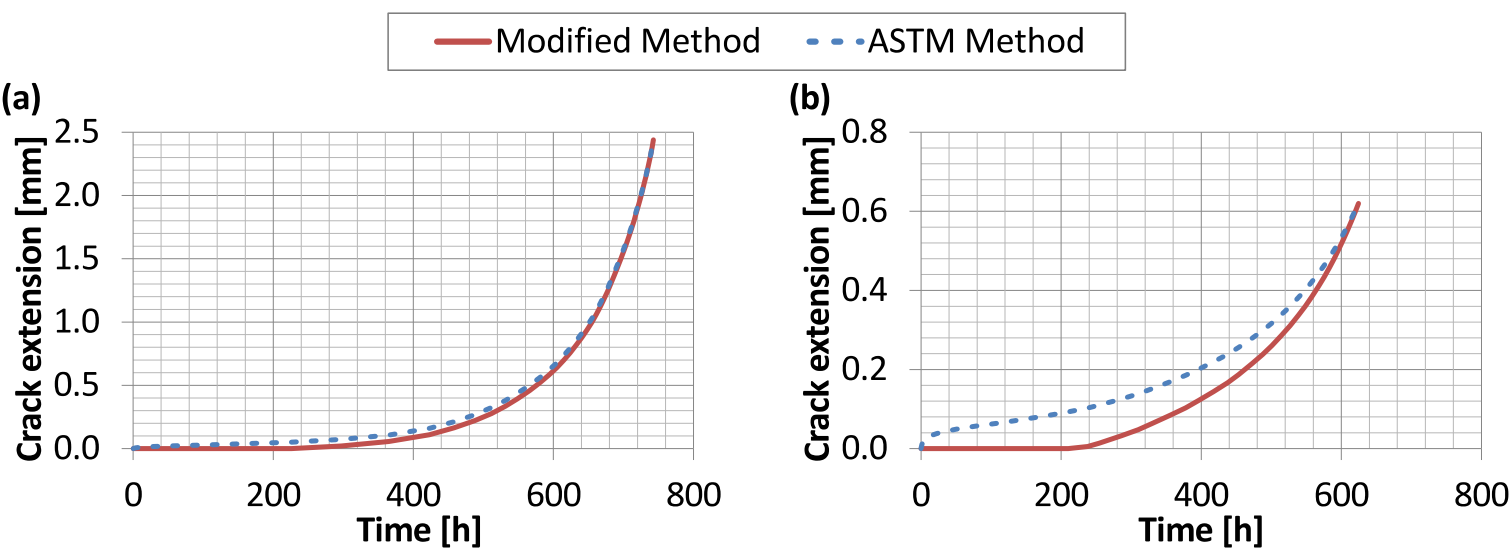

Figure 19 are caused by applying the crack length correction in Equation (2). If this correction is not applied then the two methods would predict almost identical crack growth rates after the incubation period but different final crack lengths however, as discussed above, this correction is necessary to account for the alternative current paths provided by discontinuous cracking and asperities on the crack faces which are typical of CCG. It is therefore prudent to apply this correction.

The crack growth rates for specimens CCG316_CT01 and CCG316_CT02, have been correlated with the crack tip parameter $C^{*}$ in

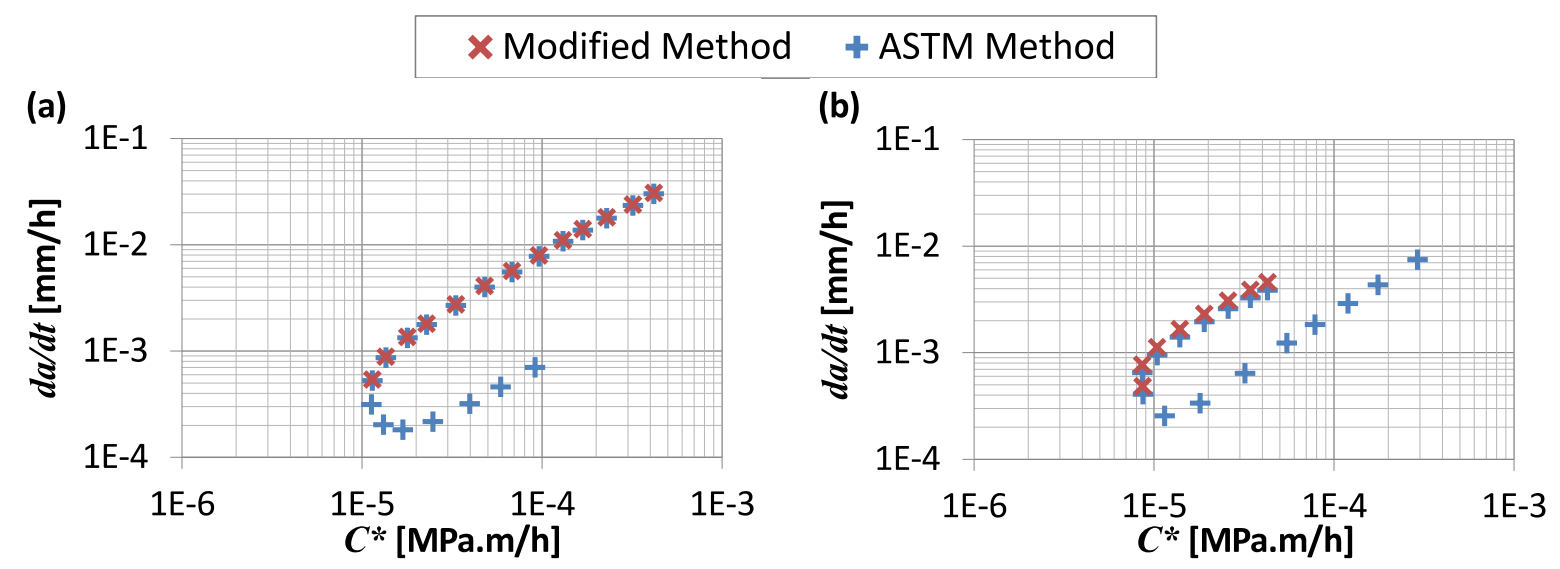

Figure 20(a) and 

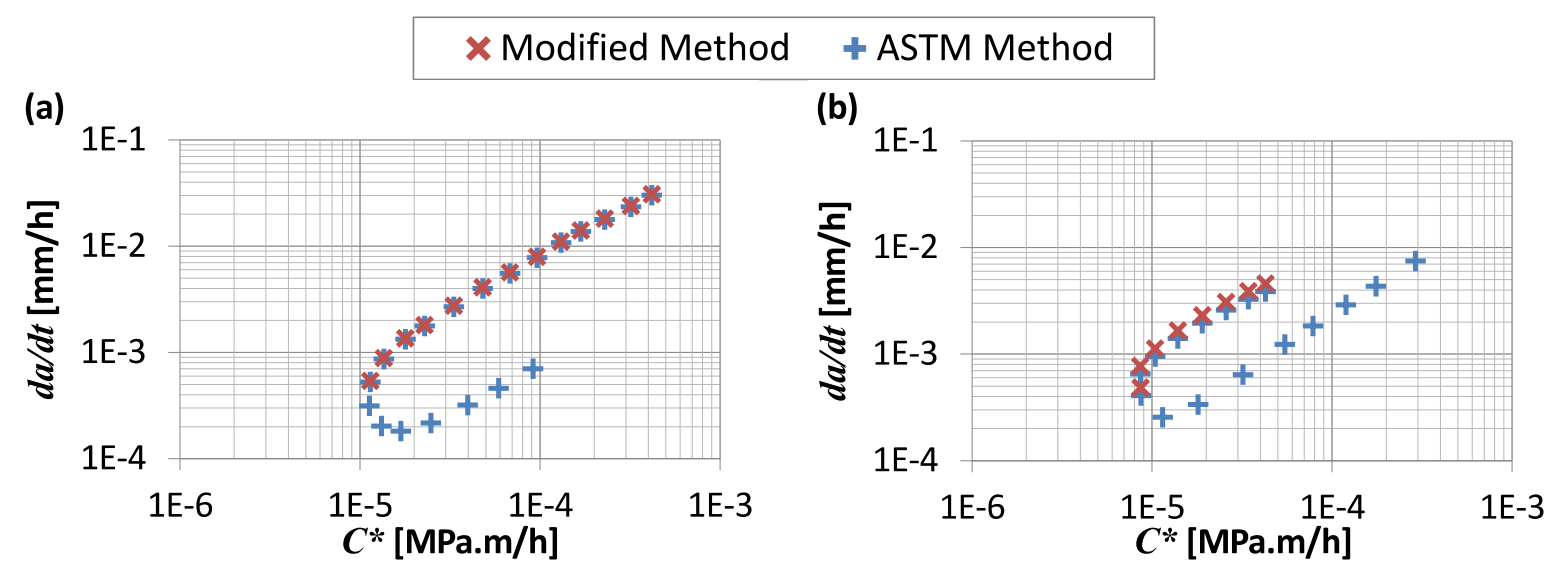

Figure 20(b) respectively. Both methods of analysing the PD data are shown. As expected, the difference between the steady-state crack growth rates predicted by the two methods is larger for specimen CCG316_CT02 but, on a log-log plot, this difference is small although larger differences would be expected if the actual crack extension is particularly small or the creep ductility of the material is particularly high. The main difference between the two methods of analysing the PD data observed in

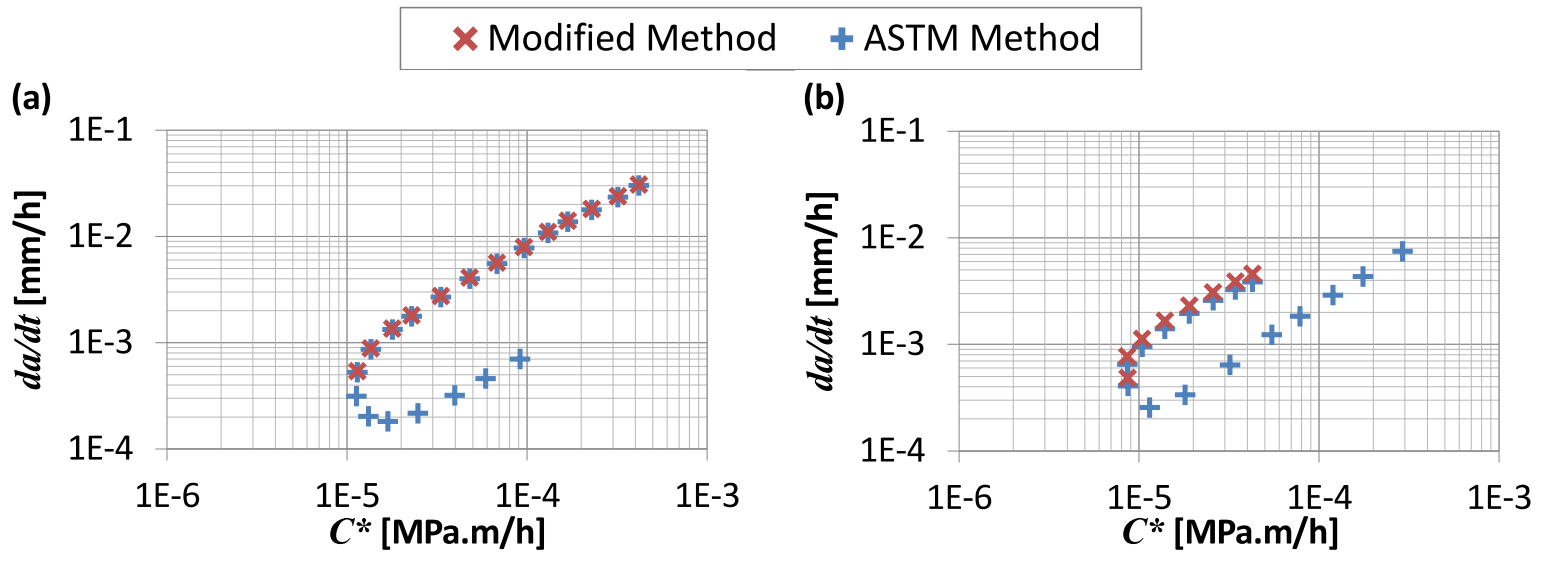

Figure 20 is in the so-called "tail" region. Only the ASTM method predicts a sizable tail which demonstrates that, for this material, the tail is caused by strains being erroneously interpreted as crack growth.

\section{4. $\quad$ Finite Element Validation}

To validate the proposed interpretation of PD data from a creep crack growth test a sequentially coupled structural-electrical FE model has been used. This modelling approach has been recently used to perform a similar validation for fracture toughness testing (Tarnowski et al., 2017). Here it will be used to confirm that the change in gradient on a plot of PD (or resistance) 
vs. LLD is due to the onset of crack growth and that the effects of strain during any subsequent crack growth are small.

\subsection{Finite Element Validation: Methodology}

\subsubsection{Geometry and Mesh}

Two 3D quarter models of the $\mathrm{C}(\mathrm{T})$ specimen were produced using Abaqus [11]. Model 'CCG_CI' was used to assess the influence of strain on resistance up to the point of crack initiation. Model 'CCG_CG' was used to assess the entire CCG test, including crack growth. The two models are shown in

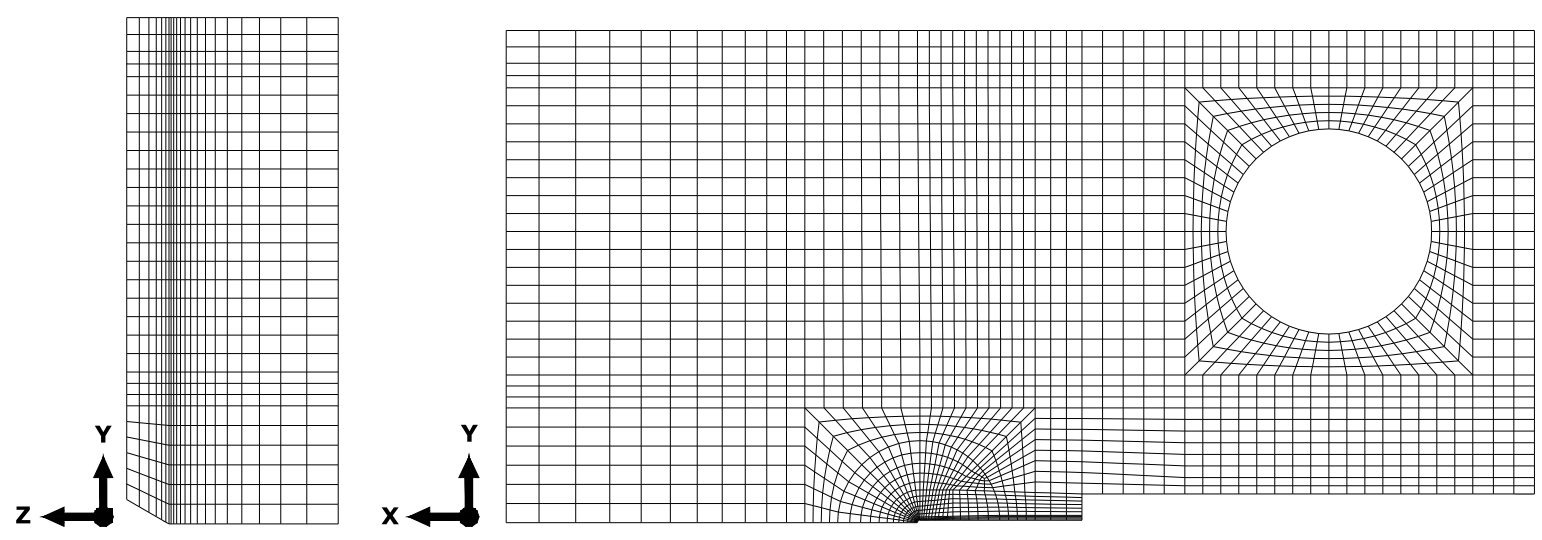

Figure 21 and
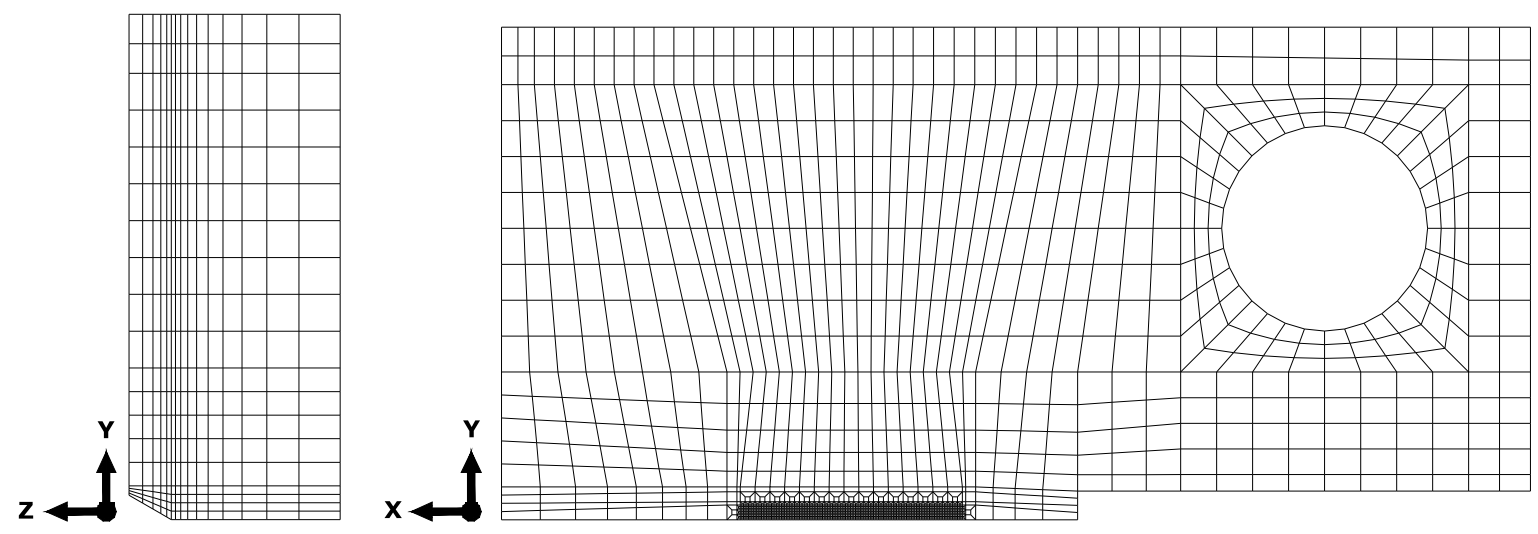

Figure 22 respectively. Model 'CCG_CI' includes the $0.15 \mathrm{~mm}$ radius EDM pre-crack and a focused mesh to accurately capture the strain field at the crack tip. Model 'CCG_CG' has a uniform mesh at the crack tip to allow $0.1 \mathrm{~mm}$ increments of crack growth to be modelled. 
Details of this refined mesh are provided in

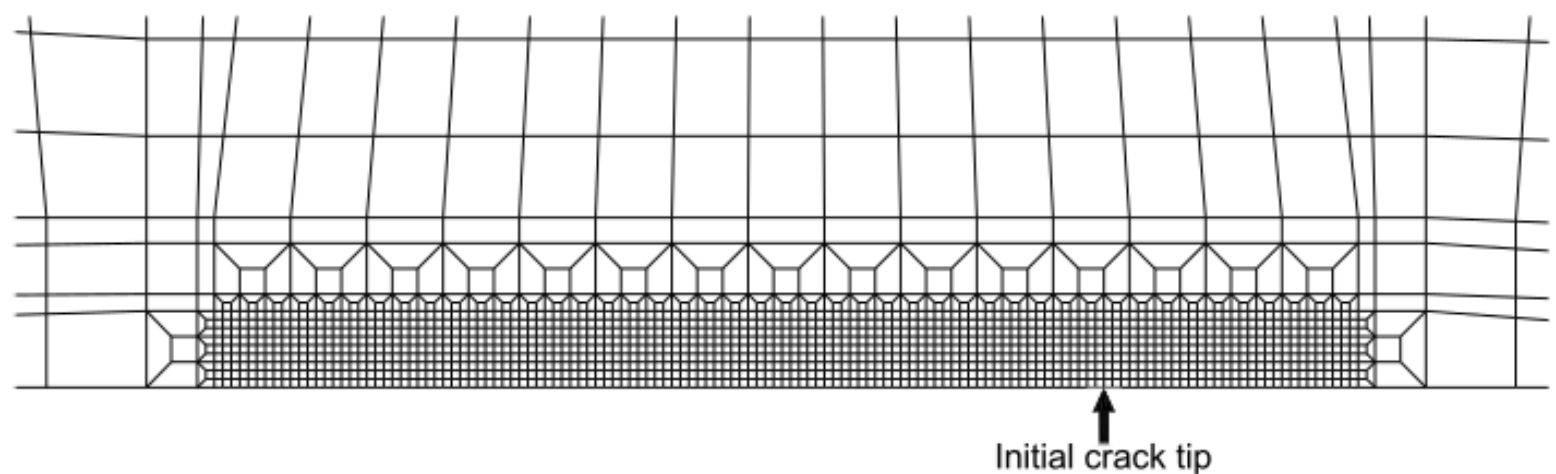

Figure 23. Due to the increased computational expense of the crack growth model, a coarser mesh was applied. This mesh was validated by comparing the results of the two models up to the onset of crack growth. Both models used linear brick elements. Mesh details provided in Table 7.

\begin{tabular}{cccc}
\hline FE Model & $\begin{array}{c}\text { Number of } \\
\text { Elements }\end{array}$ & $\begin{array}{c}\text { Structural Element } \\
\text { Type }\end{array}$ & $\begin{array}{c}\text { Electrical Element } \\
\text { Type }\end{array}$ \\
\hline CCG_CI & 45,300 & C3D8R & DC3D8E \\
CCG_CG & 33,810 & C3D8R & DC3D8E \\
\hline
\end{tabular}

Table 7: Finite element mesh details.

\subsubsection{Boundary Conditions: Model 'CCG_CI'}

Boundary conditions were applied to the planes of symmetry. A concentrated force was applied to a node at the centre of the pin hole. This node was free to move in the $y$ direction (the direction of the applied force) and free to rotate about the $z$ axis to simulate pin rotation in the shackles. All other degrees of freedom with were constrained. The displacement of this node was transmitted to the inside surface of the pin hole via a kinematic coupling. The loading pin was not explicitly modelled to reduce computational expense. It has been shown that this 
modelling simplification has a negligible impact on the PD configuration shown in

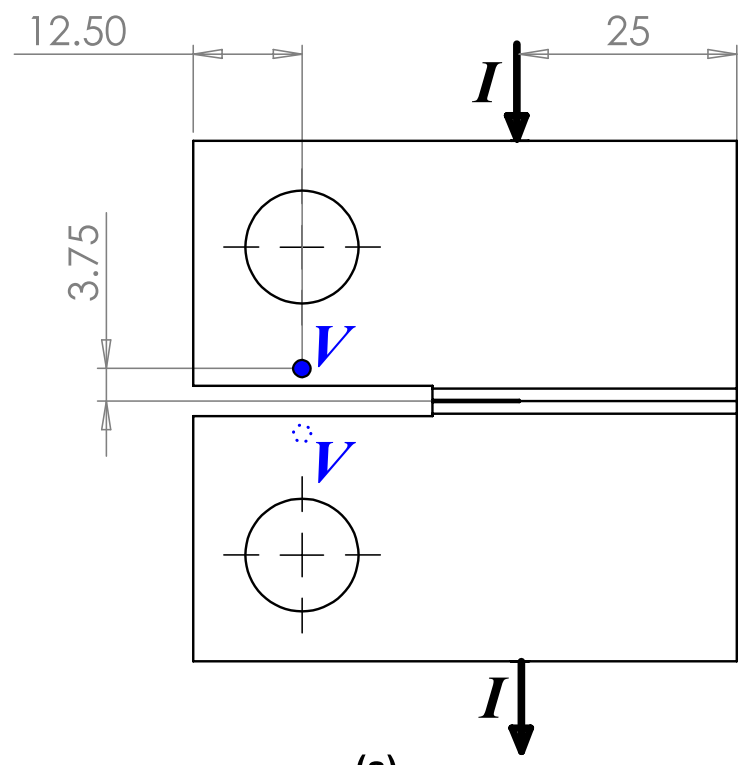

(a)

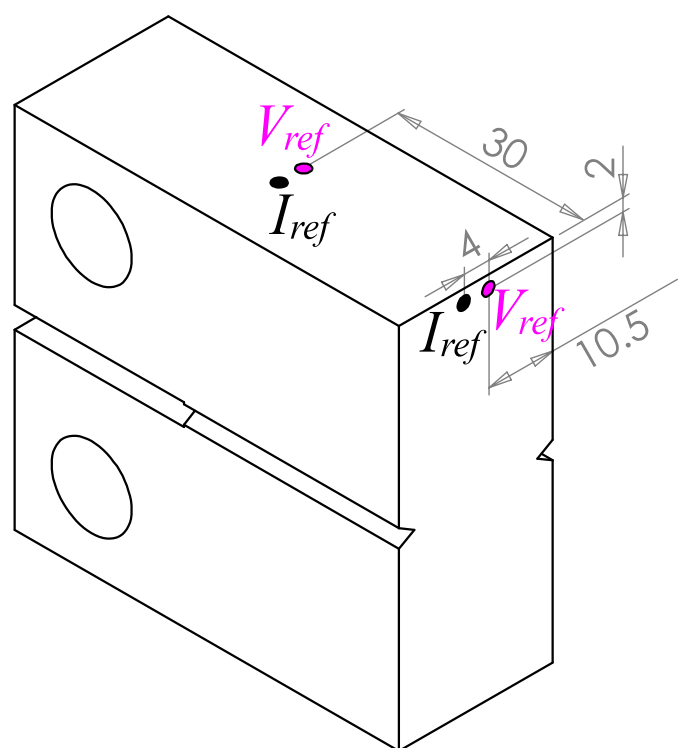

(b)

Figure 4 (Tarnowski et al., 2017). A $12.25 \mathrm{kN}$ force was applied to the FE models. This is equivalent to the $24.5 \mathrm{kN}$ force applied in the experiments due to symmetry. The structural analysis consisted of two load steps: "load-up" and "creep". No creep properties were applied during load-up which assumes that creep strains which occur during the short duration of loadup are negligible.

For the electrical analyses a $0 \mathrm{~V}$ electrical potential was applied to all nodes on the remaining ligament ahead of the crack (the $y$ plane of symmetry). A point current source was applied to a node at the current injection location and the electrical potential was measured at a node at the PD probe location. All electrical results are presented in terms of relative change in resistance so they are independent of the magnitude of the applied current the electrical resistivity applied to the model.

\subsubsection{Boundary Conditions: Model 'CCG_CG'}

The method of applying the load and the initial symmetry boundary conditions were the same as for model 'CCG_CI'. The initial two load steps were also the same, but for every $0.1 \mathrm{~mm}$ increment of crack growth an additional two steps were included. The first of these was to release a line of nodes parallel to the crack front from the symmetry boundary condition applied to the ligament ahead of the crack. The second was to allow creep to occur before the next $0.1 \mathrm{~mm}$ increment in crack length. The rate of crack growth was determined from 

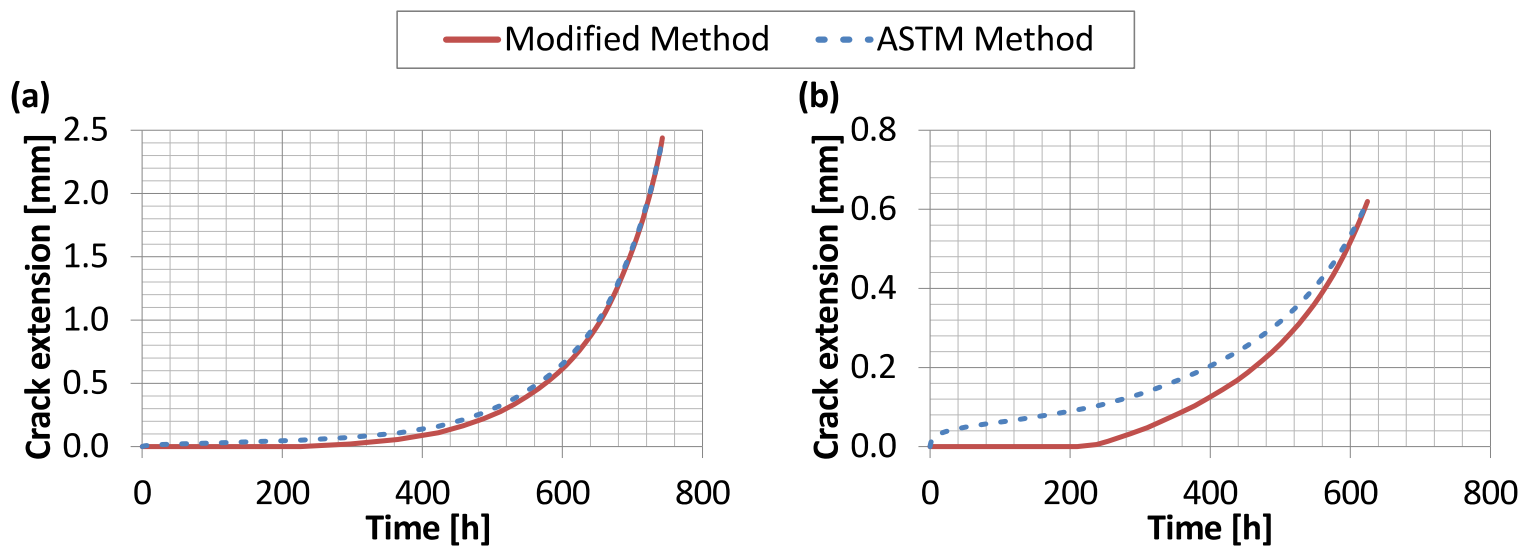

Figure 19(a). The experimental and modelled crack extensions are compared in

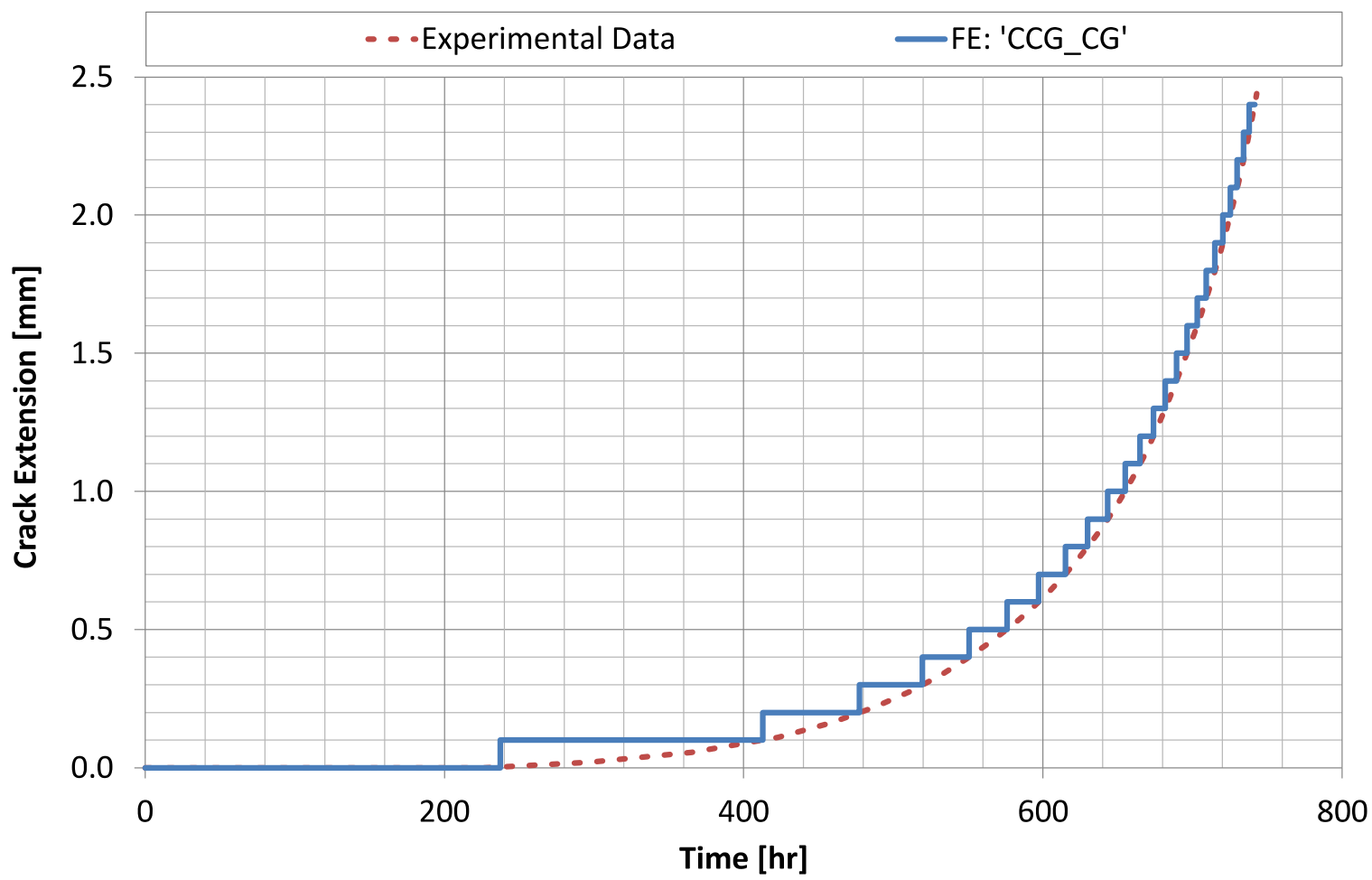

Figure 24 for the duration of the test. This method of simulating crack growth is extremely simplified. It assumes a straight crack front and a continuous, a perfectly sharp crack tip.

For the electrical analyses the boundary conditions were similar to those applied to model 'CCG_CI'. The 0V electrical potential applied to all nodes on the remaining ligament ahead of the crack was updated after each increment of crack growth. 


\subsubsection{Material Properties}

Tensile data for the same cast (55882) of Type $316 \mathrm{H}$ is provided in the edge welded beam benchmark test in R6 (BEGL, 2000). At $550{ }^{\circ} \mathrm{C}$ the elastic modulus, $E$, is $159.75 \mathrm{GPa}$ and a Poisson's ratio, $v$, is 0.294 . The true plastic stress-strain data is provided in Table 8 .

\begin{tabular}{cc}
\hline $\begin{array}{c}\text { True Stress } \\
{[\mathbf{M P a}]}\end{array}$ & $\begin{array}{c}\text { True Strain } \\
{[\mathbf{m m} / \mathbf{m m}]}\end{array}$ \\
\hline 116.2 & 0.000 \\
191.9 & 0.002 \\
228.7 & 0.010 \\
257.2 & 0.020 \\
332.9 & 0.050 \\
425.7 & 0.100 \\
530.8 & 0.200 \\
606.1 & 0.300 \\
\hline
\end{tabular}

Table 8: True plastic stress-strain data for Type $316 \mathrm{H}$ stainless steel (cast 55882).

A primary and secondary creep law is provided in Appendix 16 of RCC-MR (RCC-MR, 2002) and is reproduced here in Equation (4):

$$
\varepsilon^{c}=\left\{\begin{aligned}
C_{1} t^{C_{2}} \sigma^{n_{1}}, & t \leq t_{f p} \\
C_{1} t_{f p}{ }^{C_{2}} \sigma^{n_{1}}+C \sigma^{n}\left(t-t_{f p}\right), & t>t_{f p}
\end{aligned}\right.
$$

In this creep law $\varepsilon^{c}$ is the total creep strain (in $\mathrm{mm} / \mathrm{mm}$ ), $C_{1}, C_{2}, C, n_{1}$ and $n$ are material constants and $t$ is time (in hours). The transition from primary to secondary creep, occurs at time $t_{f p}$. This corresponds to the time at which the primary creep rate is equal to the secondary creep rate and can be calculated from Equation (5):

$$
t_{f p}=C_{3} \sigma^{n_{3}}
$$

where:

$$
\begin{gathered}
C_{3}=\left(\frac{C}{C_{1} C_{2}}\right)^{\frac{1}{C_{2}-1}} \\
n_{3}=\frac{n-n_{1}}{C_{2}-1}
\end{gathered}
$$


At times less than $t_{f p}$ the total creep strain is due to primary creep only. After this the total creep strain is equal to the primary creep strain at the transition time plus a secondary creep component.

Rather than using the material constants provided in RCC-MR, which were originally derived from tests performed on Type $316 \mathrm{LN}$, new constants have been derived based on 11 tests performed on Type 316H (Bettinson, 2001; Mehmanparast, 2012). These tests were all performed at $550{ }^{\circ} \mathrm{C}$ and at stresses between $257 \mathrm{MPa}$ and $366 \mathrm{MPa}$. The derived constants are provided Table 9.

\begin{tabular}{cc}
\hline Parameter & Value \\
\hline$C_{1}$ & $2.42 \times 10^{-17}$ \\
$C_{2}$ & 0.53 \\
$n_{1}$ & 5.37 \\
$C$ & $9.05 \times 10^{-36}$ \\
$n$ & 12.20 \\
\hline
\end{tabular}

Table 9: Average creep law coefficients for $316 \mathrm{H}$ at $550^{\circ} \mathrm{C}$. Stress in MPa, time in $h$.

This creep law was applied to the FE model via a user subroutine which assumed strain hardening conditions. A sensitivity study was used to demonstrate that the difference between strain hardening and time hardening was negligible.

\subsection{Finite Element Validation: Results}




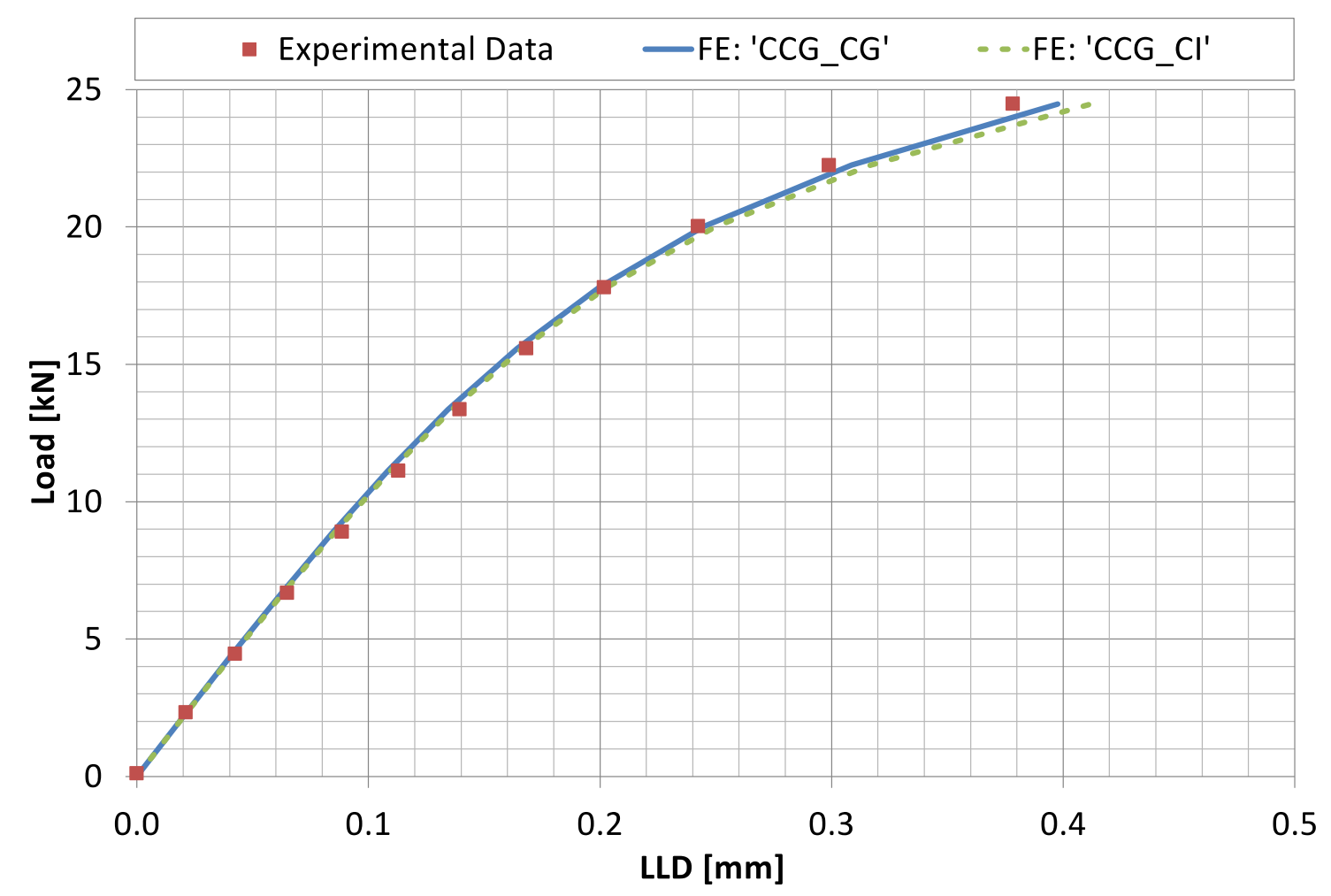

Figure 25 compares the structural response of the FE models with the experimental data for specimen CCG316_CT01 during load-up. The FE predictions are in good agreement with the experimental data which provides confidence in the tensile material properties and structural modelling assumptions. The response of the two models is almost identical which demonstrates that the differences between them (crack tip acuity and mesh density) do not significantly influence the global response of the specimen during load-up.
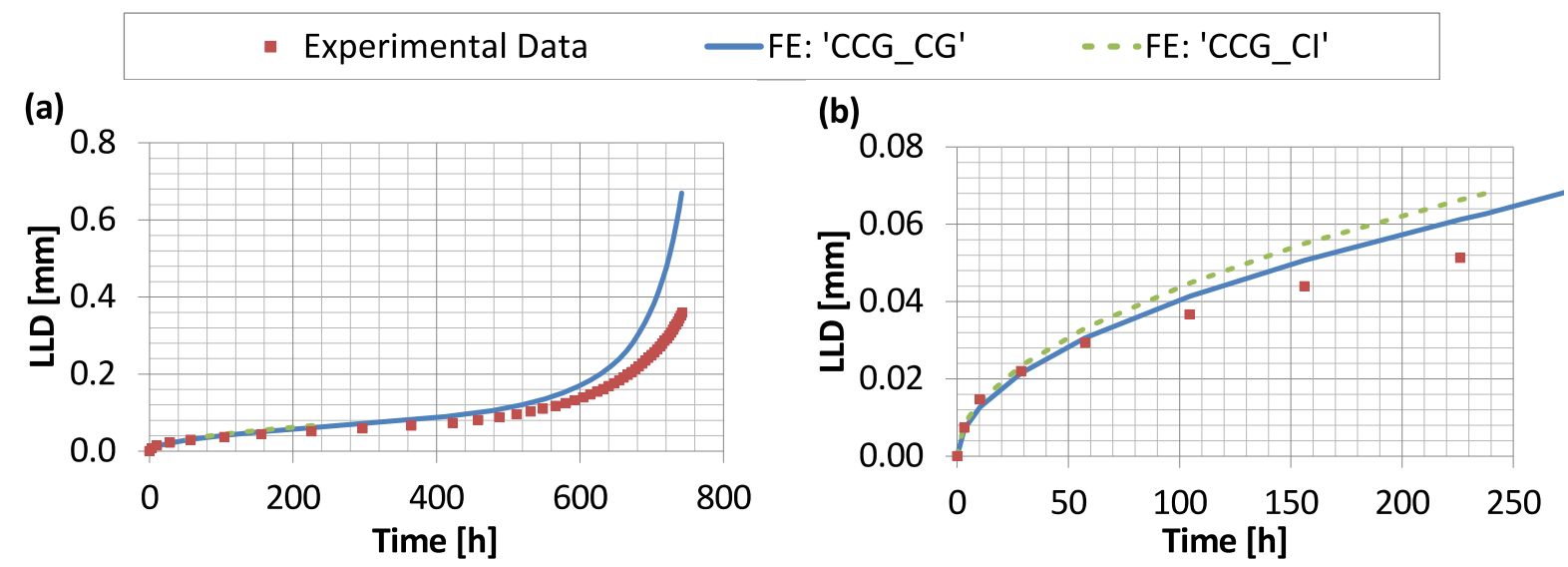

Figure 26 compares the time dependent response of the FE models with the experimental data. 

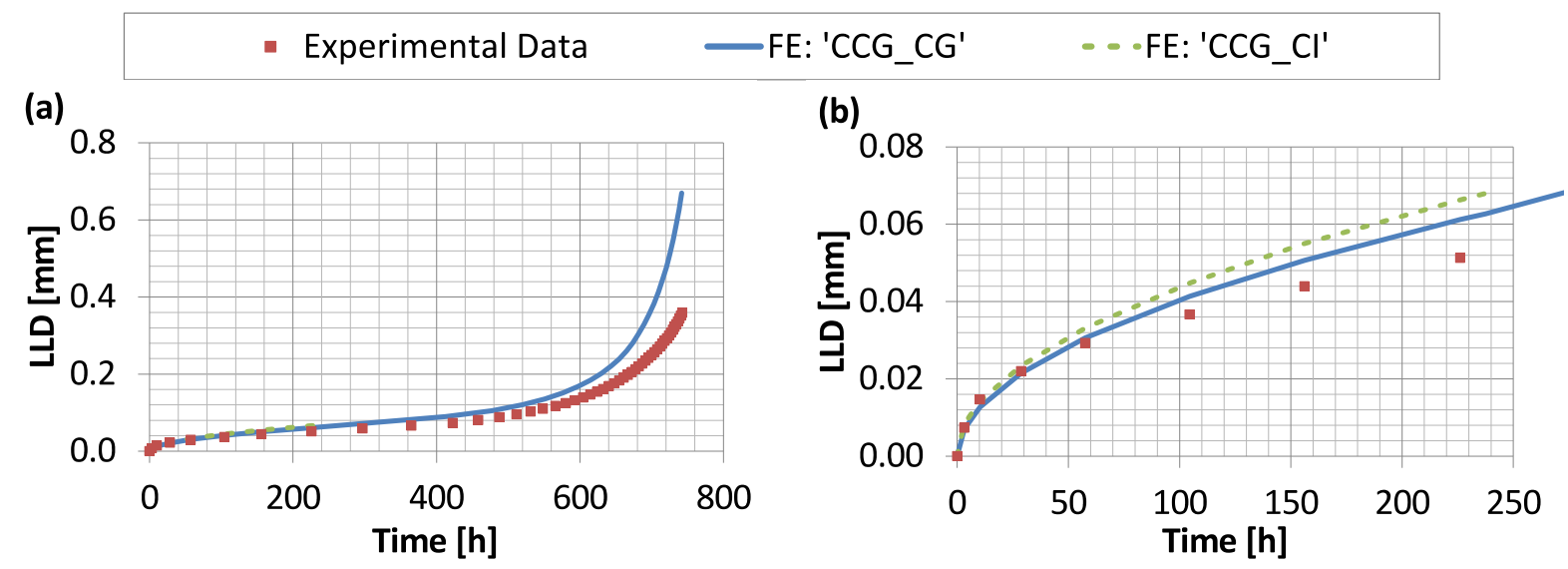

Figure 26(a) shows data for the entire test, whilst
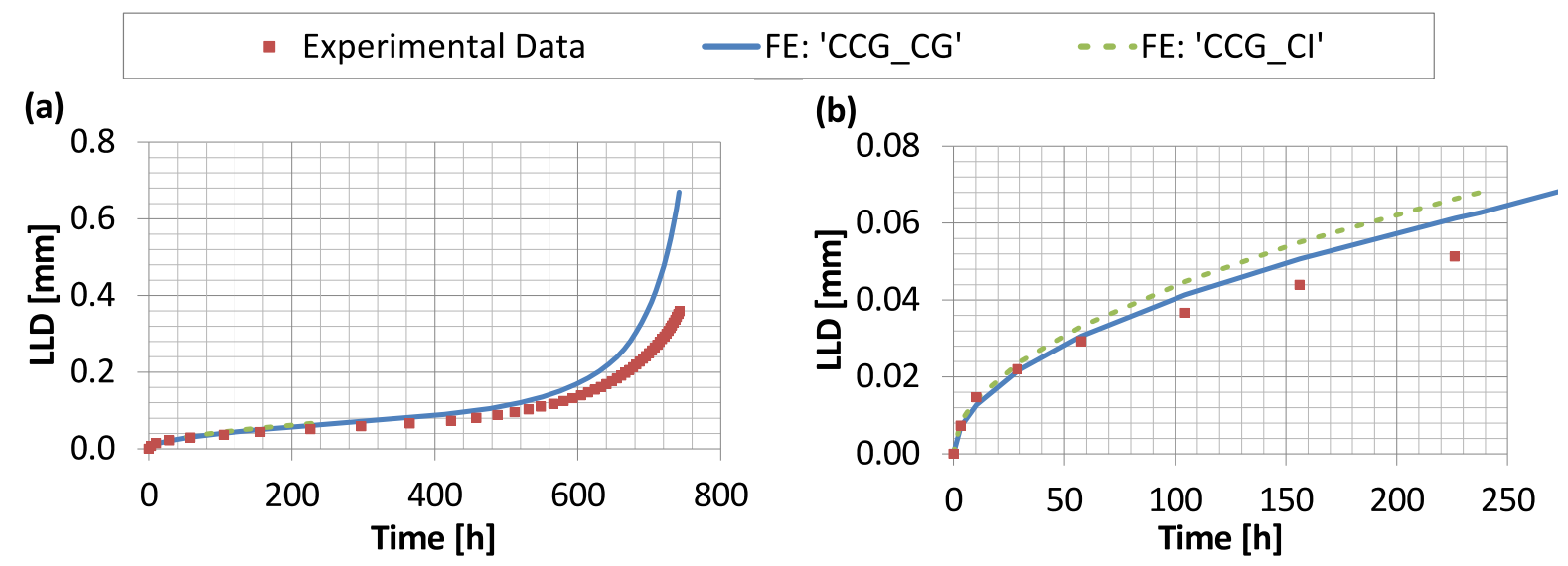

Figure 26(b) only shows the data for the initial 250 hours. The FE models are in good agreement with the experimental data up to the onset of crack growth ( 234 hours) which provides confidence in the creep properties applied to the model. The response of the two models is also very similar which demonstrates that the differences between them (crack tip acuity and mesh density) do not significantly influence the global response of the specimen during creep. After crack initiation, the LLD is overestimated by model ' $\mathrm{CCG} C \mathrm{CG}$ ' compared to the experimental data. This is most significant towards the end of the test where the LLD predicted by the FE model is almost double the experimental value. The most likely source of this discrepancy is the simplified crack morphology in the FE model. Uneven crack extension and discontinuous cracking will both reduce the LLD compared to the continuous, straight fronted crack which is modelled and both of these phenomena have been observed in specimen CCG316_CT01 (see 


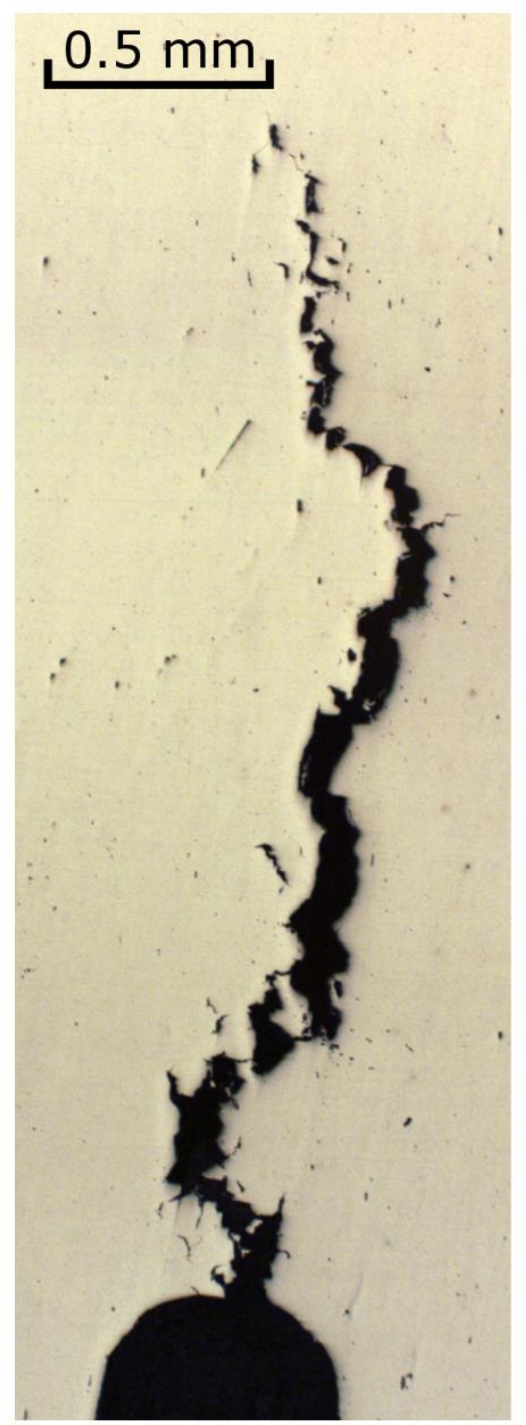

(a)

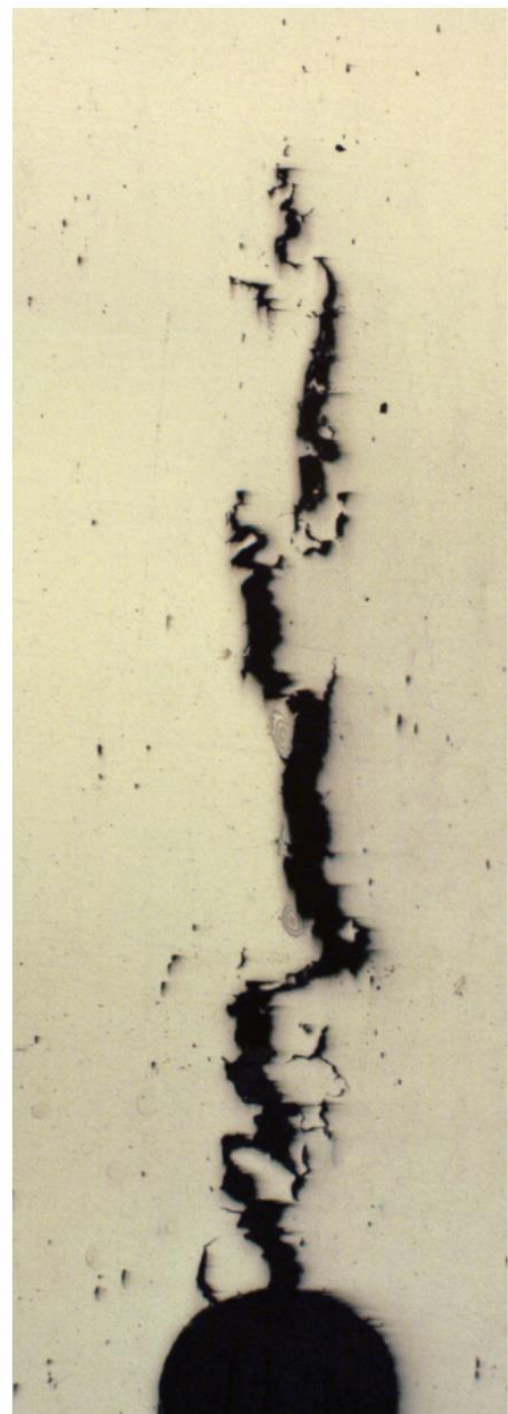

(b)

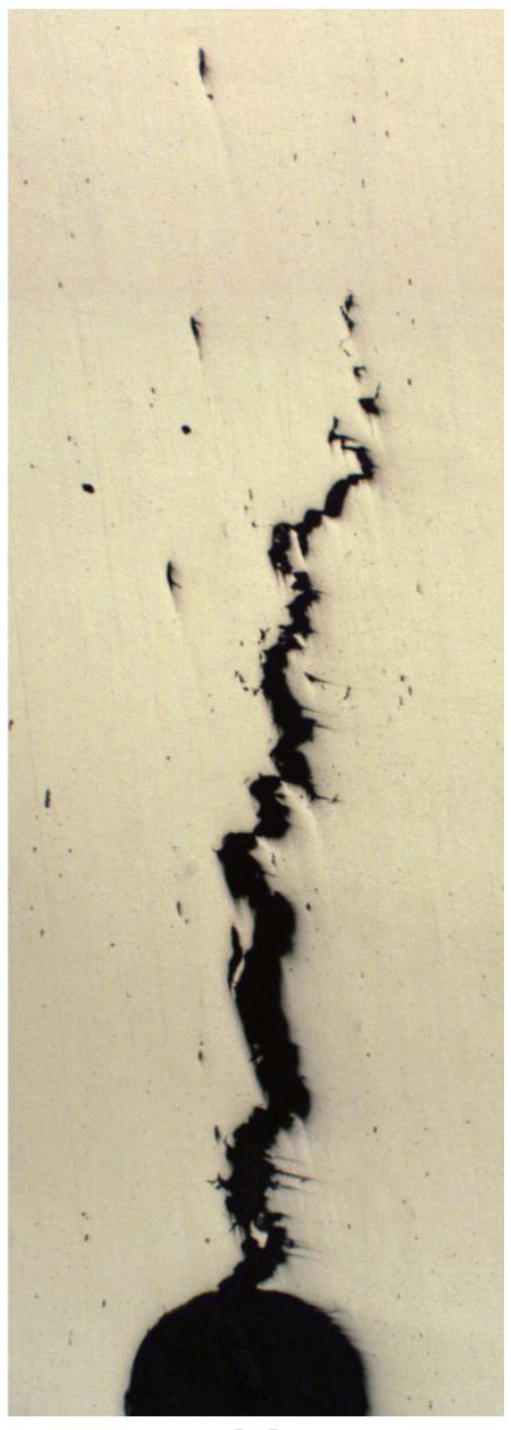

(c)

Figure 12 and 


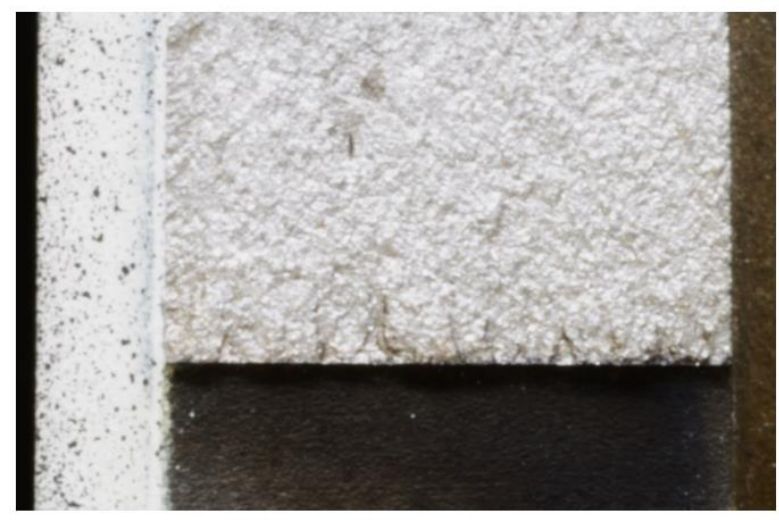

(a)

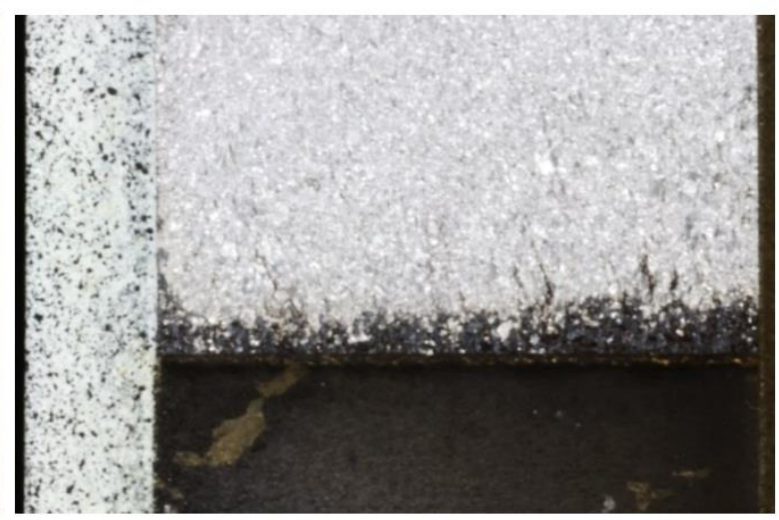

(b)

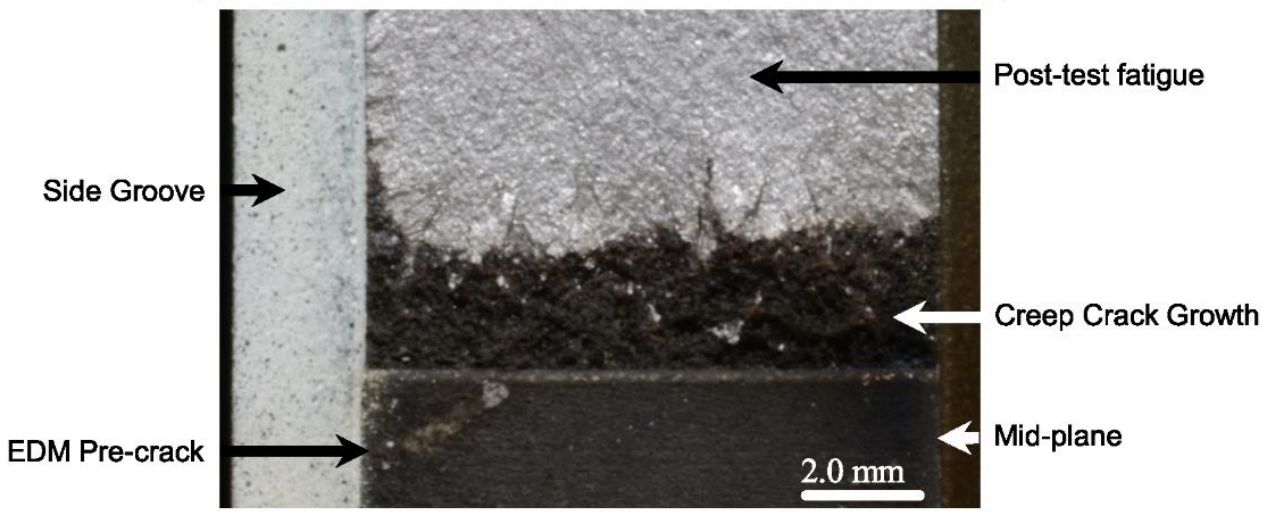

(c)

Figure 18).
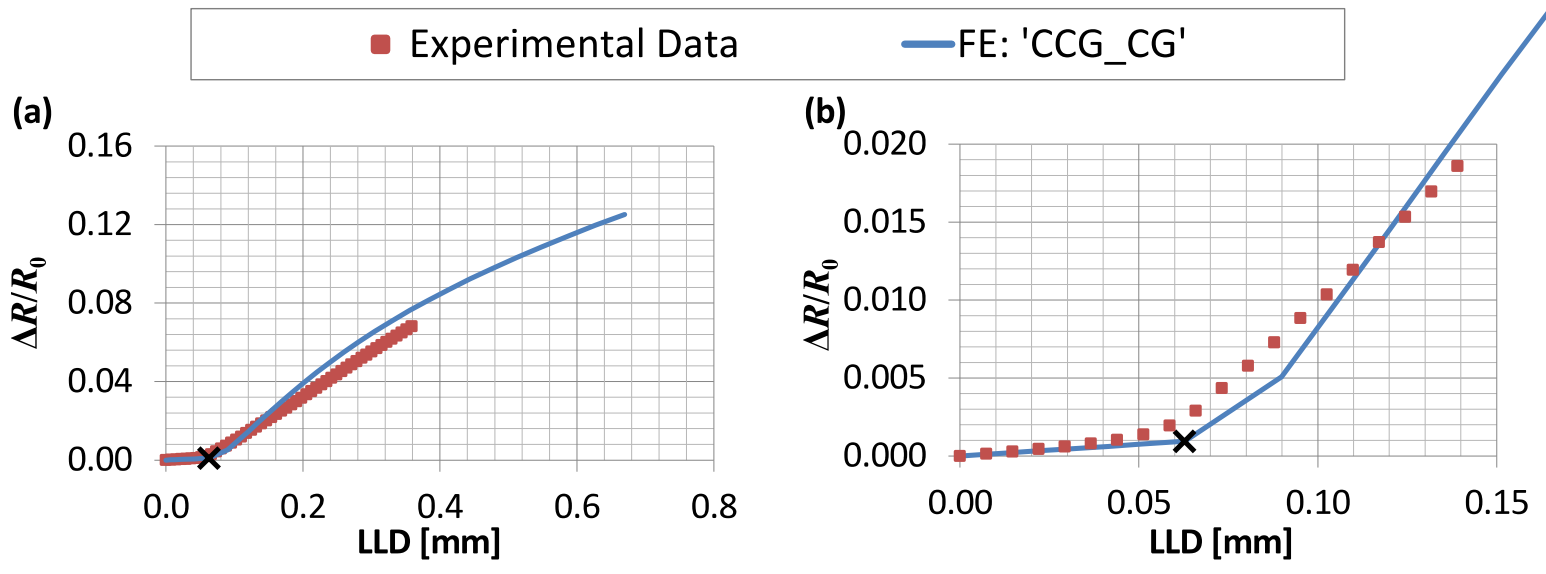

Figure 27 shows the relationship between relative change in resistance and LLD obtained experimentally and predicted by the FE model which includes crack growth. 

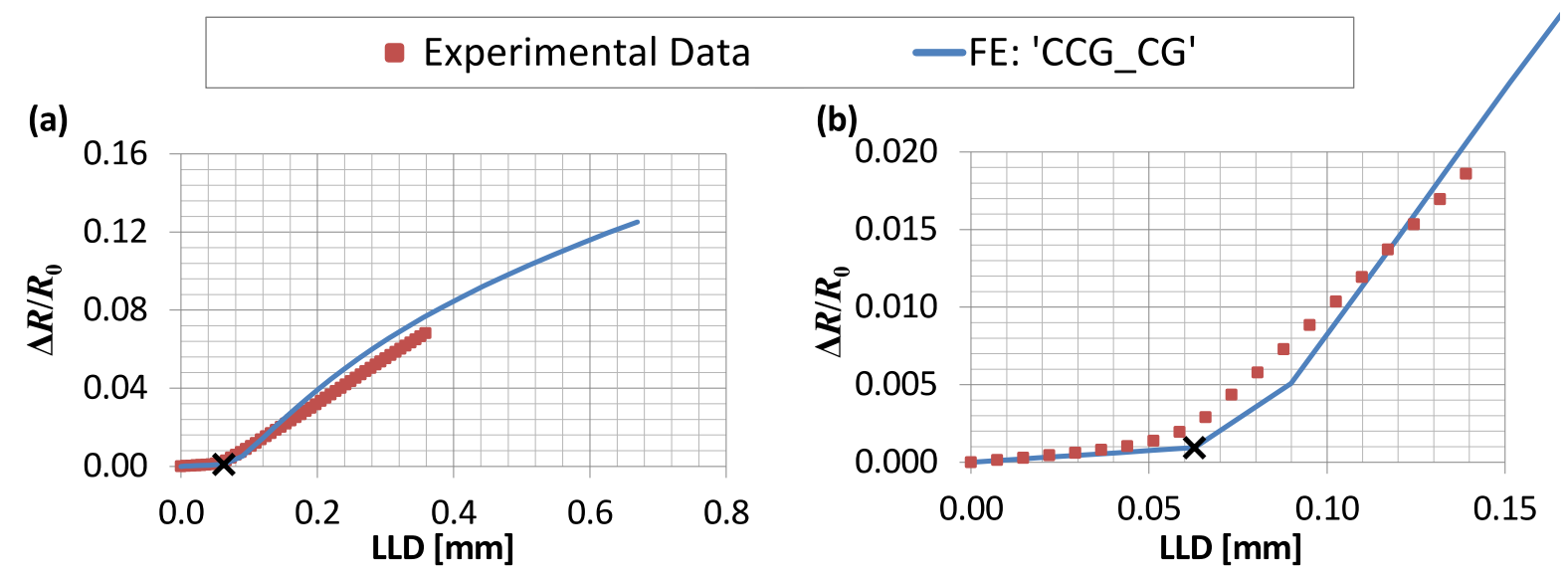

Figure 27(a) shows data for the entire test, whilst
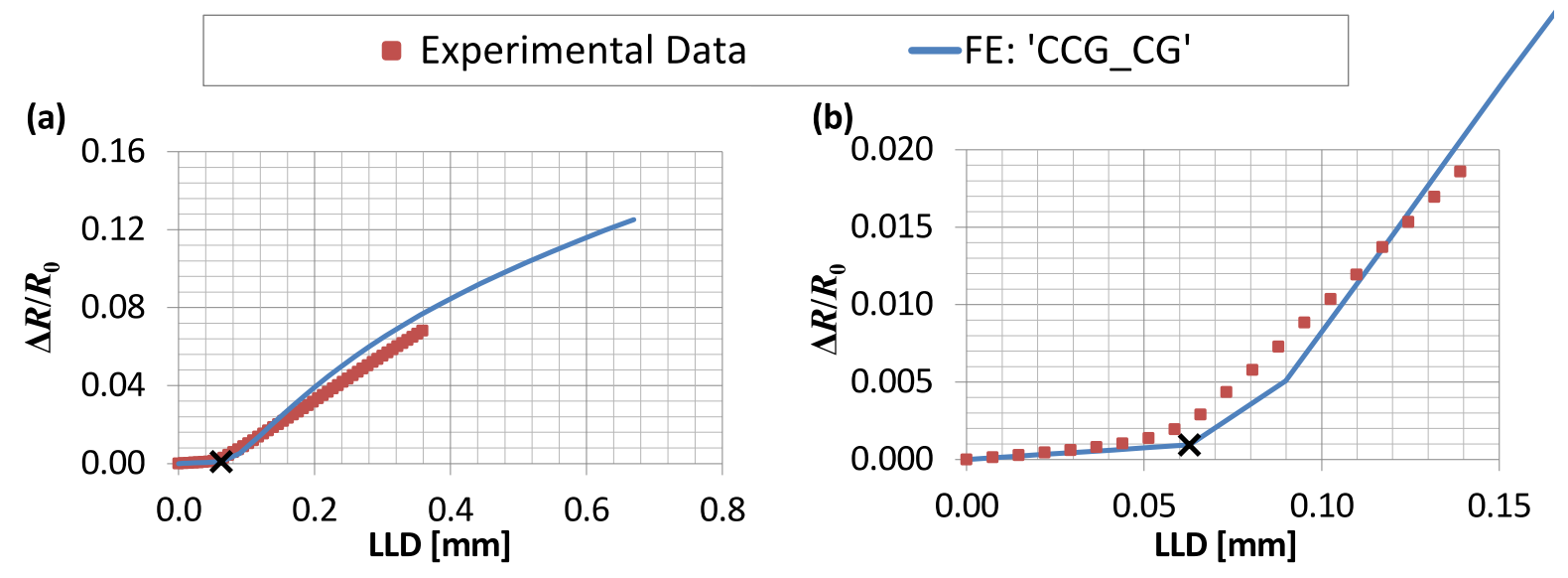

Figure 27(b) focuses on the initial part of the test. The onset of crack growth in the FE model is identified by ' $\mathrm{X}$ '.

Prior to any crack growth, the relationship predicted by the FE model is in good agreement with the experimental observations. At the onset of crack growth the FE model predicts an increase in gradient similar to that observed experimentally which confirms that this feature on a plot of resistance (or PD) vs. LLD corresponds to the onset of crack growth. The increase in gradient in the FE model occurs in two stages. The initial increase in gradient, at a LLD of $\sim 0.06 \mathrm{~mm}$, corresponds to the first $0.1 \mathrm{~mm}$ increment of crack extension. The second increase in gradient, at a LLD of $\sim 0.09 \mathrm{~mm}$, corresponds to the next $0.1 \mathrm{~mm}$ increment of crack extension. This confirms that strain still influences the PD response during the early stages of crack growth and explains why the increase in gradient observed experimentally is not abrupt but occurs over a few data points.

After the onset of crack growth, the FE over-predicts both the relative change in resistance and the LLD. The differences in LLD are due to the simplified crack morphology in the FE model 
as discussed previously. The differences in resistance are likely to be for the same reason. Discontinuous cracking and asperities on the fracture surfaces observed experimentally will allow the current to short across the crack faces, reducing the measured resistance of the specimen compared to the FE model.

During crack growth, the FE model predicts a gradually decreasing gradient compared to the approximately linear response observed in the interrupted tests however, a similar reducing gradient was observed in the test which was continued to rupture (see

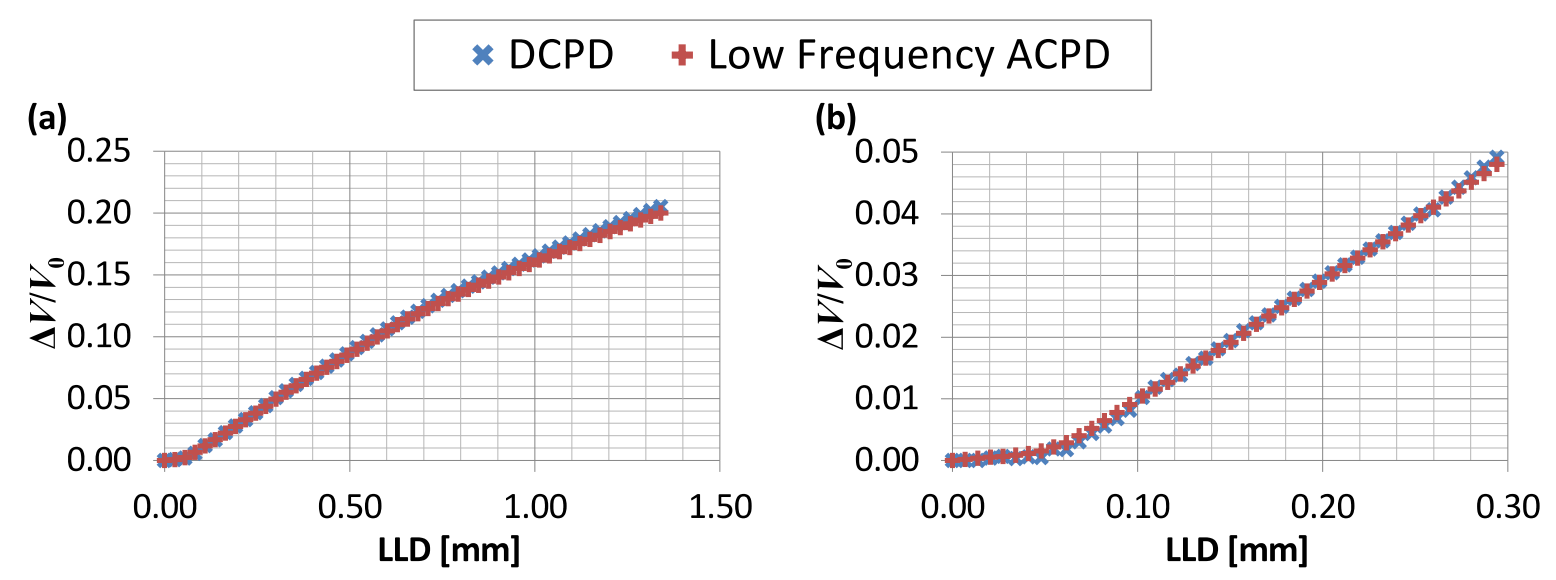

Figure 8(a)). This suggests that the relationship between resistance (or PD) and LLD is nonlinear for large crack extensions so, if using the intersection of two linear regression fits to identify the value of $R_{0}$ (or $V_{0}$ ), these should only be applied to a portion of the data either side of the increase in gradient and not all of the data.

As well as confirming that the change in gradient corresponds to crack initiation, model 'CCG_CG' can also be used to estimate the significance of strain on the subsequent PD measurements. 


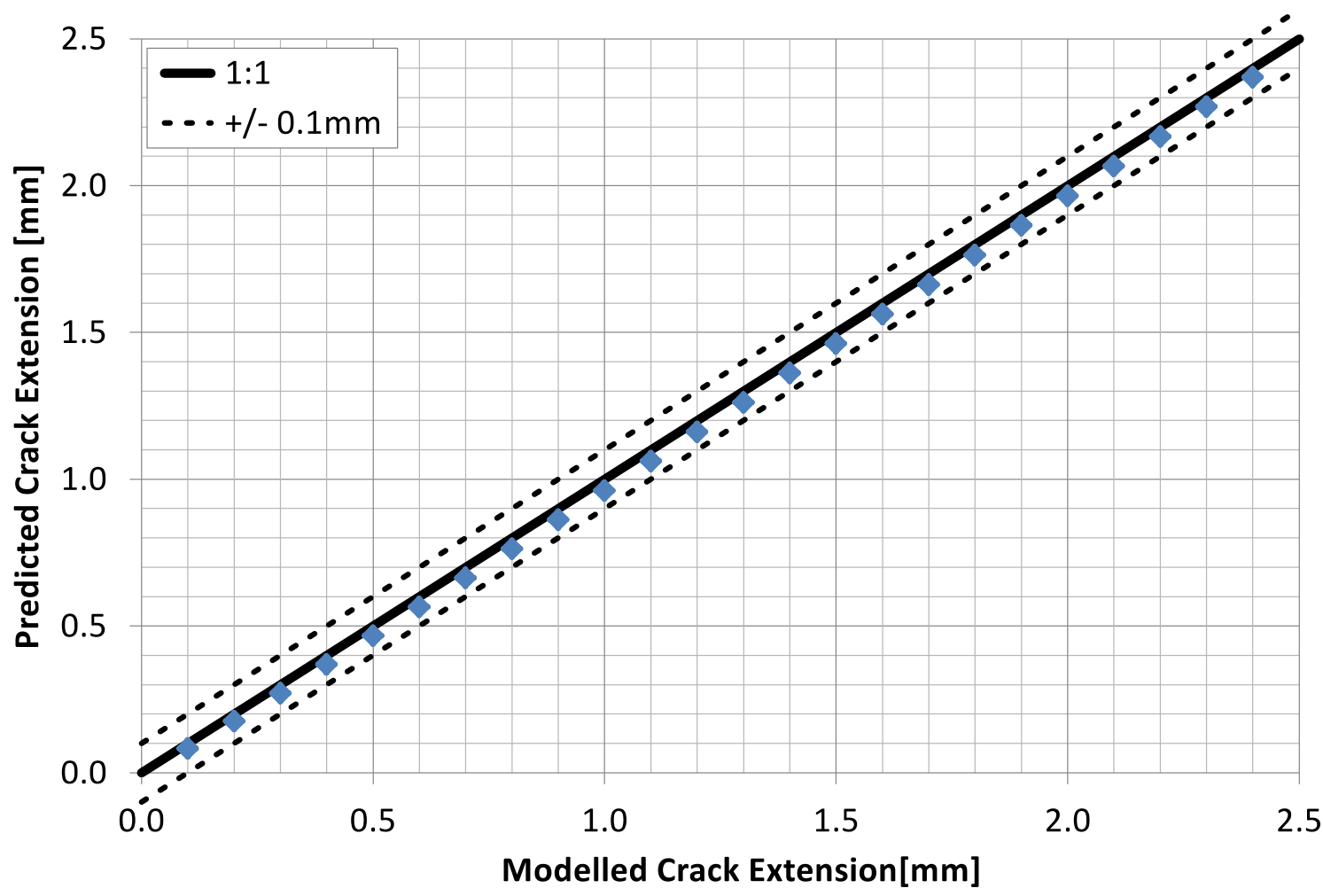

Figure 28 compares the crack extension predicted from the PD response (obtained from the FE model) with the modelled crack extension. It demonstrates excellent agreement $( \pm 0.1 \mathrm{~mm})$ for all crack lengths confirming that, for this material, the influence of strain on the PD measurements is small compared to the influence of crack extension.

\section{Other Materials}

To confirm that a similar increase in gradient on a plot of PD (or resistance) vs. LLD is observed for a range of materials, temperatures and test durations, experimental data obtained from CCG tests performed by EDF Energy on austentic Type 316H (Dean and Gladwin, 2004), austentic Esshete 1250 (Gladwin, 1998), and ferritic $1 / 2 \mathrm{Cr}^{1} / 2 \mathrm{Mo}^{1 / 4} \mathrm{~V}$ (Baker and Gladwin, 2004) has been re-analysed. Unlike the interrupted CCG tests described above, these tests were performed on specimens that contained a fatigue pre-crack rather than an EDM pre-crack and the PD measurements were obtained using a DCPD system. A reference PD measurement was not implemented so the PD data is susceptible to fluctuations due to small changes in temperature.

For each of these tests an increase in gradient was identified on a plot of PD against LLD similar to those observed during the interrupted CCG tests described above. Typical examples are provided in 
(a)

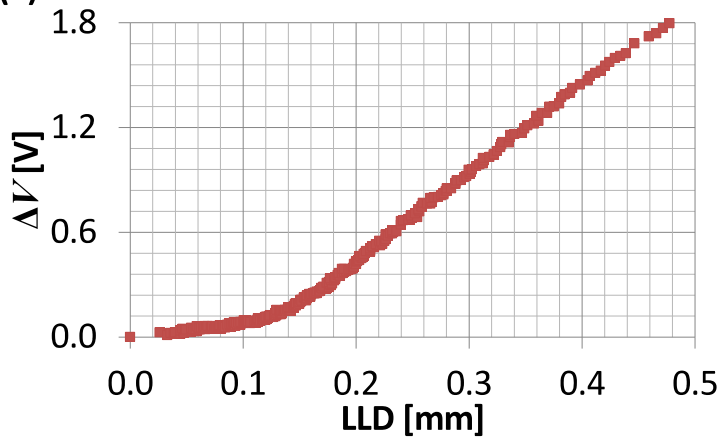

(b)

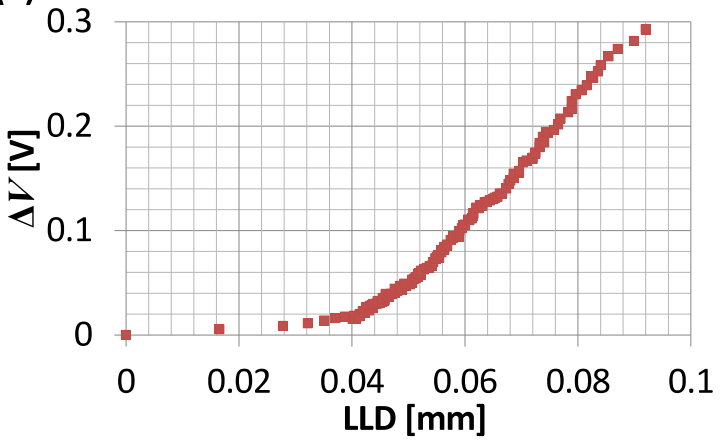

Figure 29. The consistent occurrence of this phenomenon suggests that the proposed method of identifying creep crack initiation can be applied to a wide range of materials, temperatures and test durations.

For some of the tests the increase in gradient was less obvious than others due to fluctuations in the PD data. These were probably due to changes in temperature which could have been supressed by implementing a reference PD measurement. The reduced noise and thermal stability of the low frequency ACPD system described above may also have improved the data. This highlights the need for careful consideration of the experimental setup in order to accurately analyse CCG test data.

It has been observed that the proposed modified method of interpreting the PD data has a significant influence on the measured incubation period. To investigate this further, the incubation period corresponding $0.2 \mathrm{~mm}$ of crack extension was identified each test using both methods: Once using the method in ASTM E1457, $t_{0.2}^{A S T M}$, and once using the proposed modified method, $t_{0.2}^{M O D}$. The results are presented separately for each material in the following sub-sections.

\section{$5.1 \quad$ Type $316 \mathrm{H}$}

The thirteen CCG tests performed on austenitic Type 316H stainless steel are summarised in Table 10. For each test, this table includes the plane strain reference stress, $\sigma_{\text {ref }}$, the test duration, $t_{f}$, the incubation periods corresponding to the two methods of interpreting the PD data, and the ratio of these two incubation periods. All of these tests were performed at a temperature of $550{ }^{\circ} \mathrm{C}$.

\begin{tabular}{|c|c|c|c|c|c|}
\hline Test ID & $\begin{array}{c}\sigma_{r e f} \\
{[\mathrm{MPa}]}\end{array}$ & $\begin{array}{c}t_{f} \\
{[\mathbf{h}]}\end{array}$ & $\begin{array}{c}t_{0.2}^{A S T M} \\
{[\mathbf{h}]}\end{array}$ & $\begin{array}{l}t_{0.2}^{M O D} \\
{[\mathbf{h}]}\end{array}$ & $\frac{t_{0.2}^{M O D}}{t_{0.2}^{A S T M}}$ \\
\hline 2D2/2 CT1 & 132 & 16630 & 1569 & 3252 & 2.1 \\
\hline
\end{tabular}




\begin{tabular}{cccccc}
\hline 2D2/2 CT2 & 152 & 4698 & 970 & 1788 & 1.8 \\
2D2/2 CT3 & 251 & 170 & 62 & 64 & 1.0 \\
2D2/2 CT4 & 196 & 1921 & 508 & 864 & 1.7 \\
2D2/2 CT5 & 200 & 1589 & 394 & 631 & 1.6 \\
2D2/2 CT20 & 128 & 17850 & 983 & 1500 & 1.5 \\
1C2/3 CT5 & 234 & 146 & 29 & 35 & 1.2 \\
1C2/3 CT6 & 197 & 1081 & 161 & 258 & 1.6 \\
1C2/3 CT11 & 258 & 287 & 20 & 26 & 1.3 \\
2B1/2 CT14 & 244 & 140 & 24 & 31 & 1.3 \\
2B1/2 CT15 & 252 & 62 & 6 & 12 & 2.0 \\
2B1/2 CT16 & 160 & 3572 & 356 & 1602 & 4.5 \\
2B1/2 CT17 & 169 & 1537 & 199 & 595 & 3.0 \\
\hline
\end{tabular}

Table 10: Summary of re-analysed CCG tests performed on Type 316H.

The ratio of the time for $0.2 \mathrm{~mm}$ of crack extension to occur based on the modified method of interpreting the PD data and the ASTM method varies from 1.0 to 4.5. The average value for all 13 tests is 1.9. A ratio of 1.0 identifies a test where the influence of strain on PD, prior to the onset of crack growth, is negligible so the two methods of interpreting the PD data yield almost identical incubation periods. For tests with higher ratios, the influence of strain on the PD measurement is much greater. The test with the highest ratio is $2 \mathrm{~B} 1 / 2 \mathrm{CT} 16$. For this test, the increase in gradient, which is believed to correspond to the onset of crack growth, occurs after 1100 hours. At this time, if the PD is interpreted in accordance with ASTM E1457, $\sim 0.37 \mathrm{~mm}$ of crack extension is predicted however, the results of this study suggest that this is entirely due to the accumulation of creep strain.

\subsection{Esshete 1250}

The six CCG tests performed on austenitic Esshete 1250 are summarised in Table 11. These tests were performed at temperatures ranging from $550{ }^{\circ} \mathrm{C}$ to $600{ }^{\circ} \mathrm{C}$. The temperature of each test is included in the table.

\begin{tabular}{ccccccc}
\hline Test ID & $\begin{array}{c}\text { Temp. } \\
{\left[{ }^{\circ} \mathbf{C}\right]}\end{array}$ & $\begin{array}{c}\boldsymbol{\sigma}_{\text {ref }} \\
{[\mathbf{M P a}]}\end{array}$ & $\begin{array}{c}t_{f} \\
{[\mathbf{h}]}\end{array}$ & $\begin{array}{c}t_{0.2}^{A S T M} \\
{[\mathbf{h}]}\end{array}$ & $\begin{array}{c}t_{0.2}^{\text {MOD }} \\
{[\mathbf{h}]}\end{array}$ & $\frac{t_{0.2}^{\text {MOD }}}{t_{0.2}^{A S T M}}$ \\
\hline 1 & 550 & 253 & 530 & 12 & 267 & 22.6 \\
2 & 570 & 239 & 664 & 485 & 496 & 1.0 \\
3 & 600 & 208 & 168 & 93 & 106 & 1.1 \\
4 & 570 & 219 & 794 & 112 & 499 & 4.5
\end{tabular}




\begin{tabular}{ccccccc}
\hline 5 & 550 & 225 & 1698 & 1547 & 1603 & 1.0 \\
6 & 570 & 198 & 1146 & 566 & 835 & 1.5 \\
\hline
\end{tabular}

Table 11: Summary of re-analysed CCG tests performed on Esshete 1250.

The ratio $t_{0.2}^{M O D} / t_{0.2}^{A S T M}$ varies from 1.0 to 22.6 for these six tests considered here. The highest ratio corresponds to test ID 1 and the results for this test, interpreted using both methods, are compared in
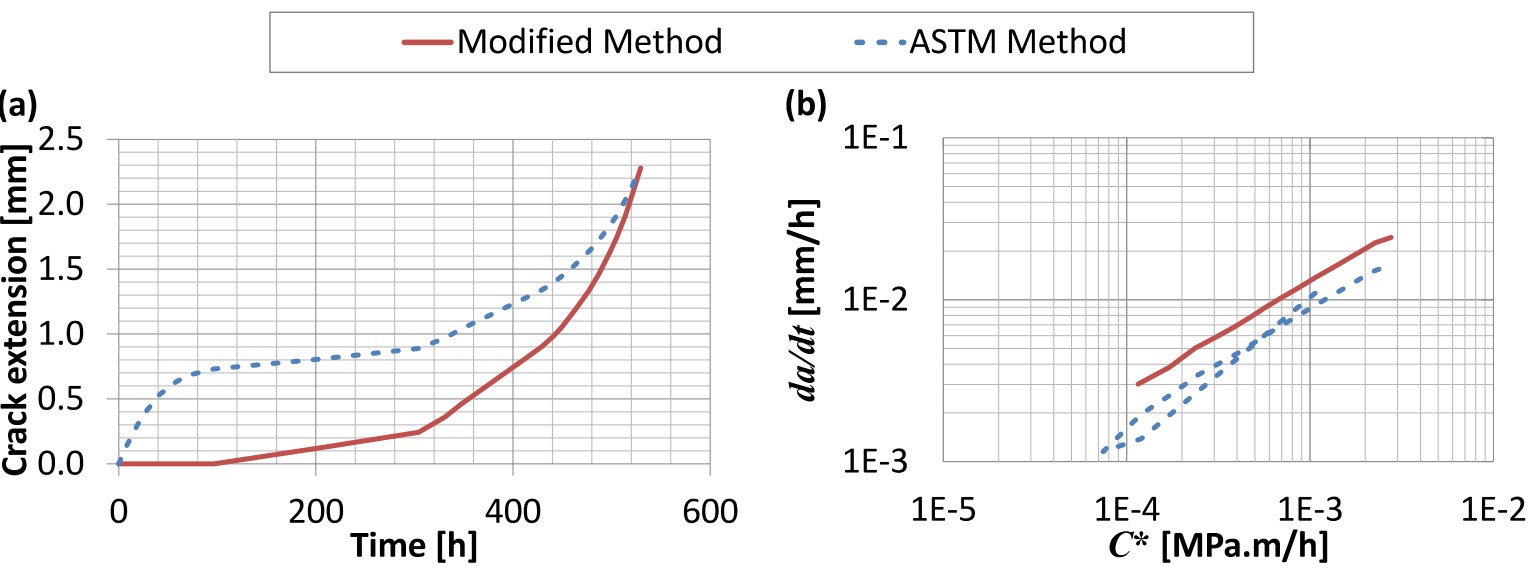

Figure 30.

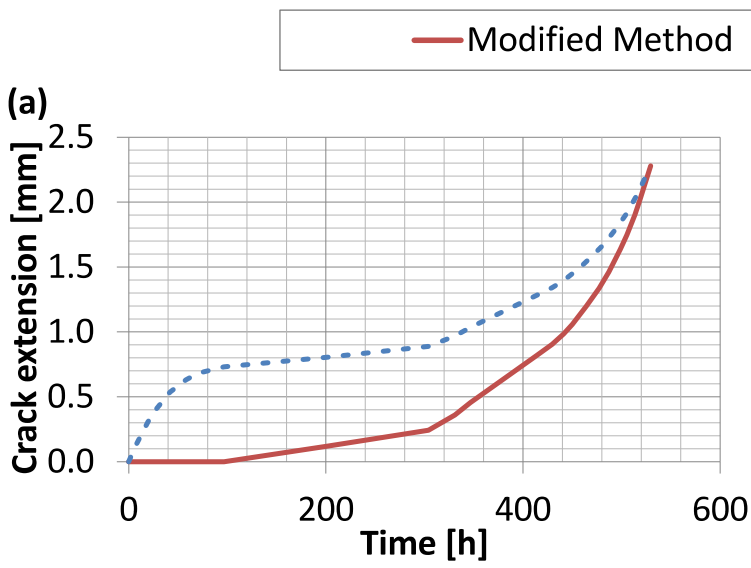

\section{-..ASTM Method}

(b)

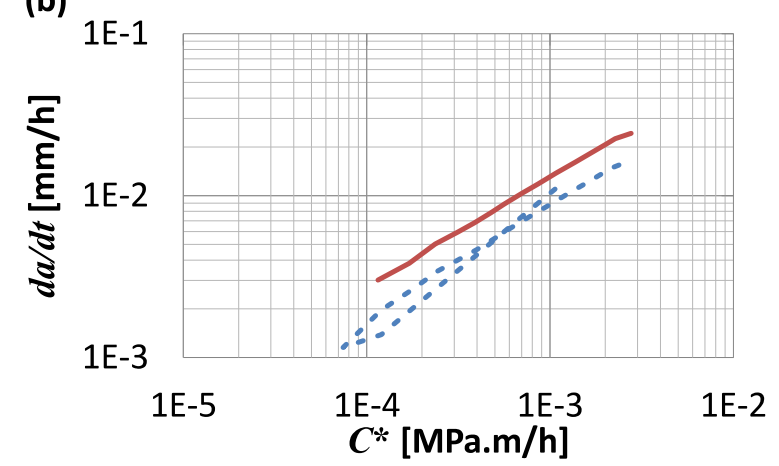

Figure 30(a) shows the variation in crack extension with time and 

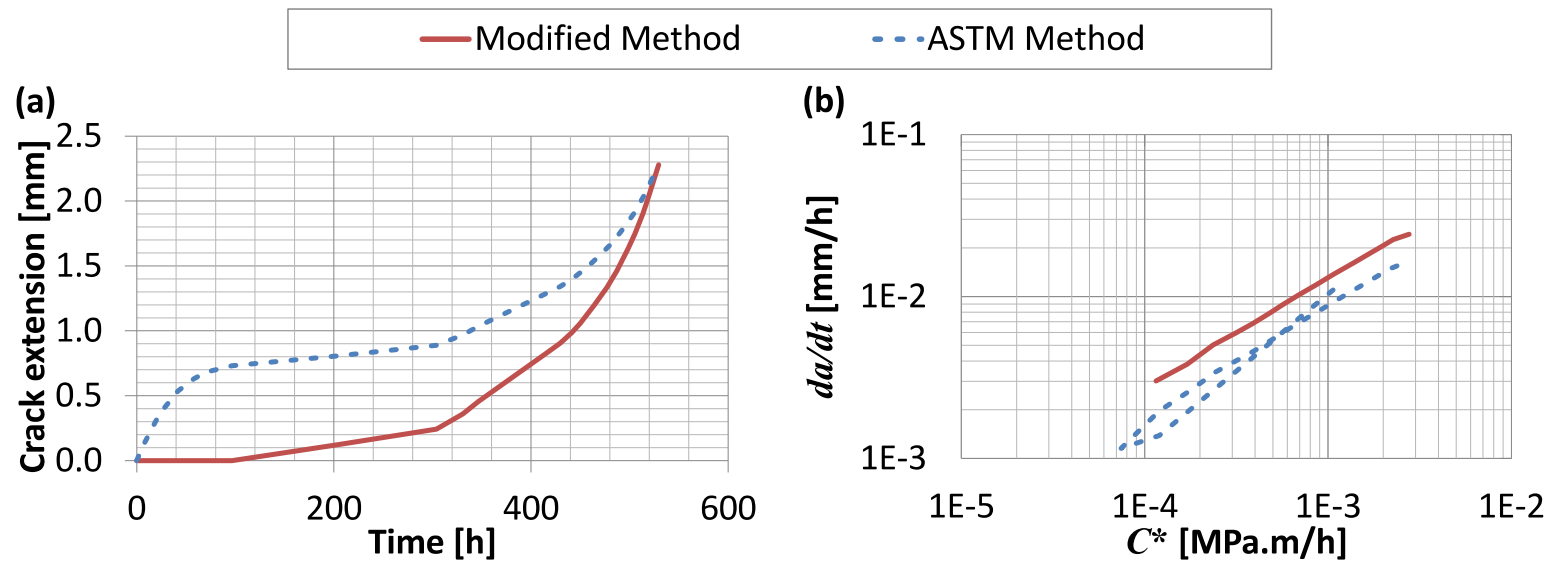

Figure 30(b) shows the correlation between the crack growth rate and $C^{*}$ for all data that meets the validity limits specified in ASTM E1457.

Similar to the interrupted tests discussed previously, the ASTM method predicts immediate crack growth, whilst the modified method identifies an incubation period. The duration of this incubation period is 119 hours and after this time, the ASTM method predicts $\sim 0.75 \mathrm{~mm}$ of spurious crack extension. This is much greater than the $0.2 \mathrm{~mm}$ engineering definition of crack initiation and is the cause of the large $t_{0.2}^{M O D} / t_{0.2}^{A S T M}$ ratio. The spurious crack extension is also comparable to the actual crack growth that occurs during the test $(2.3 \mathrm{~mm})$ and therefore influences the calculated crack growth rates. This is demonstrated in
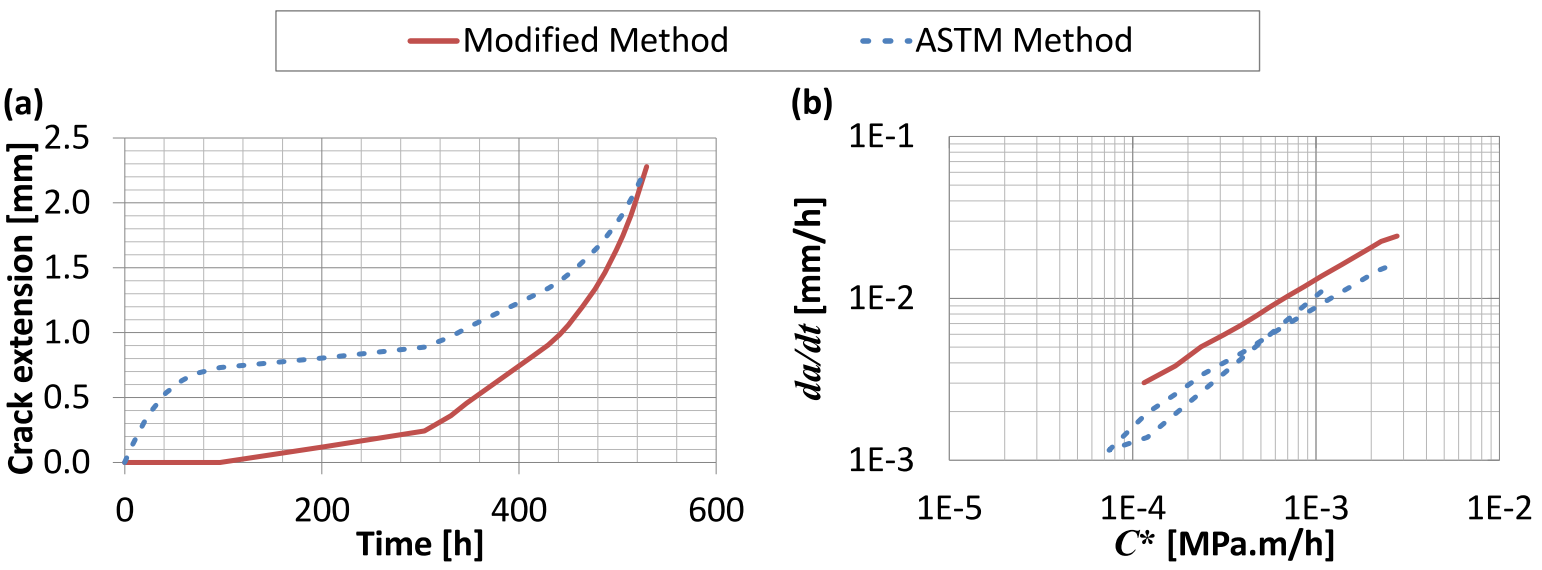

Figure 30(b) where the modified method predicts a steady-state crack growth rate which is $\sim 1.5$ times faster than the ASTM method. The modified method also removes the tail from this plot, similar to the interrupted tests, which again demonstrates that this is caused by erroneously interpreting strains as crack growth.

\section{$5.3 \quad \underline{1 / 2 \mathrm{Cr}^{1} / 2 \mathrm{Mo}^{1 / 4} \mathrm{~V}}$}


The three CCG tests performed on ferritic $1 / 2 \mathrm{Cr}^{1} / 2 \mathrm{Mo}^{1 / 4} \mathrm{~V}$ steel are summarised in Table 12 . All of these tests were performed at a temperature of $540{ }^{\circ} \mathrm{C}$.

\begin{tabular}{|c|c|c|c|c|c|}
\hline Test ID & $\begin{array}{c}\sigma_{r e f} \\
{[\mathbf{M P a}]}\end{array}$ & $\begin{array}{c}t_{f} \\
{[\mathbf{h}]}\end{array}$ & $\begin{array}{c}t_{0.2}^{A S T M} \\
{[\mathbf{h}]}\end{array}$ & $\begin{array}{l}t_{0.2}^{M O D} \\
{[\mathbf{h}]}\end{array}$ & $\frac{t_{0.2}^{M O D}}{t_{0.2}^{A S T M}}$ \\
\hline 30334_1 & 156 & 3044 & 369 & 560 & 1.5 \\
\hline 30334_3 & 212 & 1007 & 368 & 478 & 1.3 \\
\hline 30334_6 & 232 & 413 & 26 & 174 & 6.6 \\
\hline
\end{tabular}

Table 12: Summary of re-analysed CCG tests performed on $1 / 2 \mathrm{Cr}^{1 / 2} \mathbf{M o} 1 / 4 \mathrm{~V}$.

Again, there is significant variation in the ratio of the times for $0.2 \mathrm{~mm}$ of crack extension to occur based on the modified and ASTM methods. For these three tests, this ratio varies from 1.3 to 6.6. The one with the highest ratio is Test ID 30334_6. For this test, the increase in gradient which is believed to correspond to the onset of crack growth occurs after 137 hours. At this time, if the PD is interpreted in accordance with ASTM E1457, $0.42 \mathrm{~mm}$ of crack extension is predicted due to the accumulation of creep strain.

\section{Discussion}

When performing CCG tests the initial change in PD is often due to the accumulation of creep strain rather than crack growth. Using the current method in ASTM E1457-15 to interpret the data, this change in PD is erroneously interpreted as crack growth and can be a significant source of error. To mitigate this problem a modified method of interpreting the PD data is described below and shown schematically in 


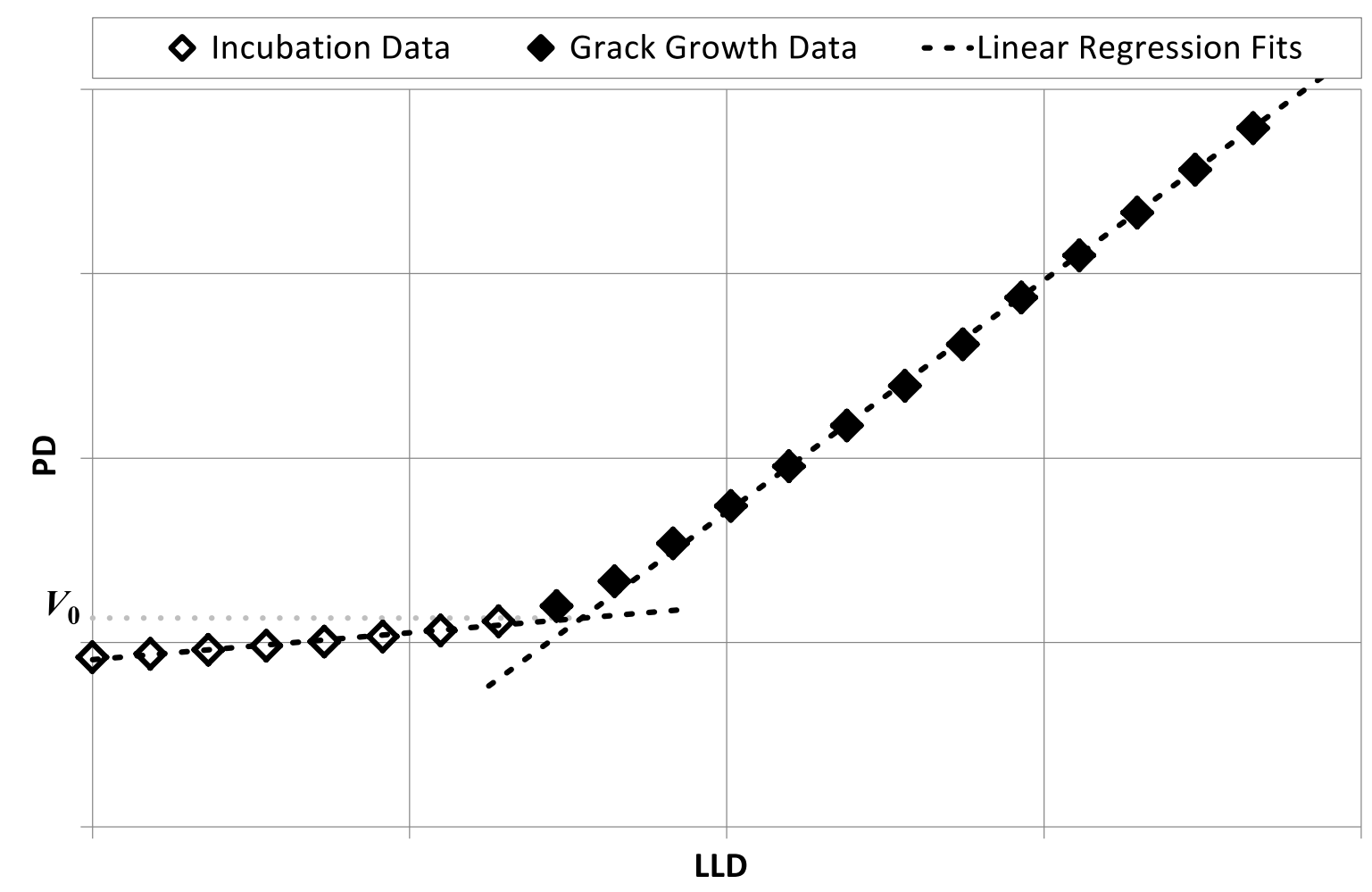

Figure 31. This method is analogous to one used for measuring stable tearing during fracture toughness testing (Lowes and Fearnehough, 1971).

The onset of CCG can be identified from an increase in gradient on a plot of PD (or resistance) against LLD. This increase in gradient has been observed for a range of materials, temperatures and test durations. It corresponds to micro-cracks ahead of the crack tip first linking up with the pre-crack as confirmed by interrupted CCG tests. This experimental observation has been validated by FE analysis.

The exact point of crack initiation can be difficult to identify because the increase in gradient is not abrupt, but occurs over a period of time. This is because, during the very early stages of crack growth, the effects of both strain and crack growth, on PD, are comparable. To estimate the PD corresponding to the onset of $\mathrm{CCG}, V_{0}$, it is proposed that the intersection of linear regressions to the data either side of the increase in gradient should be used, as shown in 


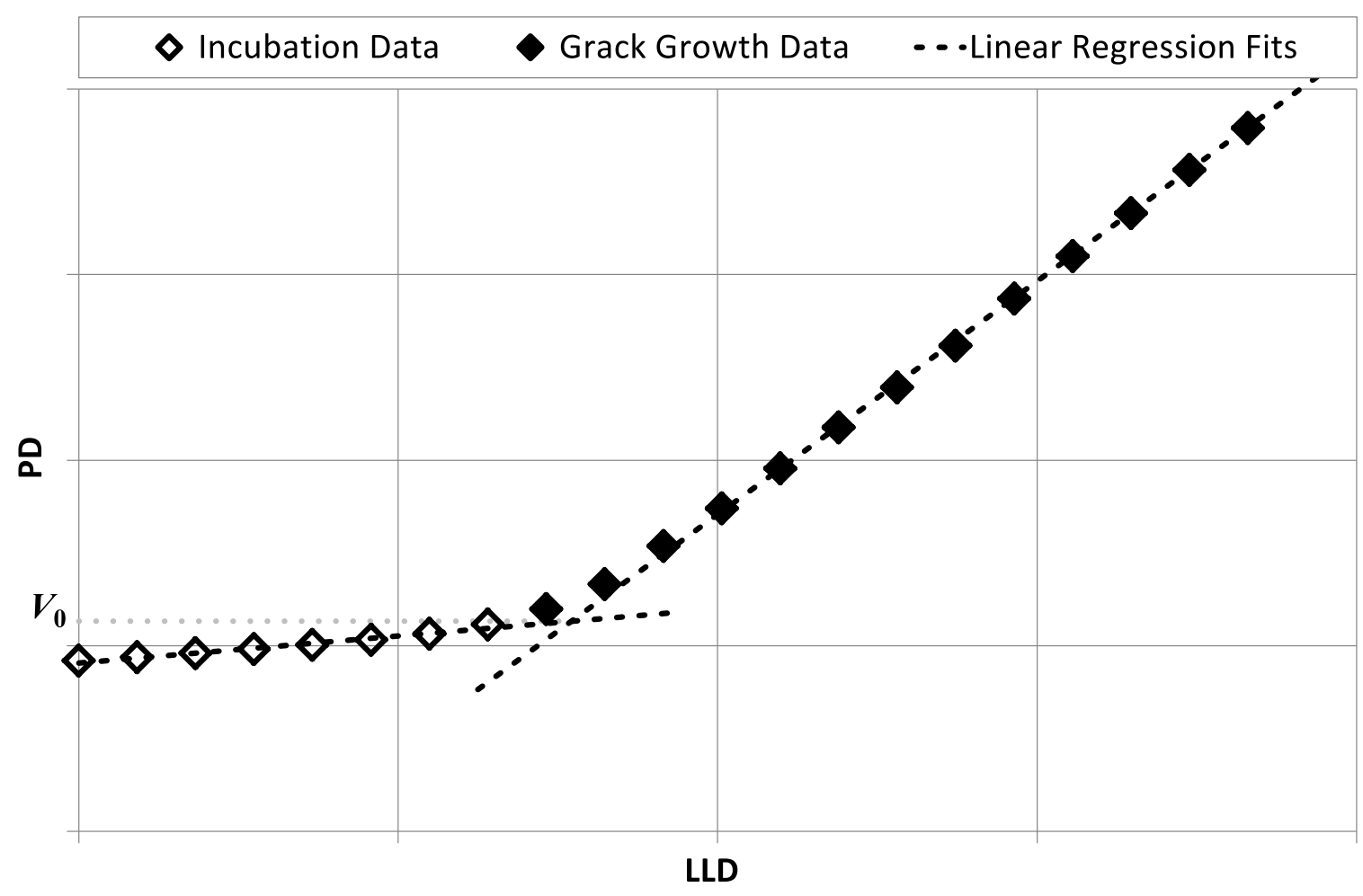

Figure 31 .

For some tests the trends before and/or after the increase in gradient may not be linear. During incubation, a gradual reduction in gradient may be observed due to stress redistribution and/or primary creep effects. An initial drop in PD is also sometimes observed. Furthermore, for tests with large amounts of crack growth, a gradual reduction in gradient after initiation is also likely. Despite these nonlinearities, local quasi-linear regions either side of initiation should still exist so the value of $V_{0}$ may be obtained from the intersection of linear regressions to these regions. Alternatively, suitable non-linear regression fits could be used.

If the instantaneous PD, $V$, is greater than $V_{0}$ then crack growth is assumed to have occurred. The extent of this crack growth can be calculated using a suitable calibration function, such as the one in Equation (1), and the PD values $V$ and $V_{0}$. This approach assumes that the influence of strain on PD is negligible after the onset of crack growth. The validity of this assumption for Type $316 \mathrm{H}$ stainless steel at $550{ }^{\circ} \mathrm{C}$ has been confirmed by $\mathrm{FE}$ analysis. Further analysis is required for materials with higher creep ductility.

Discontinuous cracking and asperities on the fracture surface are common during CCG testing. These can prevent clean separation of the crack faces and provide alternative paths for the current so the PD technique often under-predicts crack extension compared to optical measurements from the post-test fracture surface. The proposed method of interpreting PD 
data does not address this problem. The linear correction in ASTM E1457-15, and provided in Equation (2), is a pragmatic way to account for these discrepancies.

The proposed new method of interpreting PD data has been compared to the current method in ASTM E1457 by re-analysing CCG tests performed on a range of materials, at different temperatures, and for different durations. Significant differences have been identified. The estimated time for $0.2 \mathrm{~mm}$ of crack growth to occur (the engineering definition of crack initiation) was typically between 1 and 2 times longer for the modified method, but for one test the difference was more than a factor of 20 . For this test the spurious crack extension is much greater than $0.2 \mathrm{~mm}$, similar to the preliminary interrupted CCG test performed on P91 steel, and is comparable to the actual crack extension that occurs throughout the test. This can influence the crack growth rates as well as the initiation time such that, in some situations, the ASTM method can be underestimate the crack growth rate by a factor of 1.5. This is non-conservative and potentially dangerous. The proposed new method of interpreting PD data suppresses these errors. It also removes the majority of the so-called 'tail' from the correlation of crack growth rate against $C^{*}$ which demonstrates that, for the materials considered in this study, the tail is primarily due to the erroneous interpretation of creep strains as crack growth.

\section{Conclusions}

When interpreting PD data obtained from CCG testing using the method in ASTM E1457-15, the accumulation of strain during incubation is erroneously interpreted as crack extension. This spurious crack extension can result in crack initiation times that are significantly underestimated by factors of more than 20. Crack initiation models developed from these test results will be excessively conservative and, when used to predict the residual life of real structures, will prompt expensive and unnecessary inspection and maintenance programmes, e.g. the lost revenue associated with the shutdown of a UK Advanced Gas-Cooled Reactor $(\mathrm{AGR})$ is $\sim £ 0.5 \mathrm{~m} /$ day. Furthermore, if the PD measurements are corrected based on the final crack length measured from the post-test fracture surface, which is common, the subsequent crack growth rates can be underestimated resulting in non-conservative CCG rate predictions in real structures.

To mitigate these errors, a novel method of interpreting PD data has been proposed which uses an increase in gradient on a plot of PD against LLD to identify crack initiation (the point at which micro-cracks ahead of the crack tip first link up with the pre-crack). This approach 
supresses any spurious crack extension due to strain that occurs during incubation. It assumes that the influence of strain on PD during subsequent crack growth is negligible. This assumption can be validated using FE analysis. The proposed method also removes the majority of the so-called 'tail' from the correlation of crack growth rate against $C^{*}$. This demonstrates that, for the materials considered in this study, the tail is primarily due to the erroneous interpretation of creep strains as crack growth.

\section{Acknowledgements}

The authors would like to acknowledge the Engineering and Physical Sciences Research Council (EPSRC), UK for the support under grant EP/I004351/1 and the Doctoral Prize Fellowship via The University of Sheffield. This paper is published with permission of EDF Energy Nuclear Generation Ltd.

\section{9. $\quad$ References}

Baker, A., Gladwin, D.N., 2004. Status Report on the 21/4Cr1Mo Weld Metal, 1/2CMV Parent and 1/2CMV HAZ Test Programmes, E/REP/BDBB/0050/AGR/04. British Energy Generation Limited.

Bakker, A., 1985. A DC Potential Drop Procedure for Crack Initiation and R-Curve Measurements During Ductile Fracture Tests. ASTM STP 856 394-410. doi:10.1520/STP34537S

BEGL, 2000. R6 Revision 4: Assessment of the Integrity of Structures Containing Defects.

Bettinson, A.D., 2001. The Influence of Constraint on the Creep Crack Growth of 316H Stainless Steel. Department of Mechanical Engineering, Imperial College, London.

COMSOL, 2012. Multiphysics v4.3a. Comsol Ltd., Cambridge, UK.

Dean, D.W., Gladwin, D.N., 2004. Creep Crack Incubation and Growth Behaviour of Type 316H Steels, E/REP/BDBB/0040/GEN/03. British Energy Generation Limited.

Fessler, E., Andrieu, E., Bonnand, V., Chiaruttini, V., Pierret, S., 2017. Relation between crack growth behaviour and crack front morphology under hold-time conditions in DA Inconel 718. Int. J. Fatigue 96, 17-27.

Freeman, B.L., Neate, G.J., 1980. The Measurement of Crack Length During Fracture at Elevated Temperatures Using the D. C. Potential Drop Technique, in: Beevers, C.J. (Ed.), The Measurement of Crack Length and Shape During Fracture and Fatigue. Engineering 
Materials Advisory Services Ltd.

Gladwin, D.N., 1998. The Creep Crack Growth Behaviour of Esshette 1250 Parent, HAZ and Weld, EPD/GEN/REP/0290/98. Nuclear Electric Limited.

Haigh, J.R., 1975. The Mechanisms of Macroscopic High Temperature Crack Growth Part I: Experiments on Tempered Cr-Mo-V Steels. Mater. Sci. Eng. 20, 213-223.

Houlton, N., 2013. Life Extension of the EDF Energy Nuclear Fleet.

Ke, Y., Ståhle, P., 1993. Crack Length Measurements with a Potential Drop Method: A Finite Element Simulation. Int. J. Numer. Methods Eng. 36, 3205-3220. doi:10.1002/nme.1620361809

Ljustell, P., 2011. The Effect of Large Scale Plastic Deformation on Fatigue Crack Length Measurement with the Potential Drop Method. J. Test. Eval. 39, 985-1002. doi:10.1520/JTE103532

Lowes, J.M., Fearnehough, G.D., 1971. The Detection of Slow Crack Growth in Crack Opening Displacement Specimens Using an Electrical Potential Method. Eng. Fract. Mech. 3, 103-104. doi:10.1016/0013-7944(71)90002-6

Madhi, E., Nagy, P.B., 2011. Sensitivity Analysis of a Directional Potential Drop Sensor for Creep Monitoring. NDT E Int. 44, 708-717. doi:10.1016/j.ndteint.2011.08.001

McIntyre, P., Priest, A.H., 1971. Measurement of Sub-Critical Flaw Growth in Stress Corrosion, Cyclic Loading and High Temperature Creep by the DC Electrical Resistance Technique, Report: MG/54/71.

Mehmanparast, A., 2012. The Influence of Inelastic Damage on Creep, Fatigue and Fracture Toughness. Ph.D. Thesis, Department of Mechanical Engineering, Imperial College, London.

Merah, N., 2003. Detecting and Measuring Flaws Using Electric Potential Techniques. J. Qual. Maint. Eng. 9, 160-175.

Merah, N., Bui-Quoc, T., Bernard, M., 1995. Calibration of DC Potential Technique using an Optical Image Processing System in LCF Testing. J. Test. Eval. 23, 160-167.

NIH, 2013. ImageJ v1.48.

NIMS, 2014. Creep Datasheet 43A: Datasheet on the Elevated Temperature Properties of 9Cr1Mo-V-Nb Steel Seemless Pipe for High Temperature Service. National Institute for 
Materials Science.

Ofgem, 2016. Wholesale Energy Markets in 2016.

Orlova, A., Buršık, J., Kuchařová, K., Sklenička, V., 1998. Microstructural Development During High Temperature Creep of 9\% Cr steel. Mater. Sci. Eng. A 245, 39-48.

Panait, C.G., Zielińska-Lipiec, A., Koziel, T., Czyrska-Filemonowicz, A., Gourgues-Lorenzon, A., Bendick, W., 2010. Evolution of Dislocation Density, Size of Subgrains and MX-Type Precipitates in a P91 Steel During Creep and During Thermal Ageing at $600 \mathrm{C}$ for more than 100,000 h. Mater. Sci. Eng. A 527, 4062-4069.

RCC-MR, 2002. Appendix 16: Guide for Leak Before Break Analysis and Defect Assessment.

Riemelmoser, F.O., Weinhandl, H., Kolednik, O., Pippan, R., 1999. The influence of irregularities in the crack shape on the crack extension measurement by means of the direct-current-potential-drop method. J. Test. Eval. 27, 42-46.

Saxena, A., 1980. Electrical Potential Technique for Monitoring Subcritical Crack Growth at Elevated Temperatures. Eng. Fract. Mech. 13, 741-750. doi:10.1016/00137944(80)90005-3

Tarnowski, K.M., Davies, C.M., Dean, D.W., Nikbin, K.M., 2017. Predicting the Influence of Strain on Crack Length Measurements Performed using the Potential Drop Method, Submitted to: Eng. Fract. Mech.

Tarnowski, K.M., Davies, C.M., Dean, D.W., Nikbin, K.M., 2014. The Influence of Plasticity on Crack Length Measurements Using the Potential Drop Technique. ASTM STP 1584 73-96. doi:10.1520/STP158420140055

Webster, G.A., Ainsworth, R.A., 1994. High temperature Component Life Assessment. Chapman and Hall.

Webster, G.A., Davies, C.M., Skelton, R.P., Nikbin, K.M., 2008. Comparison of R5 Volume 4/5 Procedures for Determining Creep-Crack Incubation Periods in Type 316H Stainless Steel. I.C. Consultants.

Wilkowski, G.M., Maxey, W.A., 1983. Review and Applications of the Electronic Potential Method for Measuring Crack Growth in Specimens, Flawed Pipes and Pressure Vessels. ASTM STP 791 2, 266-294. 


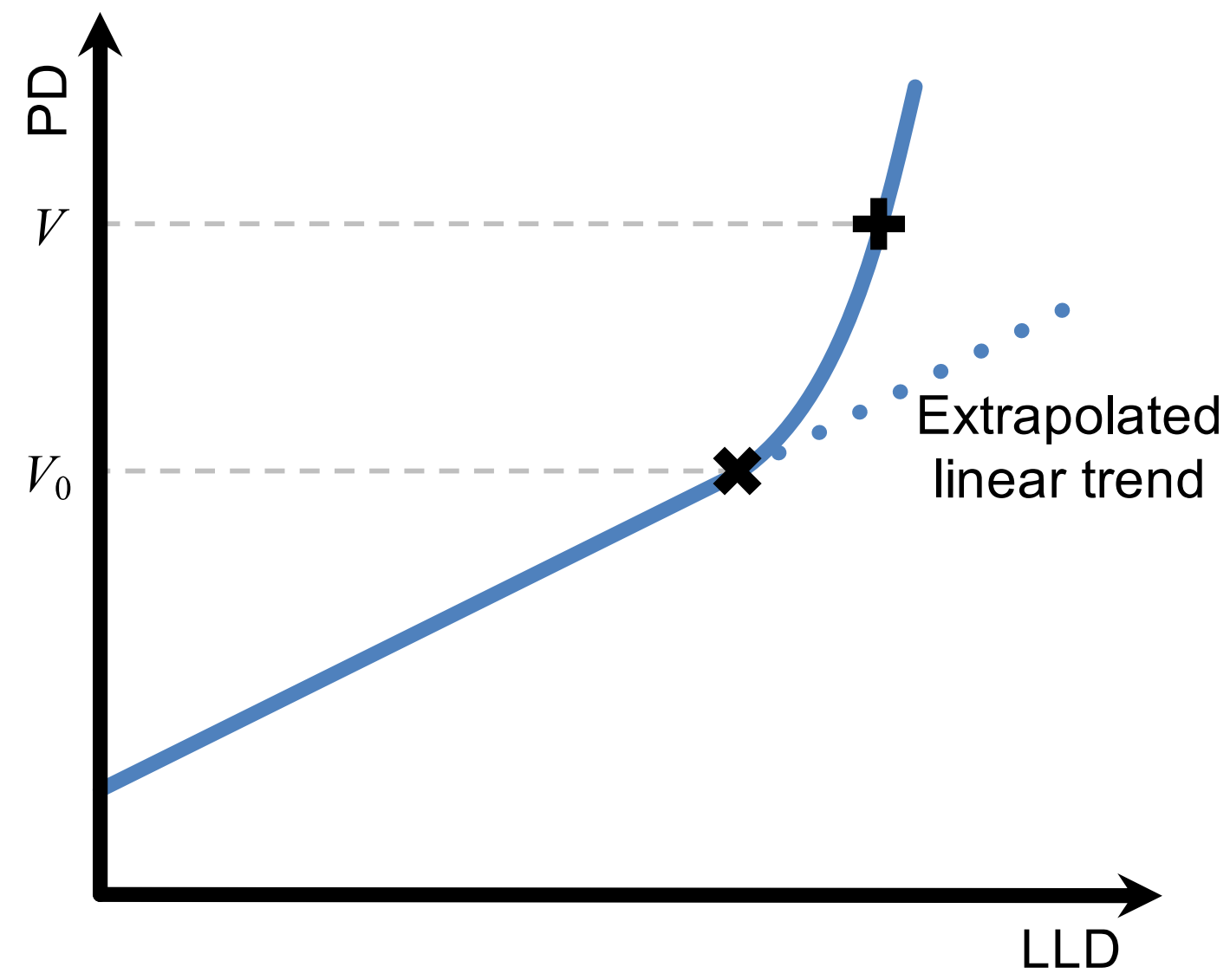

Figure 1: Interpretation of PD data during a fracture toughness test. The point of crack initiation is denoted by ' $x$ ' and the PD values use to calculate crack extension at ' + ' are shown.
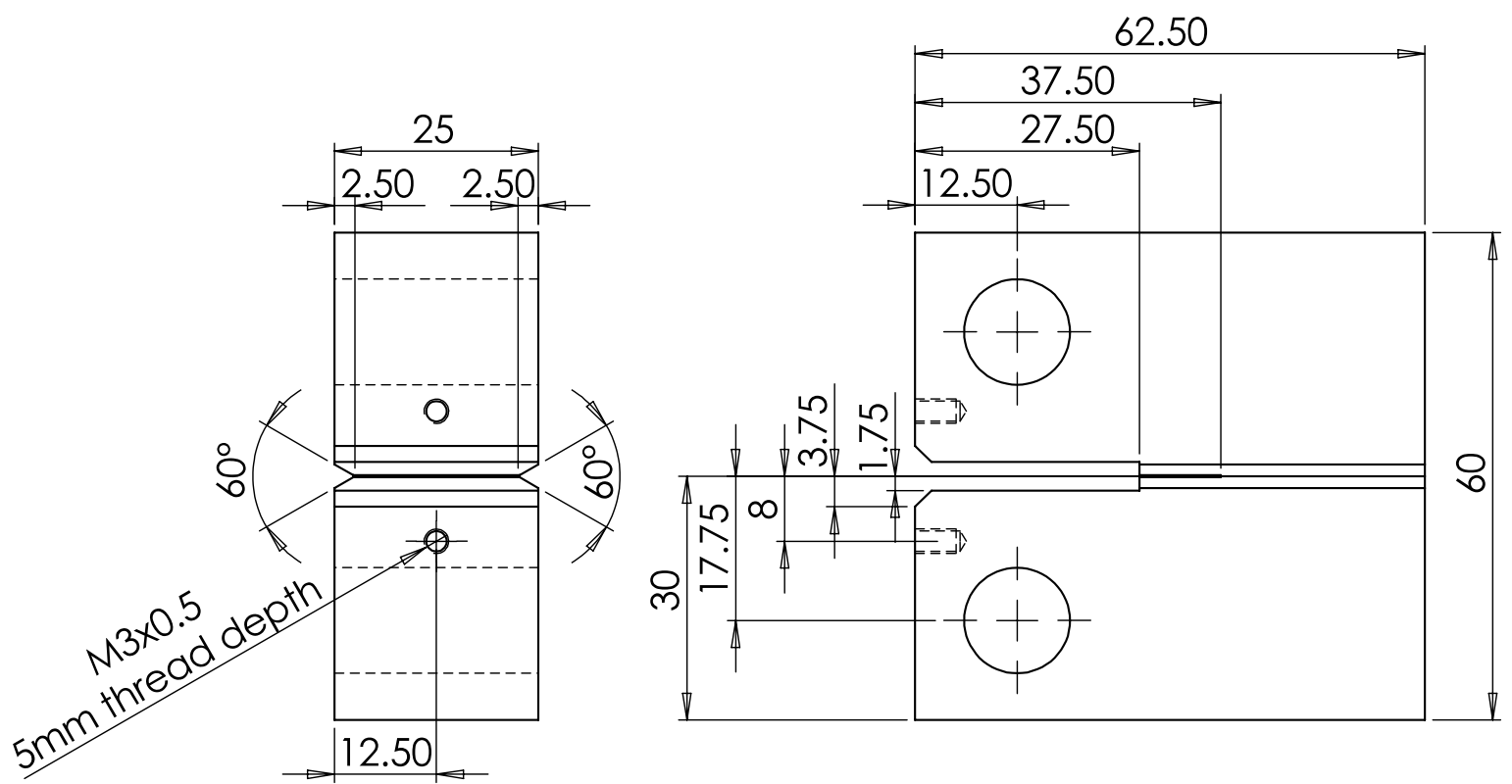

Figure 2: C(T) specimen geometry. 


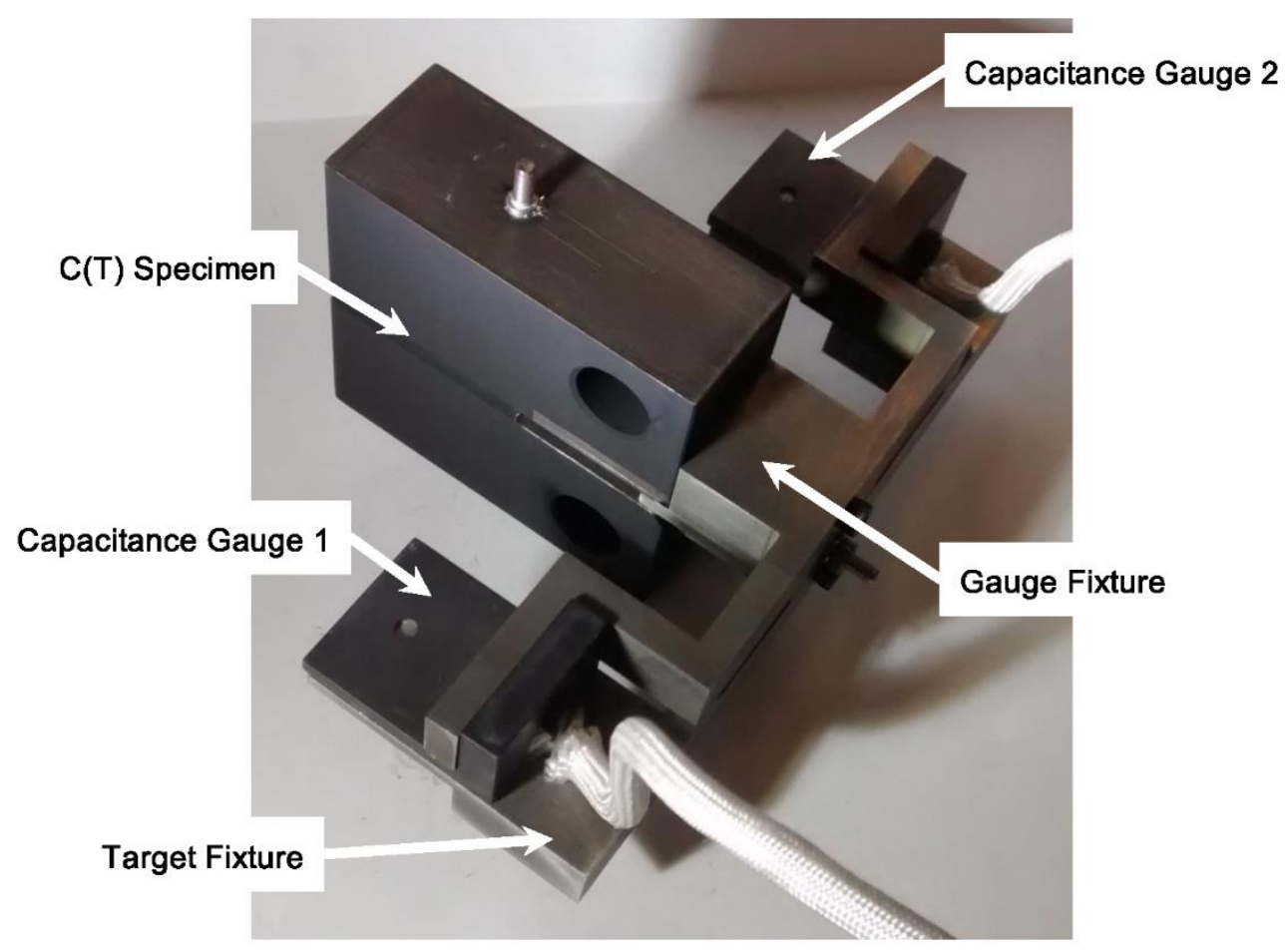

Figure 3: Fixture used to measure LLD for the CCG test performed on P91.

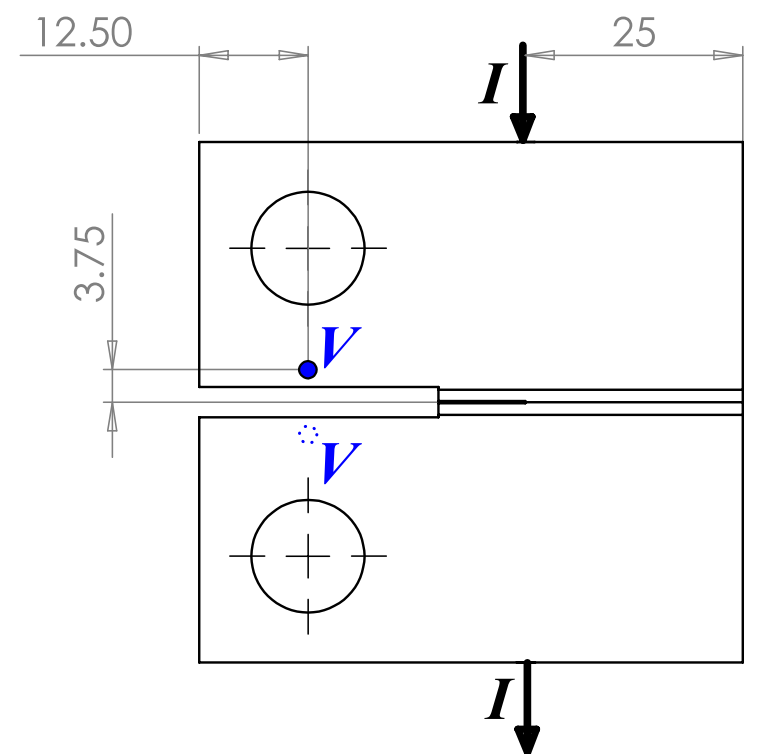

(a)

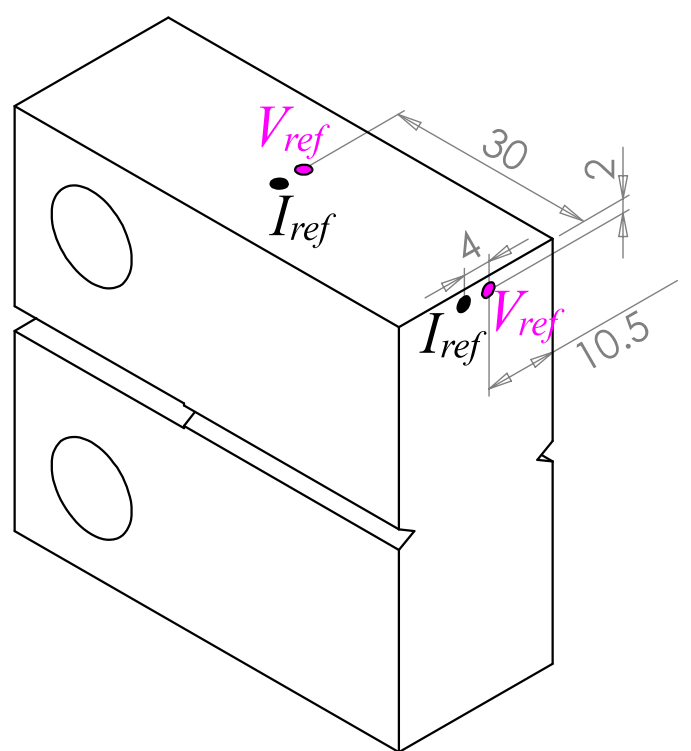

(b)

Figure 4: PD Configuration for (a) the crack length measurement, and (b) the reference measurement. 


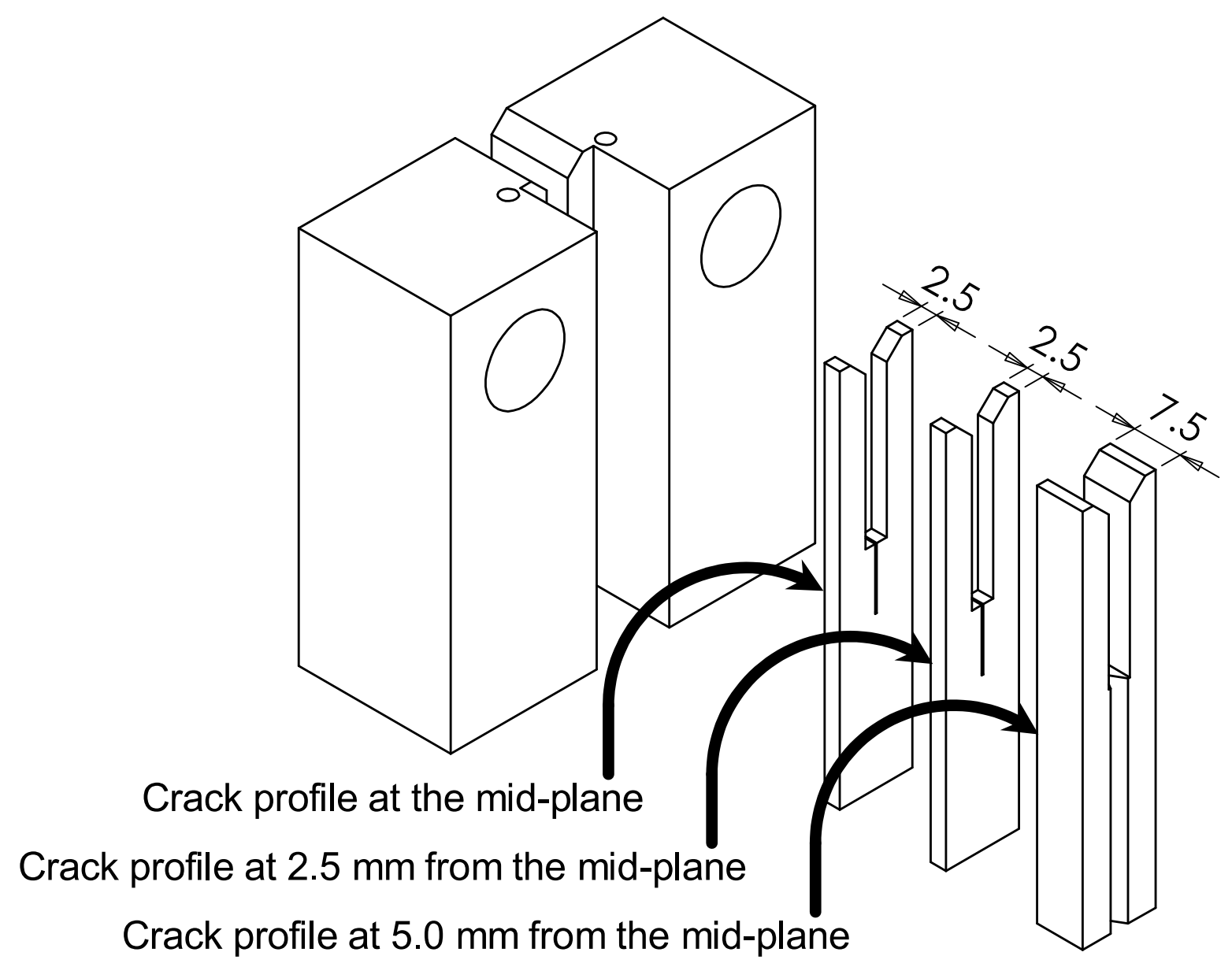

Figure 5: Post-test sectioning to reveal the crack profile at the mid-plane and at $2.5 \mathrm{~mm}$ and $5.0 \mathrm{~mm}$ from the mid-plane.
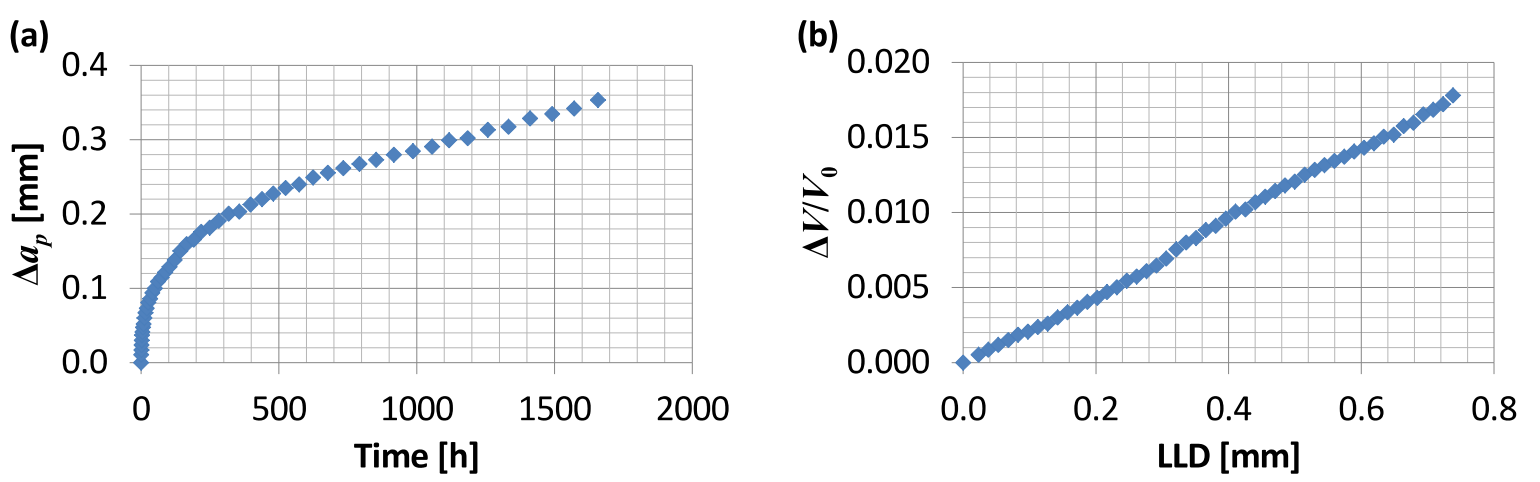

Figure 6: Results of the P91 CCG test showing (a) the crack extension predicted from the PD, and (b) the relationship between relative change in PD and LLD. 


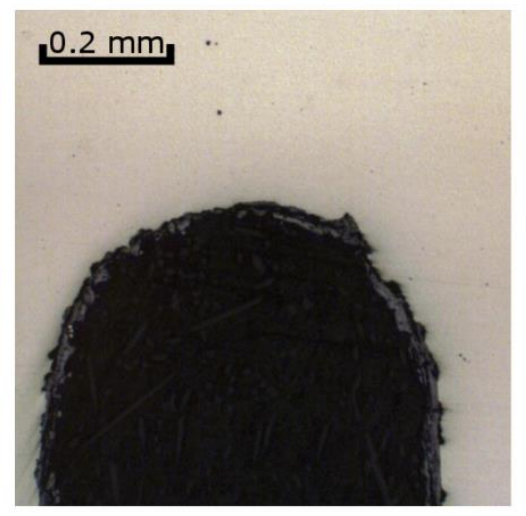

(a)

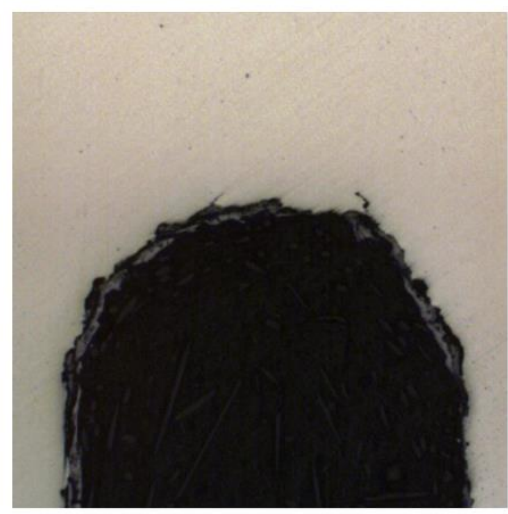

(b)

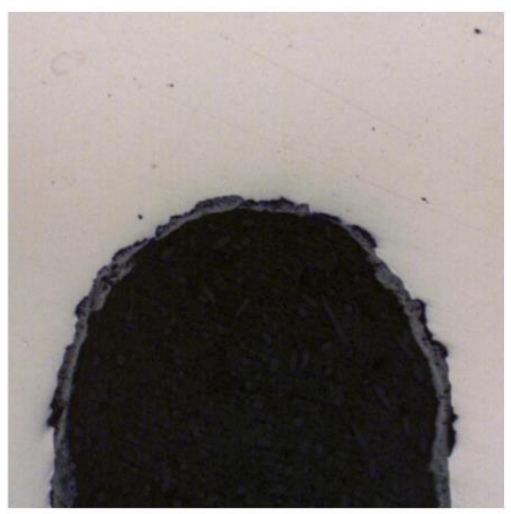

(c)

Figure 7: Crack tip profile from the P91 CCG test performed at (a) the mid-plane, (b) $2.5 \mathrm{~mm}$ from the mid-plane, and (c) $5.0 \mathrm{~mm}$ from the mid-plane.

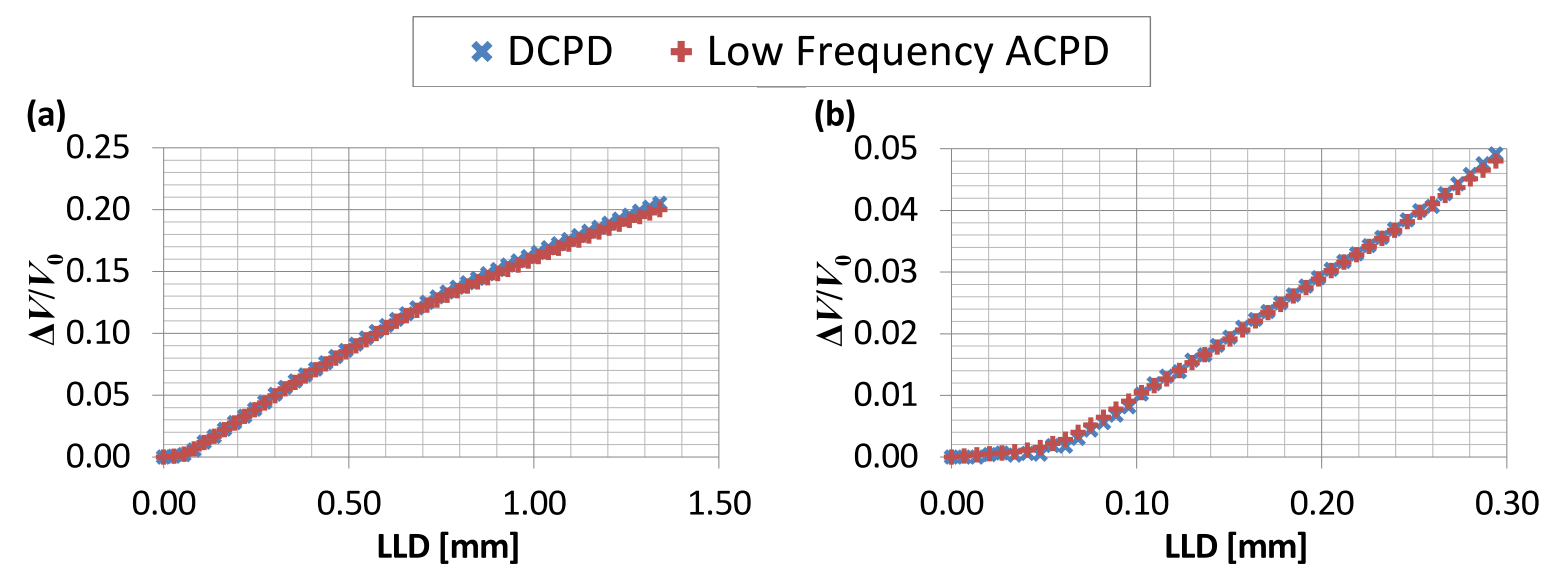

Figure 8: Relative change in PD vs. LLD during a CCG test, comparing the response of DCPD and low frequency ACPD for (a) the full duration of the test, and (b) the initial part of the test.

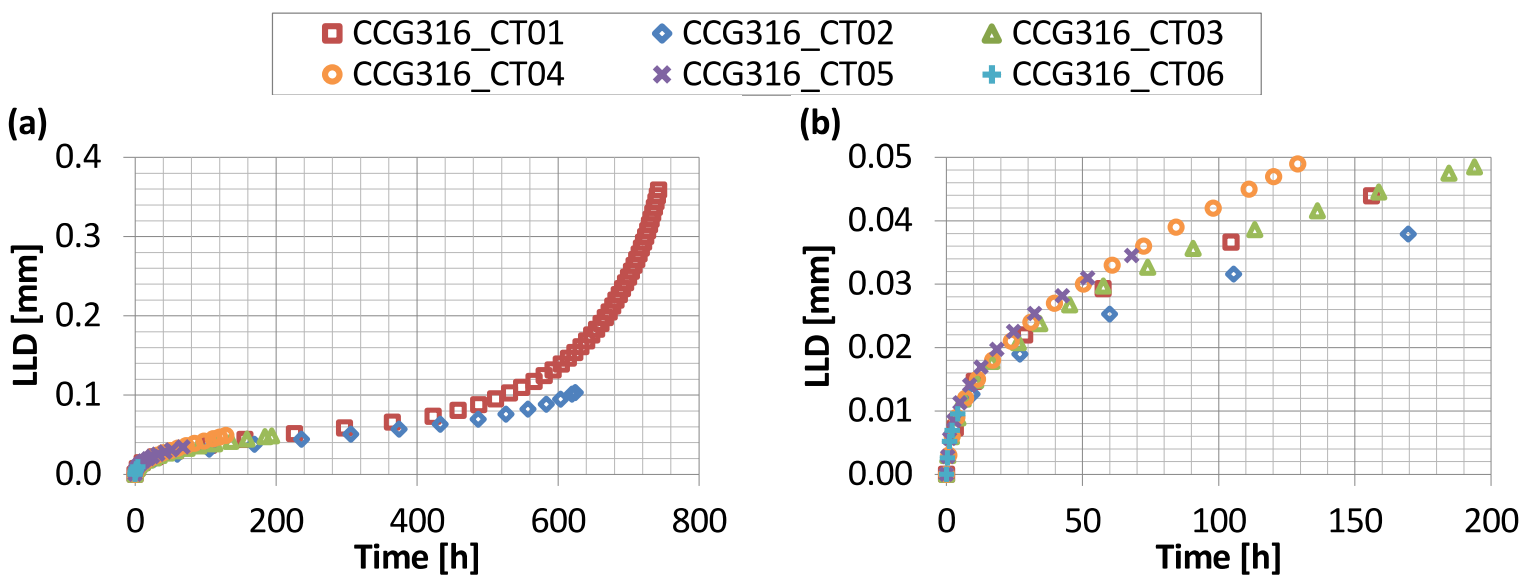

Figure 9: LLD for the interrupted CCG tests showing (a) the entire test, and (b) the initial 200 hours. 


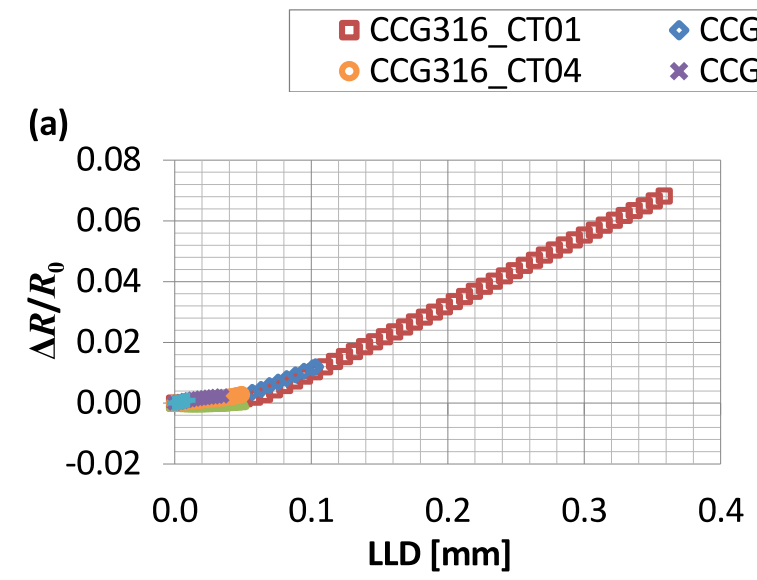

$\begin{array}{ll}\text { CT02 } & \triangle \text { CCG316_CT03 } \\ \text { CT05 } & + \text { CCG316_CT06 }\end{array}$

(b)

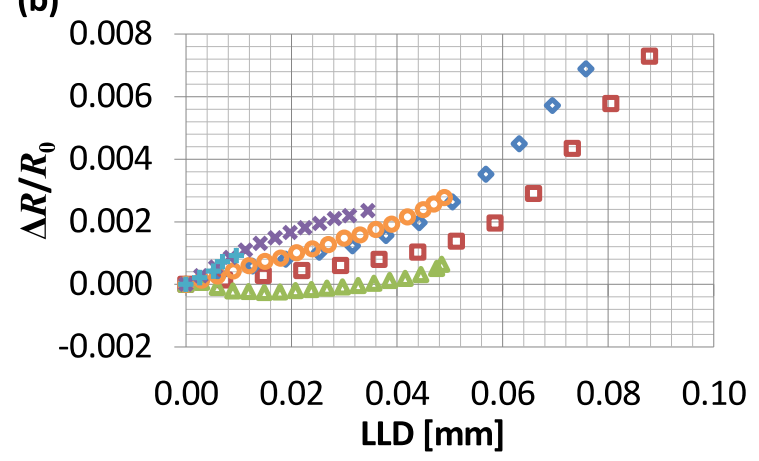

Figure 10: Relative change in resistance vs. LLD for the interrupted CCG tests showing (a) all data, and (b) the early stages of each test. 

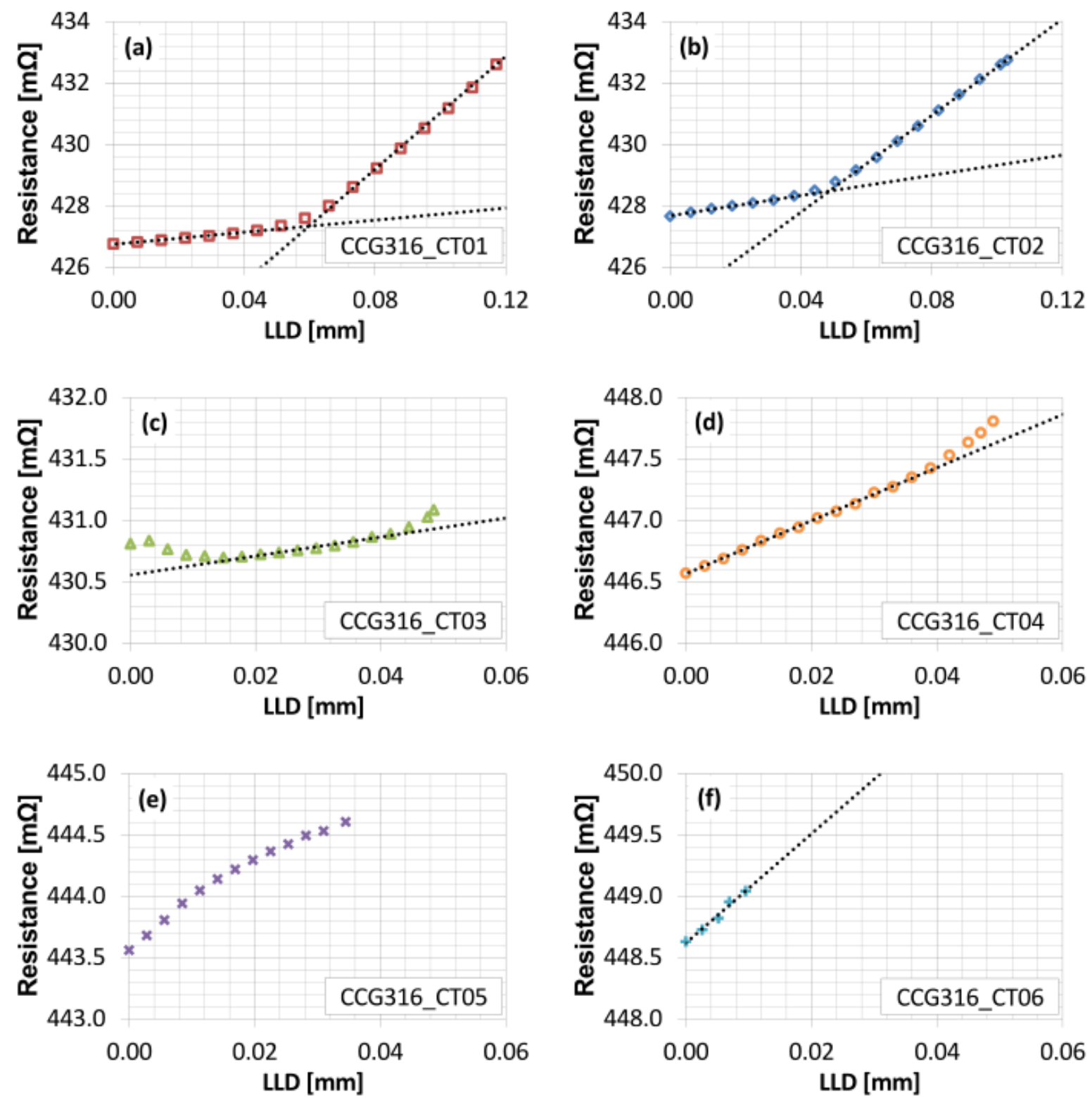

Figure 11: Resistance vs. LLD during the early stages of each interrupted CCG tests. 


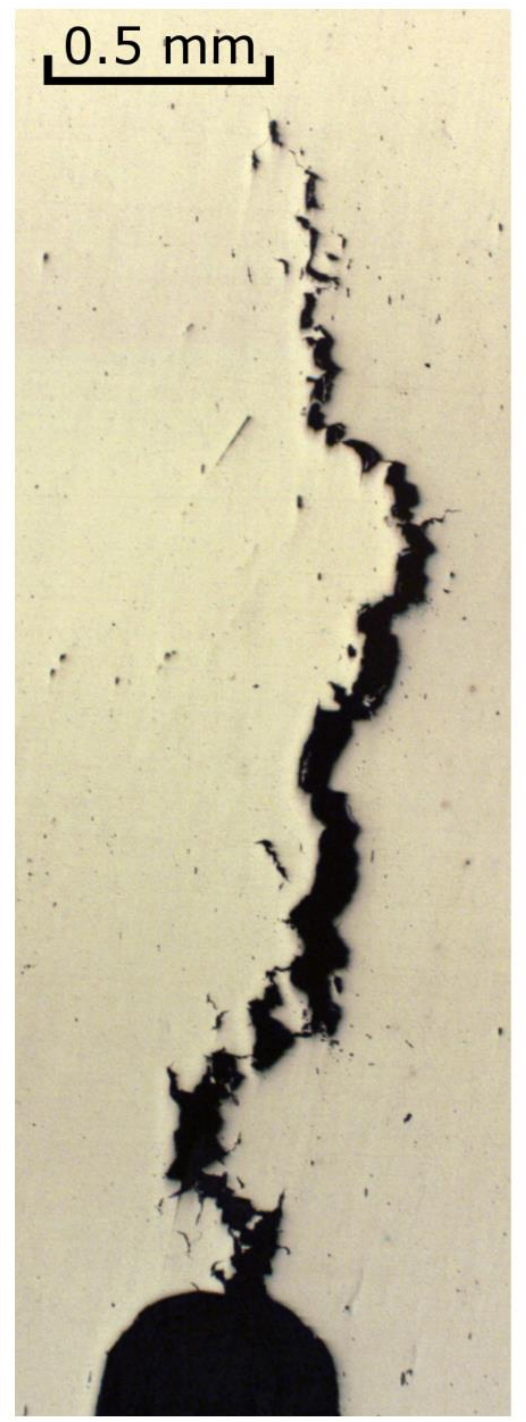

(a)

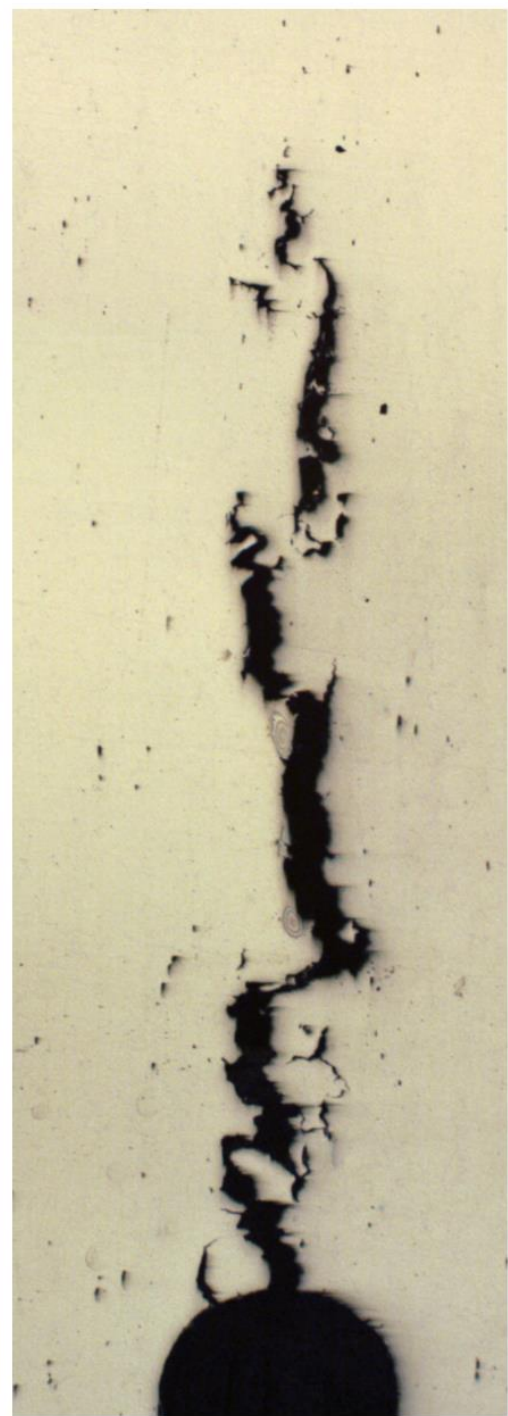

(b)

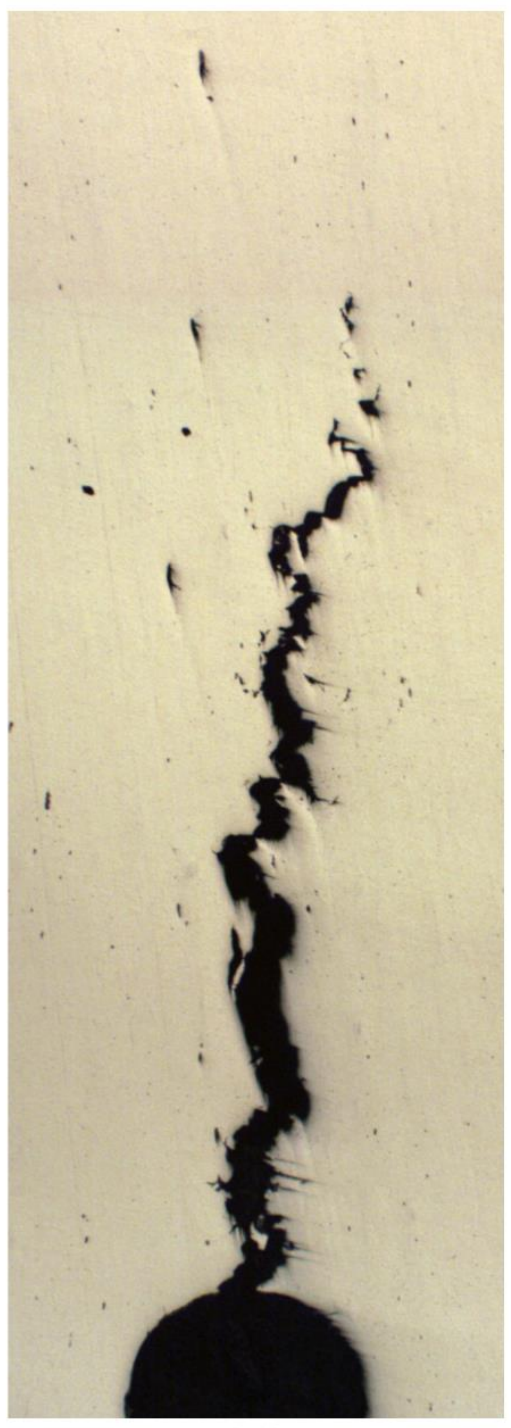

(c)

Figure 12: Crack profiles for specimen CCG316_CT01 at (a) the mid-plane, (b) $2.5 \mathrm{~mm}$ from the mid-plane, and (c) $5.0 \mathrm{~mm}$ from the mid-plane. 


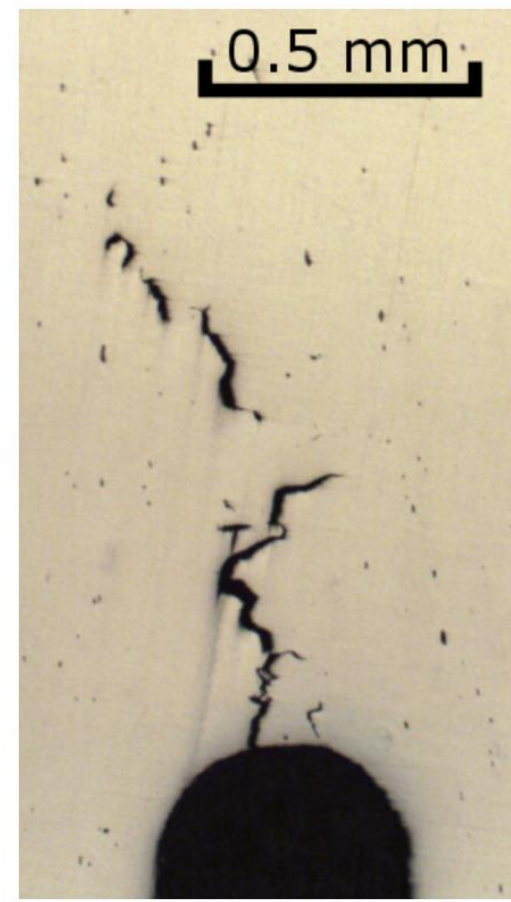

(a)

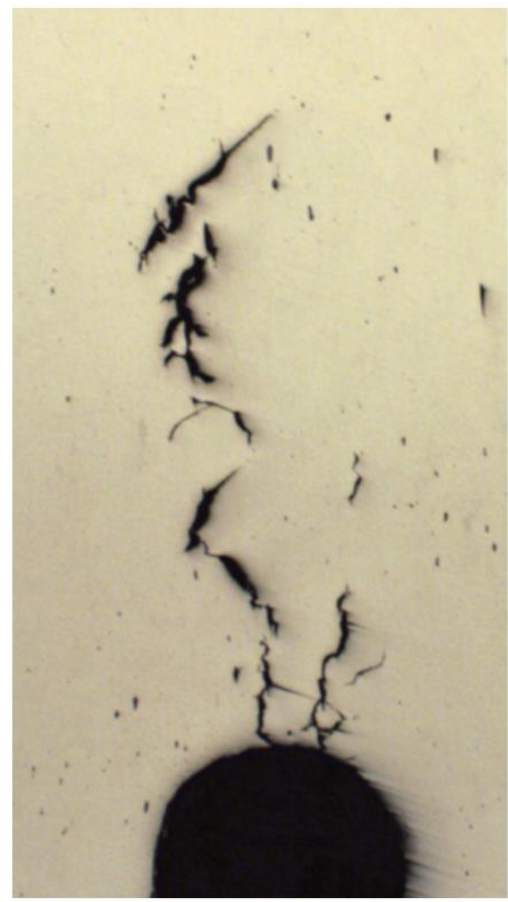

(b)

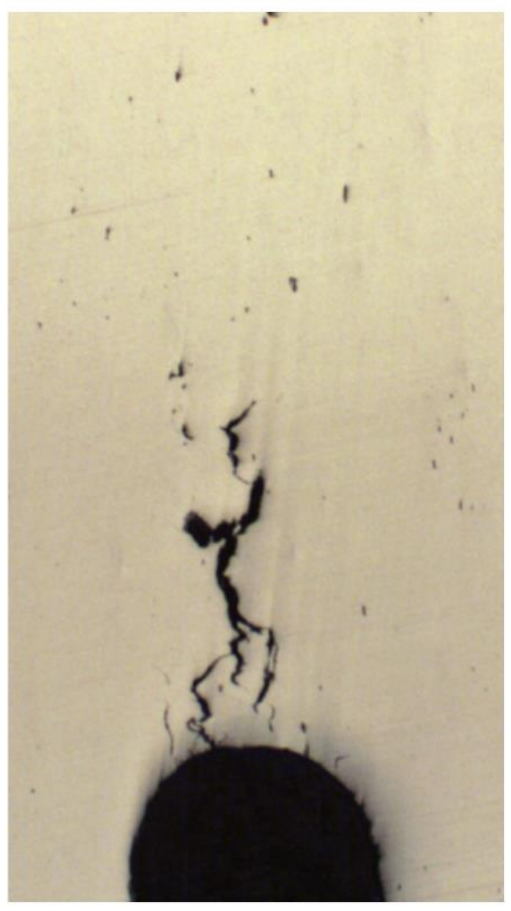

(c)

Figure 13: Crack profiles for specimen CCG316_CT02 at (a) the mid-plane, (b) $2.5 \mathrm{~mm}$ from the mid-plane, and (c) $5.0 \mathrm{~mm}$ from the mid-plane.

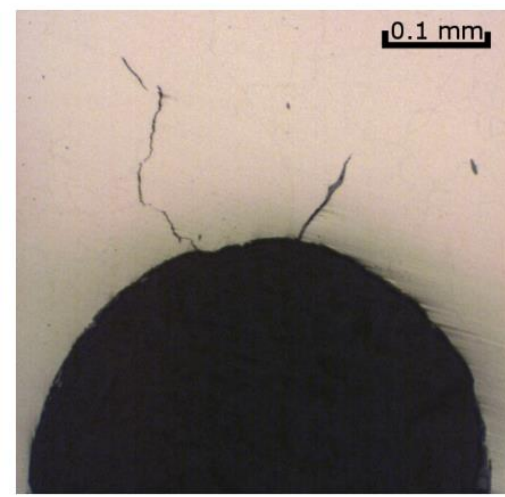

(a)

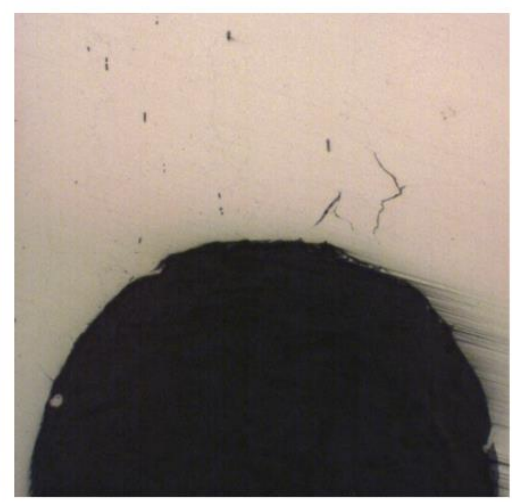

(b)

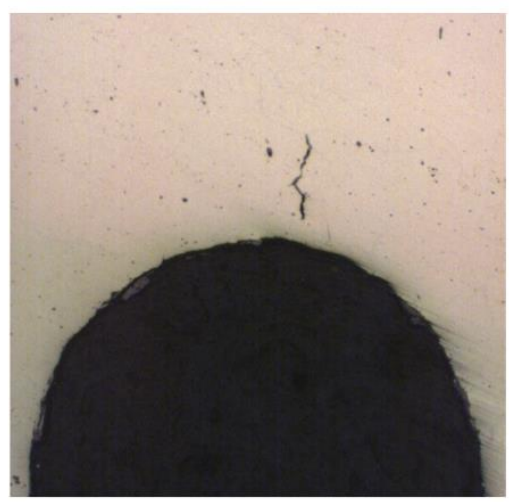

(c)

Figure 14: Crack profiles for specimen CCG316_CT03 at (a) the mid-plane, (b) $2.5 \mathrm{~mm}$ from the mid-plane, and (c) $5.0 \mathrm{~mm}$ from the mid-plane. 


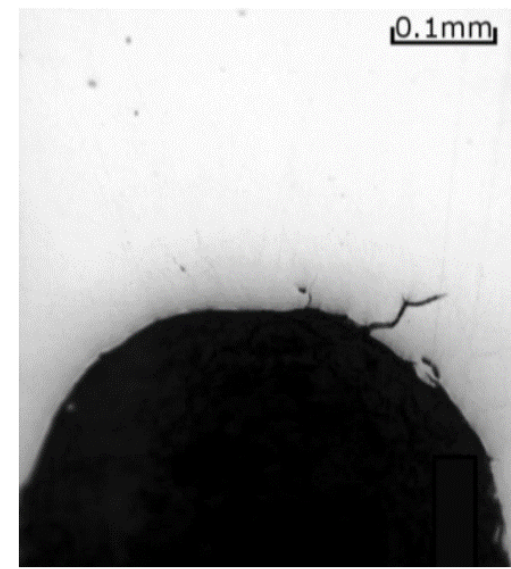

(a)

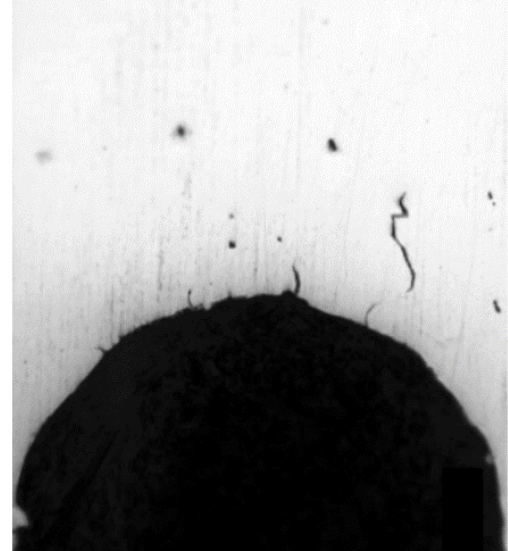

(b)

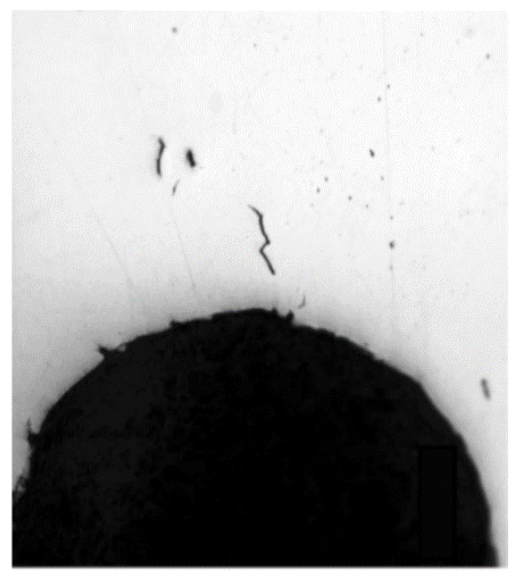

(c)

Figure 15: Crack profiles for specimen CCG316_CT04 at (a) the mid-plane, (b) $2.5 \mathrm{~mm}$ from the mid-plane, and (c) $5.0 \mathrm{~mm}$ from the mid-plane.

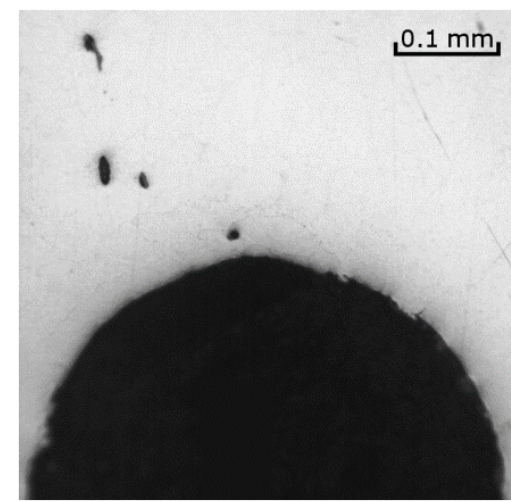

(a)

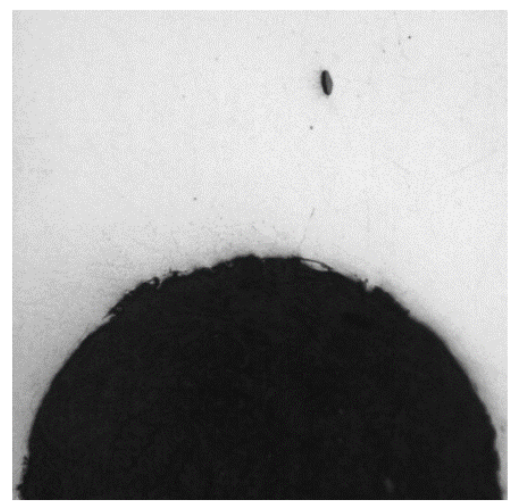

(b)

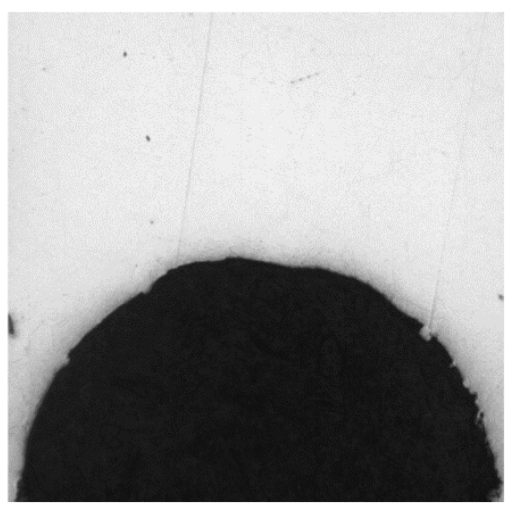

(c)

Figure 16: Crack profile of specimen CCG316_CT05 at (a) the mid-plane, (b) $2.5 \mathrm{~mm}$ from the mid-plane, and (c) $5.0 \mathrm{~mm}$ from the mid-plane.

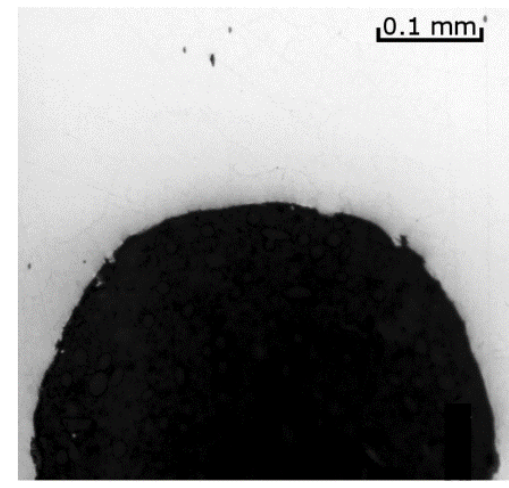

(a)

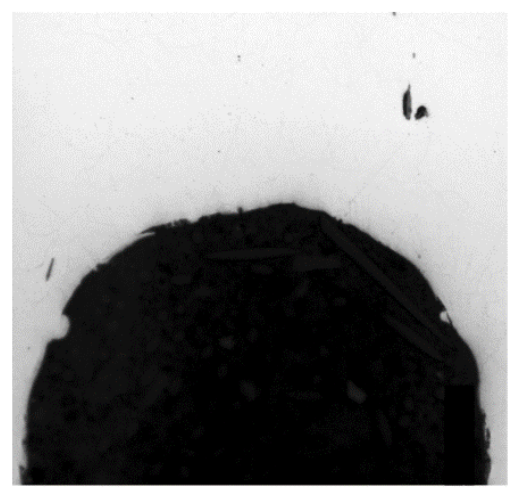

(b)

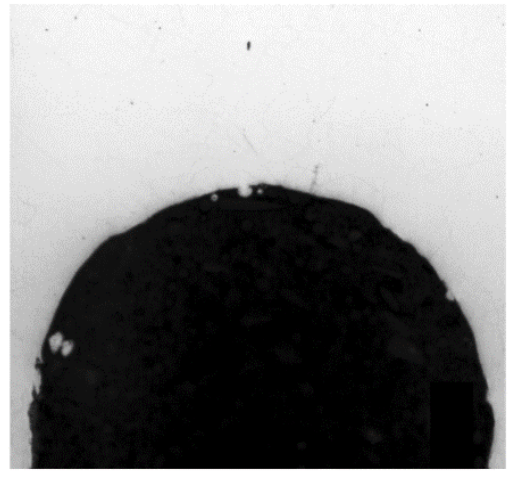

(c)

Figure 17: Crack profiles for specimen CCG316_CT06 at (a) the mid-plane, (b) $2.5 \mathrm{~mm}$ from the mid-plane, and (c) $5.0 \mathrm{~mm}$ from the mid-plane. 


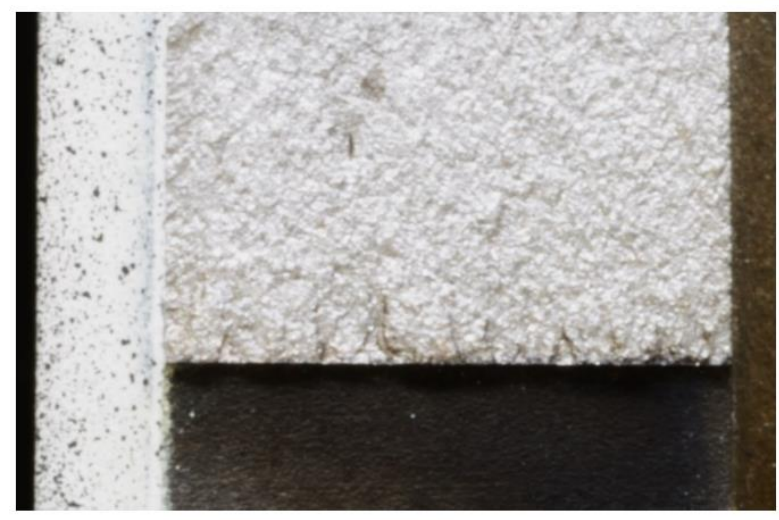

(a)

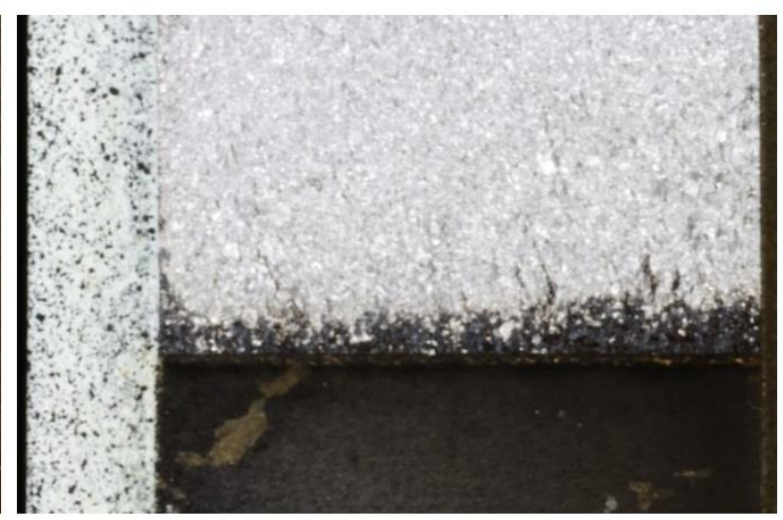

(b)

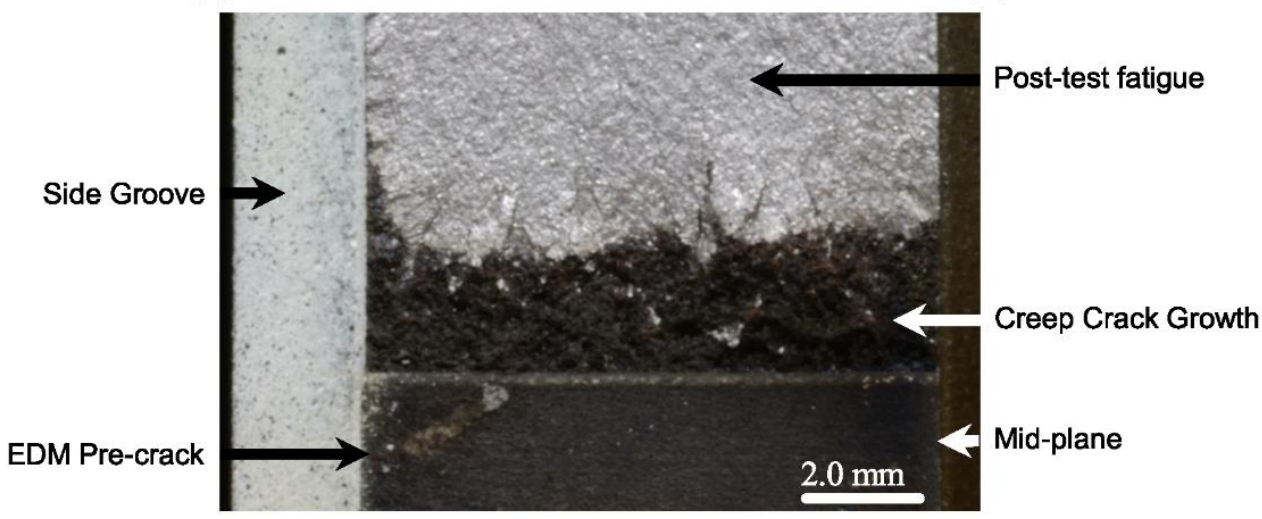

(c)

Figure 18: Fracture surface of specimens (a) CCG316_CT03, (b) CCG316_CT02, and (c) CCG316_CT01.

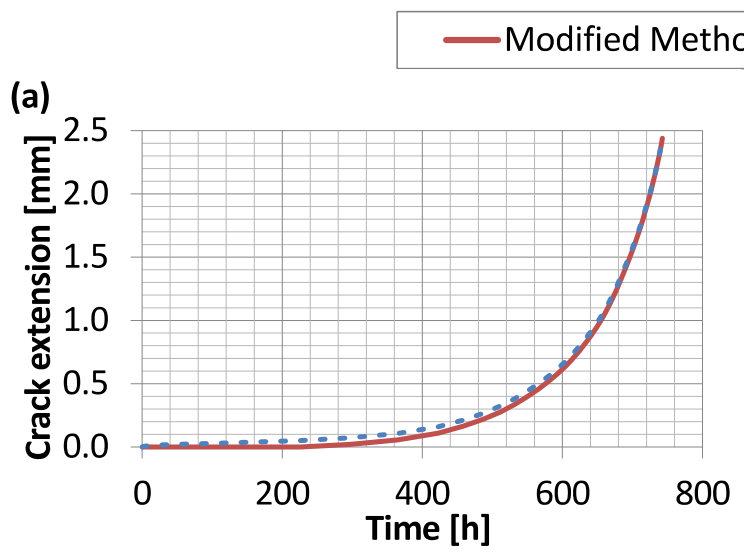

(b)

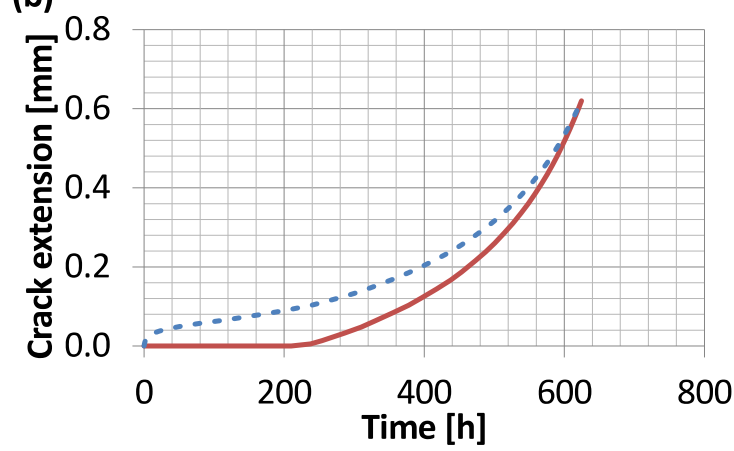

Figure 19: Corrected crack extension for (a) CCG316_CT01, and (b) CCG316_CT02. 

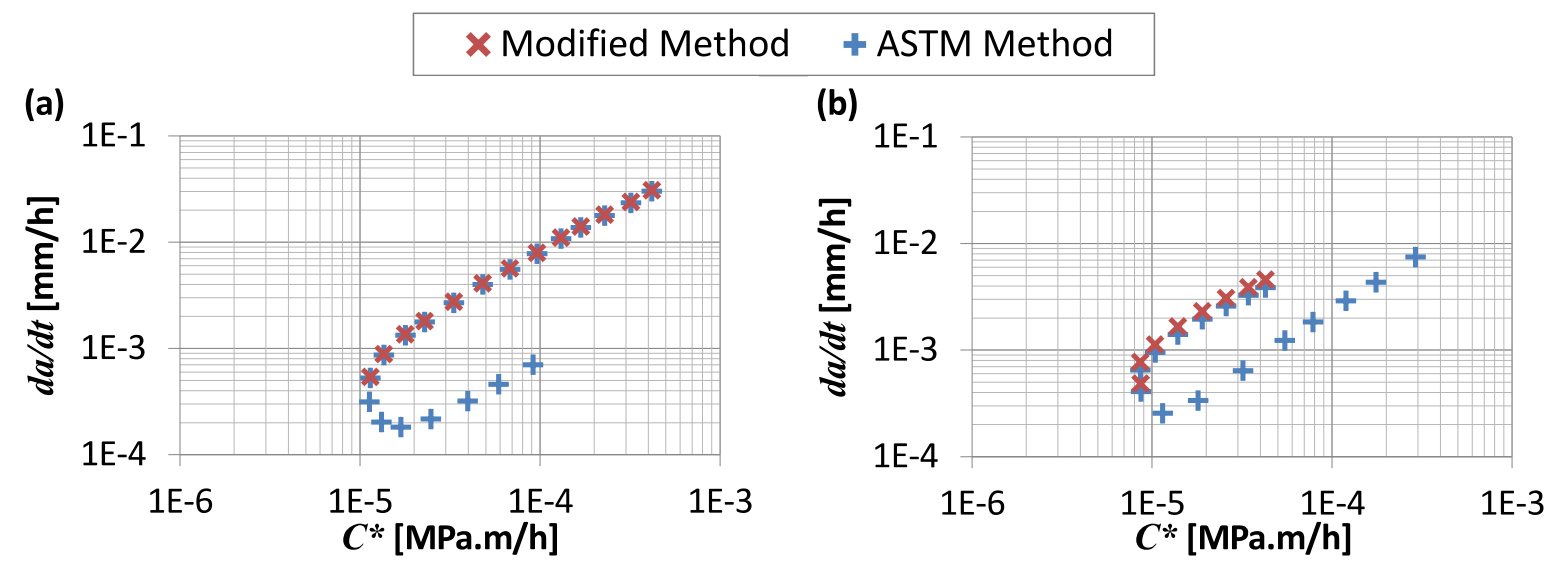

Figure 20: Correlation between crack growth rate and $C^{*}$ for (a) CCG316_CT01, and (b) CCG316_CT02.

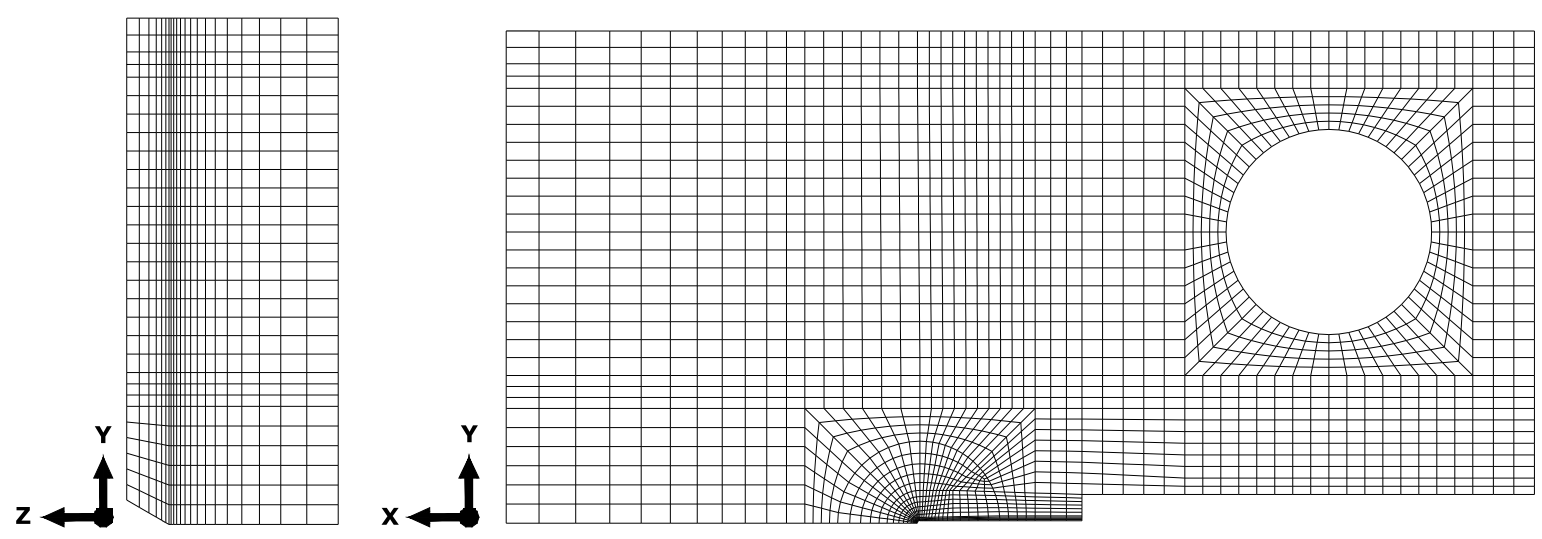

Figure 21: FE model 'CCG_CI' used to predict the change in PD up to crack initiation.

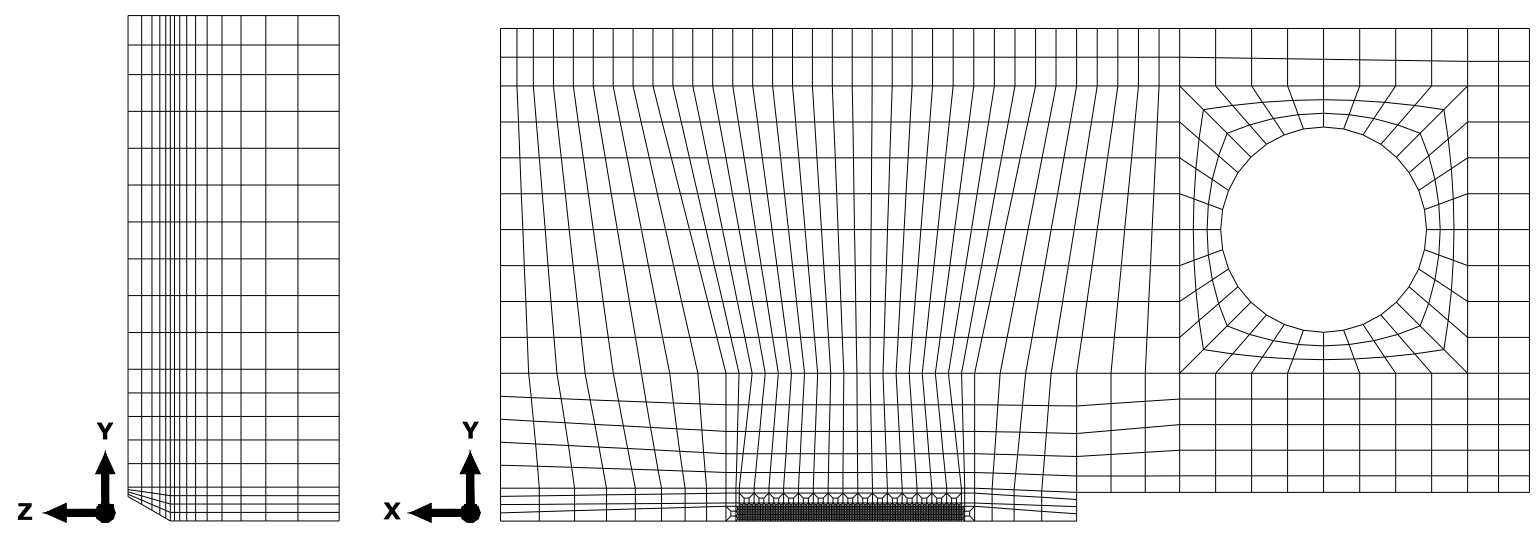

Figure 22: FE model 'CCG_CG' used to predict the change in PD including crack growth. 


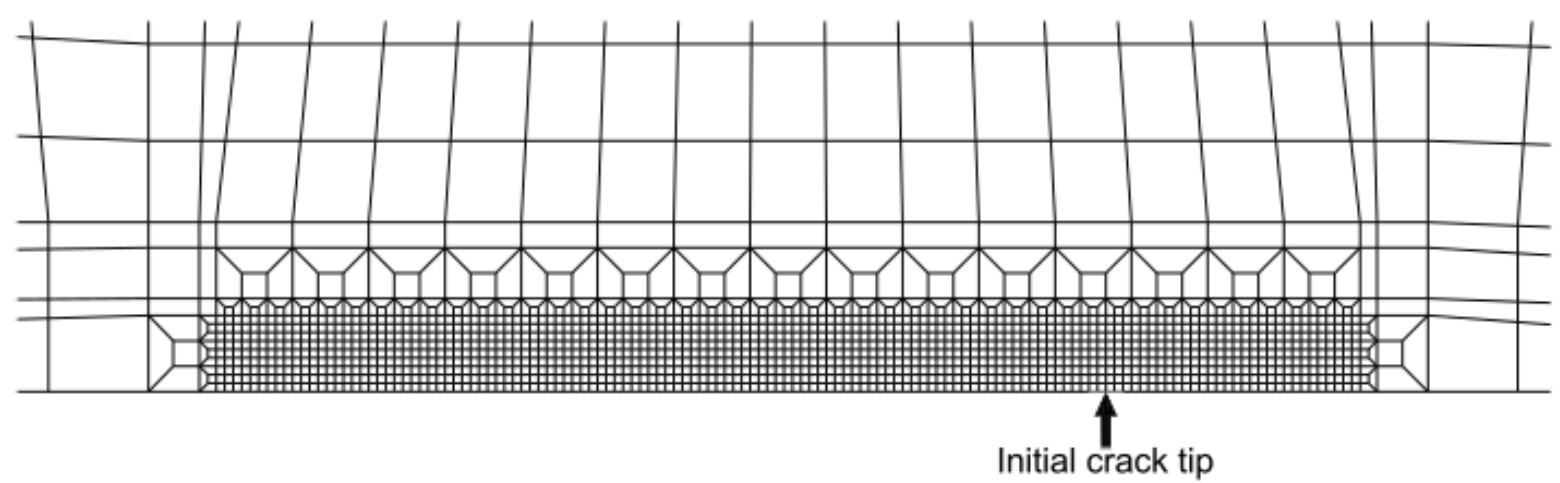

Figure 23: Refined crack tip mesh in model 'CCG_CG' for modelling crack growth.

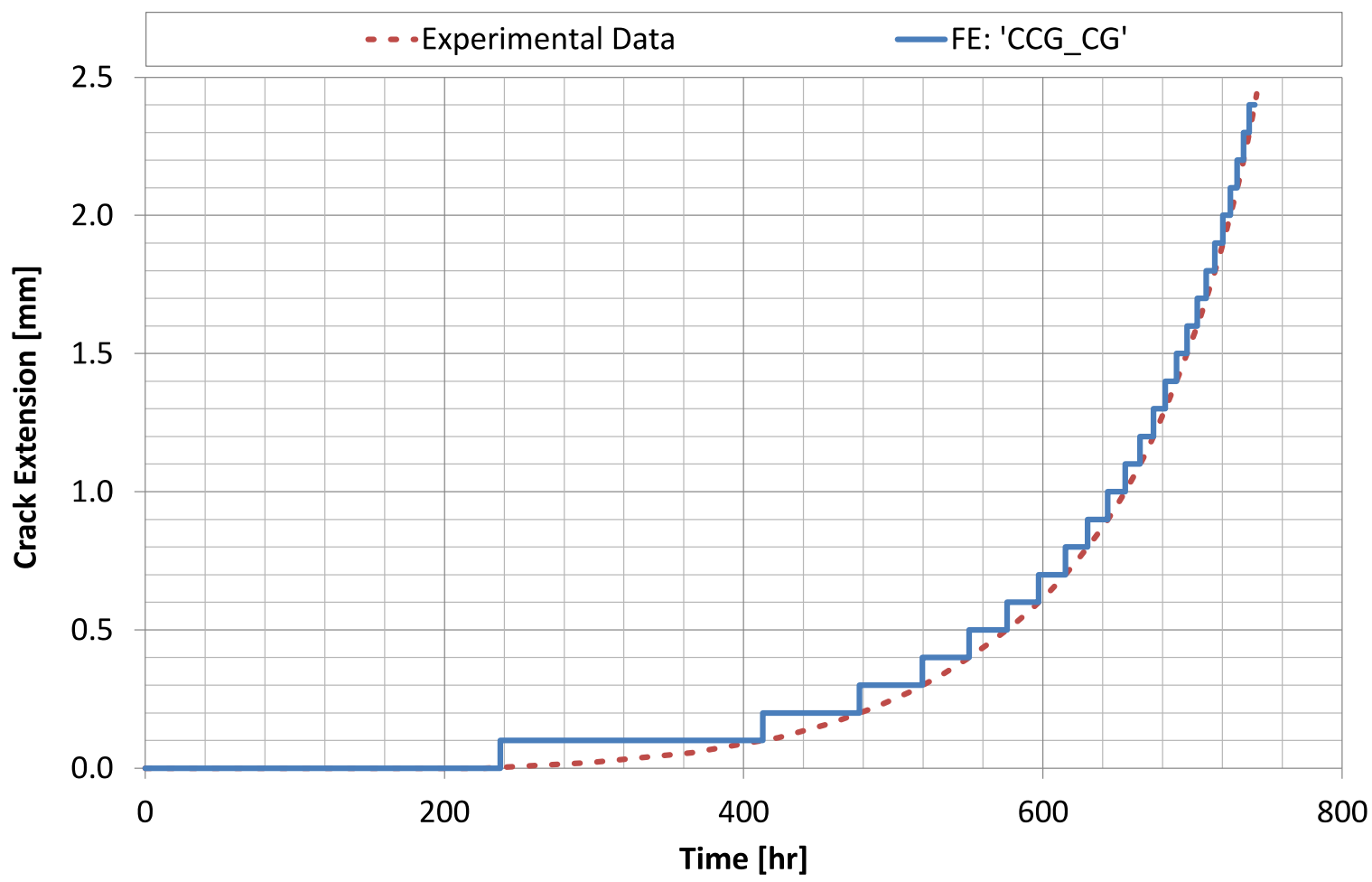

Figure 24: Comparison of experimental crack growth with the incremental crack growth applied to FE model 'CCG_CG'. 


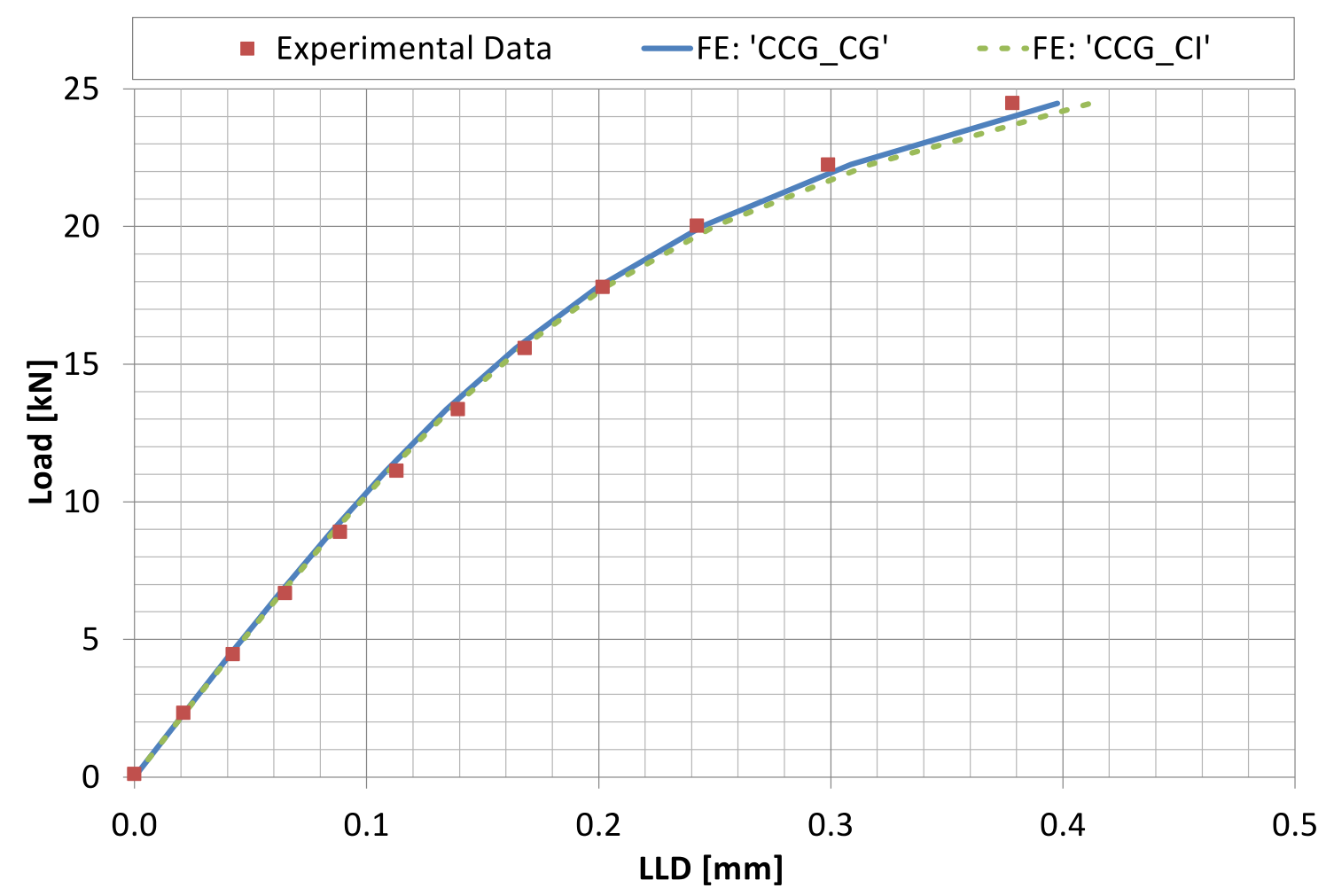

Figure 25: The structural response during load-up observed experimentally and predicted from the FE models.

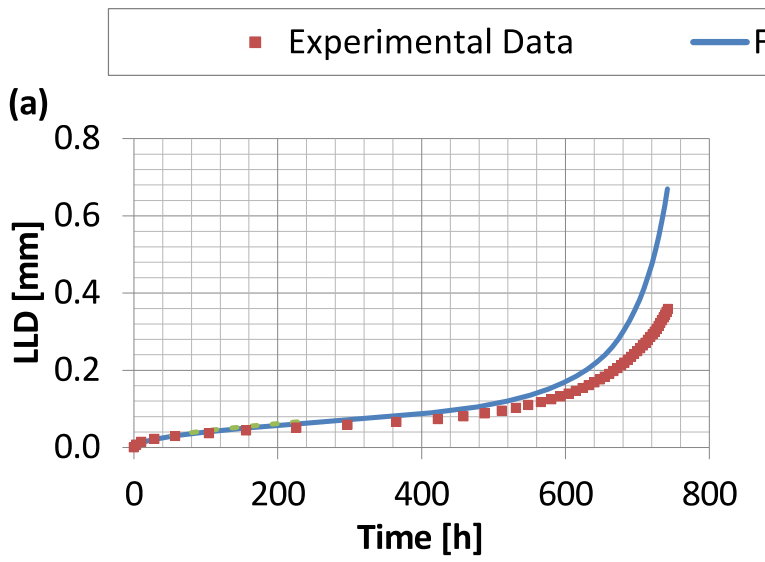
FE: 'CCG_CG' ‥FE: 'CCG_Cl'

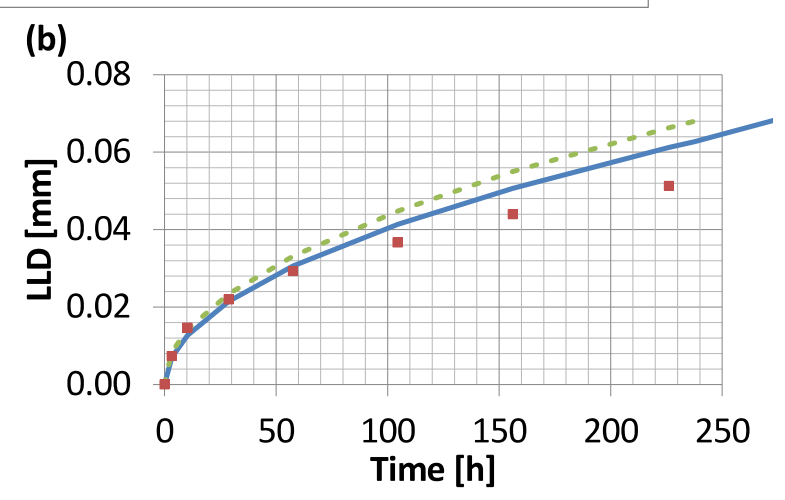

Figure 26: The time-dependent LLD response observed experimentally and predicted from the FE models showing (a) the entire test, and (b) the initial 250 hours. 

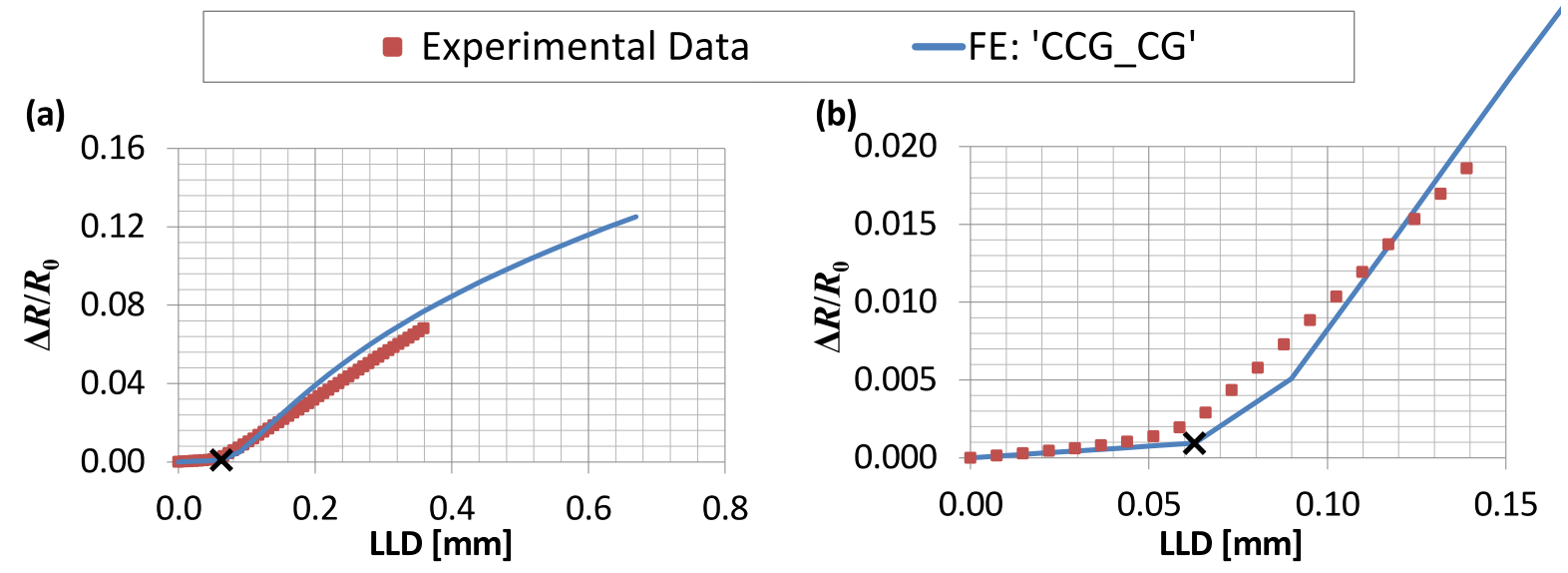

Figure 27: Relationship between the relative change in resistance and LLD observed experimentally and predicted from FE model 'CCG_CG' showing (a) the entire test, and (b) the initial part of the test. The onset of crack growth in the FE model is identified by the ' $X$ '.

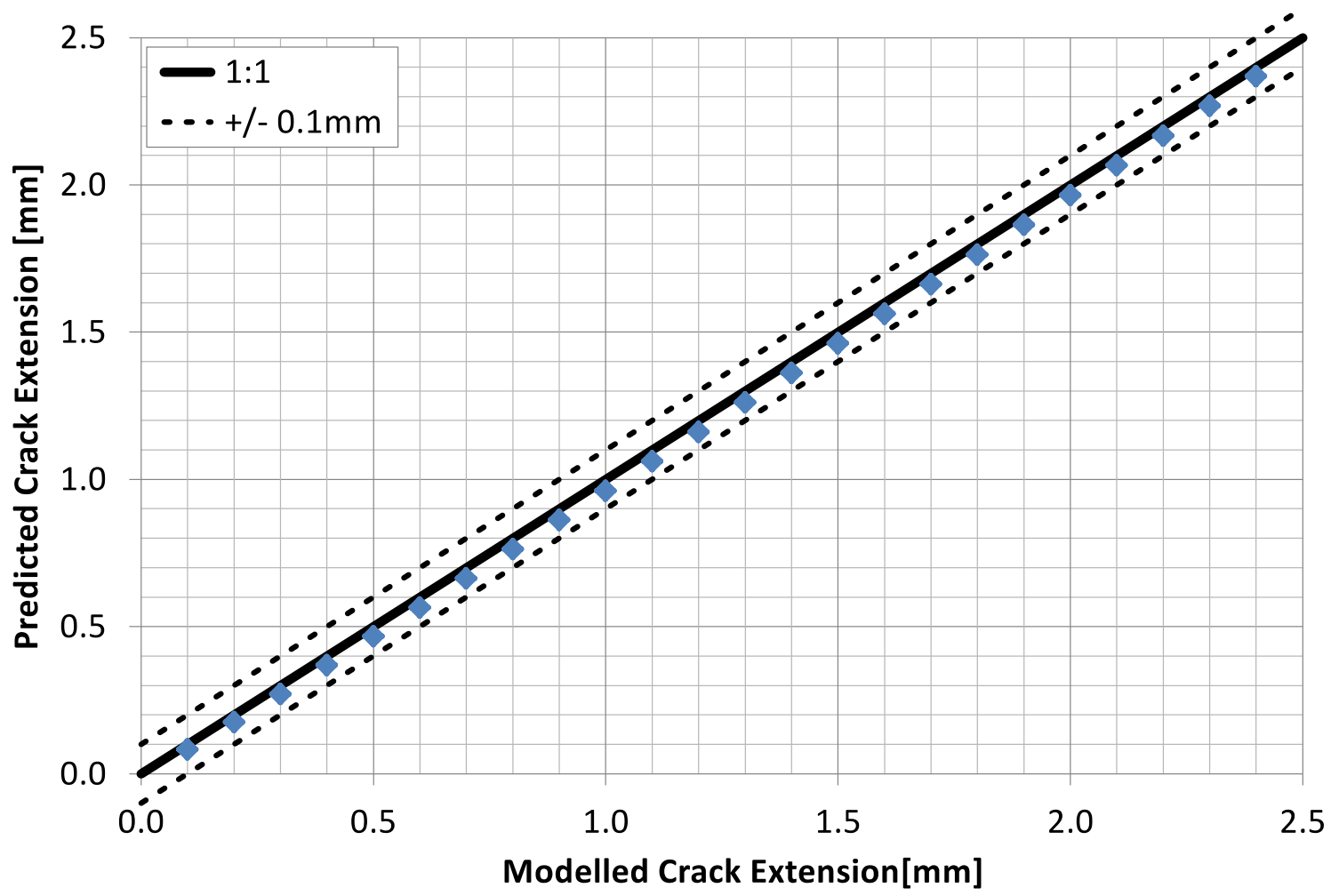

Figure 28: Comparison of the modelled crack extension and the crack extension predicted from the PD values obtained from the FE analysis. 
(a)

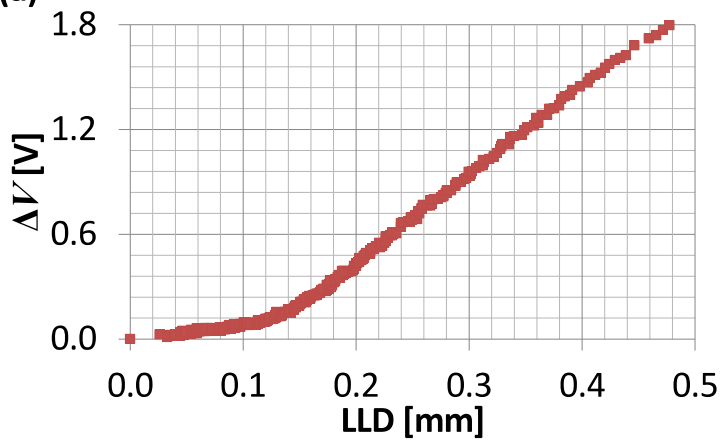

(b)

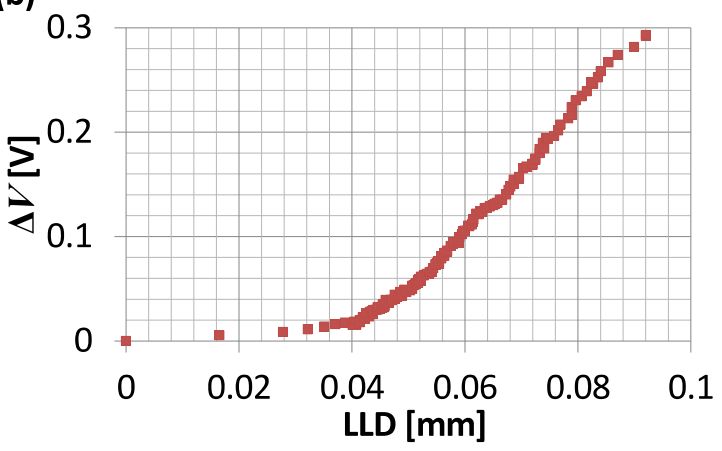

Figure 29: Relationship between PD and LLD during the initial part of a CCG test performed on (a) 1/2Cr1/4Mo1/4V (Specimen No: 30334_6), and (b) Esshete Stainless Steel (Specimen No: 2).

(a)

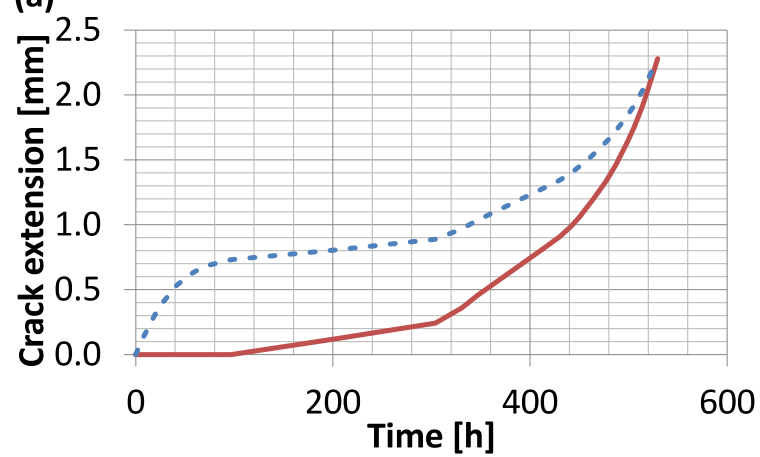

-..ASTM Method

(b)

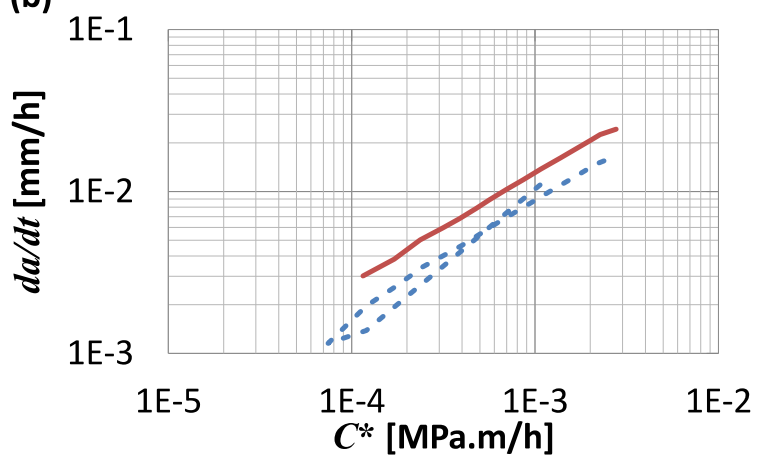

Figure 30: Comparison of the two methods of analysing PD data from CCG tests, based on test ID 1, showing (a) predicted crack extension with time, and (b) correlation between crack growth rate and $C^{*}$. 


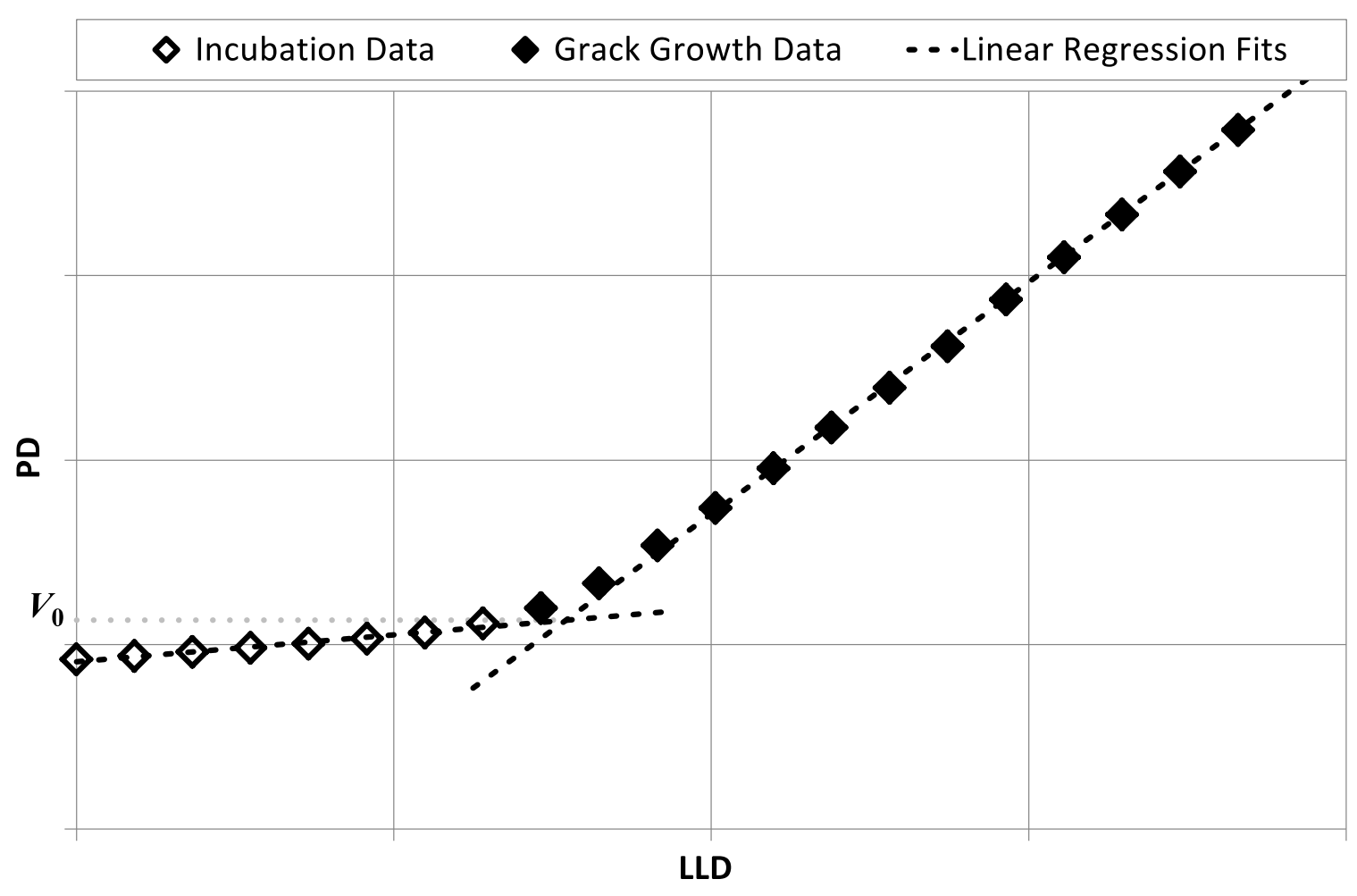

Figure 31: Schematic representation of the proposed method of interpreting PD data obtained from a CCG test performed on creep ductile material. 